This article has been accepted for publication in Monthly Notices of the Royal Astronomical Society (C): 2016 The Authors. Published by Oxford University Press on behalf of the Royal Astronomical Society. All rights reserved. 


\title{
LITTLE THINGS in 3D: robust determination of the circular velocity of dwarf irregular galaxies
}

\author{
G. Iorio, ${ }^{1,2 \star}$ F. Fraternali, ${ }^{1,3}$ C. Nipoti, ${ }^{1}$ E. Di Teodoro, ${ }^{4}$ J. I. $\operatorname{Read}^{5}$ and G. Battaglia ${ }^{6,7}$ \\ ${ }^{1}$ Dipartimento di Fisica e Astronomia, Università di Bologna, Viale Berti Pichat 6/2, I-40127 Bologna, Italy \\ ${ }^{2}$ INAF - Osservatorio Astronomico di Bologna, via Ranzani 1, I-40127 Bologna, Italy \\ ${ }^{3}$ Kapteyn Astronomical Institute, University of Groningen, Postbus 800, NL-9700 AV Groningen, the Netherlands \\ ${ }^{4}$ Research School of Astronomy and Astrophysics - The Australian National University, Canberra, ACT 2611, Australia \\ ${ }^{5}$ Department of Physics, University of Surrey, Guildford GU2 7XH Surrey, UK \\ ${ }^{6}$ Instituto de Astrofisica de Canarias, calle Via Lactea s/n, E-38205 La Laguna, Tenerife, Spain \\ ${ }^{7}$ Dpto. Astrofisica, Universidad de La Laguna, E-38206 La Laguna, Tenerife, Spain
}

Accepted 2016 December 14. Received 2016 November 11; in original form 2016 September 17

\begin{abstract}
Dwarf irregular galaxies (dIrrs) are the smallest stellar systems with extended $\mathrm{H}$ I discs. The study of the kinematics of such discs is a powerful tool to estimate the total matter distribution at these very small scales. In this work, we study the Hi kinematics of 17 galaxies extracted from the 'Local Irregulars That Trace Luminosity Extremes, The H I Nearby Galaxy Survey' (LITTLE THINGS). Our approach differs significantly from previous studies in that we directly fit 3D models (two spatial dimensions plus one spectral dimension) using the software

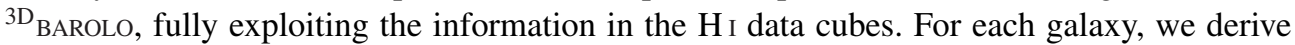
the geometric parameters of the $\mathrm{H}$ I disc (inclination and position angle), the radial distribution of the surface density, the velocity-dispersion $\left(\sigma_{v}\right)$ profile and the rotation curve. The circular velocity $\left(V_{\mathrm{c}}\right)$, which traces directly the galactic potential, is then obtained by correcting the rotation curve for the asymmetric drift. As an initial application, we show that these dIrrs lie on a baryonic Tully-Fisher relation in excellent agreement with that seen on larger scales. The final products of this work are high-quality, ready-to-use kinematic data $\left(V_{\mathrm{c}}\right.$ and $\left.\sigma_{v}\right)$ that we make publicly available. These can be used to perform dynamical studies and improve our understanding of these low-mass galaxies.
\end{abstract}

Key words: galaxies: dwarf-galaxies: ISM-galaxies: kinematics and dynamics-galaxies: structure.

\section{INTRODUCTION}

The H $21 \mathrm{~cm}$ emission line is a powerful tool for studying the dynamics of late-type galaxies since it is typically detected well beyond the optical disc and is not affected by dust extinction. Decades ago, the rise of the radio-interferometers allowed many authors to obtain high-resolution rotation curves for large samples of spiral galaxies (e.g. Bosma 1978, 1981; Bosma, Goss \& Allen 1981; van Albada et al. 1985; Begeman 1987). These studies found that the gas rotation remains nearly flat also in the outermost disc, where the visible matter fades and one would expect a nearly Keplerian fall-off of the rotation curve. The flat rotation curves of spirals are one of the most robust indications for the existence of dark matter (DM); gas-rich late-type galaxies are an ideal laboratory for studying the properties of DM haloes. In this context, dwarf irregular galaxies (dIrrs) are also studied with great interest. Unlike large spirals, they appear to be dominated by DM down to their very central regions.

^E-mail: giuliano.iorio@unibo.it
Thus, the determination of the DM density distribution is nearly independent of the mass-to-light ratio of the stellar disc (e.g. Casertano \& van Gorkom 1991; Côté, Carignan \& Freeman 2000).

The standard method to extract a galaxy rotation curve is based on the fitting of a tilted-ring model to a $2 \mathrm{D}$ velocity field (e.g. Begeman 1987; Schoenmakers, Franx \& de Zeeuw 1997). The velocity field is extracted from the $\mathrm{H}$ I data cube by deriving the representative velocity of the line profile at each spatial pixel. There are different methods to define a representative velocity, for instance using the intensity-weighted mean (e.g. Rogstad \& Shostak 1971), or by fitting functional forms (e.g. single Gaussian, Begeman 1987 or multiple Gaussians, Oh et al. 2015, hereafter O15). The 2D approach has been used in several numerical algorithms such as ROTCUR (Begeman 1987), RESWRI (Schoenmakers et al. 1997), KINEMETRY (Krajnović et al. 2006) and DISKFIT (Spekkens \& Sellwood 2007). All of these codes have been very useful to improve our understanding of the kinematics of late-type galaxies. However, working in 2D has a drawback: the results depend on the assumptions made when extracting the velocity field and are also 
affected by the instrumental resolution (the so-called beam smearing; Bosma 1978; Begeman 1987). These problems are especially relevant in the study of the kinematics of dIrrs since the $\mathrm{HI}_{\mathrm{I}}$ line profiles can be heavily distorted by both non-circular motions and noise. As a consequence, the extraction of the velocity field can be challenging (see e.g. Oh et al. 2008). Furthermore, dIrrs are often observed with a limited number of resolution elements because of their limited extension on the sky. The effect of beam smearing can also make the estimate of both the rotation curve and the inclination of the disc uncertain (Begeman 1987).

The pioneering work of Swaters (1999) showed that these problems can be solved with an alternative approach: the properties of the $\mathrm{H}_{\mathrm{I}}$ disc can be retrieved with a direct modelling of the 3D data cube (two spatial axes plus one spectral axis) without explicitly extracting velocity fields. In practice, a $3 \mathrm{D}$ method consists of a data-model comparison of $n_{\mathrm{ch}}$ maps (where $n_{\mathrm{ch}}$ is the number of spectral channels) instead of the single map represented by the velocity field. The best advantage of this approach is that the data cube models are convolved with the instrumental response, so the final results are not affected by the beam smearing. Swaters (1999), Gentile et al. (2004), Lelli, Fraternali \& Sancisi (2010) and Lelli, Verheijen \& Fraternali (2014b) used a 3D visual comparison between data cubes and model cubes to correct and improve the results obtained with the classical 2D methods. However, a by-eye inspection of the data is time intensive and subjective. Modern software like TIRIFIC

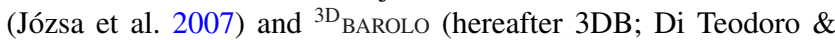
Fraternali 2015) can perform a full 3D numerical minimization on the whole data cube.

In this paper, we exploit the power of the 3D approach by applying 3DB to a sample of 17 dIrrs taken from the Local Irregulars That Trace Luminosity Extremes, The H I Nearby Galaxy Survey (LITTLE THINGS; hereafter LT) sample (Hunter et al. 2012). 3DB has been extensively tested on mock $\mathrm{H}_{\mathrm{I}}$ data cubes corresponding to low-mass isolated dIrrs. Read et al. (2016c) show that the rotation curve is well recovered even for a starbursting dwarf, provided that the inclination of the $\mathrm{HI}$ disc is higher than $40^{\circ}$.

The final products of our analysis are ready-to-use rotation curves corrected for the instrumental effects (see Section 3.2) and for the asymmetric drift (see Section 4.3.1). The rotation curves can be used to study the dynamics of dIrrs (e.g. van Eymeren et al. 2009b; Read et al. 2016a,c) or to extend the study of the scaling relations on small scales (e.g. Brook, Santos-Santos \& Stinson 2016; Lelli, McGaugh \& Schombert 2016). An unbiased estimate of the rotation curves of dIrrs is also essential to shed light on the well-known cosmological tensions on small scale: the long debated 'cusp-core problem' (de Blok \& Bosma 2002; Oh et al. 2011; Read, Agertz \& Collins 2016b), the 'missing-satellites problem' (Klypin et al. 1999; Read et al. 2016a) and the 'toobig-to-fail problem' (Read et al. 2006; Boylan-Kolchin, Bullock \& Kaplinghat 2011; Read et al. 2016a). These 'problems' are discrepancies between the properties of DM haloes predicted by cosmological simulations and those inferred from observations. In this context, it is therefore crucial to obtain reliable estimates of the DM distribution in real galaxies.

In this work, we focus in particular on the asymmetric-drift correction (see Section 4.3.1). This correction term is usually applied without considering the errors made in its calculation, but these can be very large and should be taken into account. The propagation of the asymmetric-drift errors has a great impact on the determination of the circular velocity of galaxies in which the contribution of the gas velocity dispersion is important (see for example DDO 210 in Section 5). We developed a method to calculate and propagate the asymmetric-drift uncertainties: as a result, the quoted errors on the final corrected rotation curves are a robust description of the real uncertainties.

The paper is organized as follows. In Section 2, we illustrate the sample and the data used in this work. In Section 3, we briefly introduce the tilted-ring model and 3DB. In Section 4, we describe in detail the analysis applied to our data. Section 5 shows the results of our analysis for each galaxy in our sample. In Section 6, we look at whether the low-mass dIrrs that we study here lie on the baryonic Tully-Fisher relation (BTFR). In Section 7, we discuss the assumptions made to derive the rotation curves and we compare our results with previous work. A summary is given in Section 8 .

\section{SAMPLE AND DATA}

\subsection{Sample selection}

The galaxies used in this work represent a sub-sample of the galaxies of the LT survey (Hunter et al. 2012), which is a sample of 37 dIrrs and 4 blue compact dwarfs (BCDs) located in the Local Volume within $11 \mathrm{Mpc}$ from the Milky Way. LT combines archival and new H I observations taken with the very large array to obtain a very high spatial and spectral resolution data set (see Table 1). The objects in the LT sample have been chosen to be both isolated and representative of the full range of dIrr properties (Hunter et al. 2012).

We built our sample selecting dIrrs that are well suited to study the circular motion of the $\mathrm{H}$ I discs. To this end, we excluded from our selection all the objects seen at low inclination angles and the four BCDs. The inclination angle $i$ is defined as the angle between the plane of the disc and the line of sight such that $i=90^{\circ}$ for an edge-on galaxy and $i=0^{\circ}$ for a face-on galaxy. The estimate of $i$ in nearly face-on galaxies $\left(i<40^{\circ}\right)$ is extremely difficult both from the kinematic fit (Begeman 1987) and from the analysis of the $\mathrm{H}$ map (Read et al. 2016c). Moreover, for $i<40^{\circ}$, relatively small errors on $i$ have a great impact on the deprojection of the observed rotational velocity (see equation 1). As a consequence, the final rotation curves of these nearly face-on galaxies could be biased and unreliable with both 2D (Oman et al. 2016) and 3D methods (Read et al. 2016c). Among the objects with $i>40^{\circ}$, we selected 17 dIrrs, about half of the original LT sample.

We checked, using a Kolmogorov-Smirnov (KS) test, that our sub-sample preserves the statistical distribution of the galactic properties such as distance (KS $p$-value 0.96 ), absolute magnitude (KS $p$-value 0.99), star formation rate density (SFRD, KS $p$-value 0.92 ) and baryonic mass (KS $p$-value 0.99). Because of our rejection criterion, the average $i$ in our sub-sample $\left(58^{\circ}\right)$ is higher than the one measured in the LT sample $\left(50^{\circ}\right)$. Fig. 1 shows the distributions of the distances and of the absolute magnitudes of our sample and the original LT sample.

It is worth pointing out that our rejection criterion is based on $i$ estimated for the stellar disc from the $V$-band photometry (Hunter \& Elmegreen 2006). When we re-estimated $i$ from the H I data cubes (see Section 4.2), we found that four galaxies (DDO 47, DDO 50, DDO 53 and DDO 133) have an average $i$ lower than $40^{\circ}$ (see Table 2). We did not exclude these galaxies from our sample, but our results for these objects should be treated with caution. In particular, the formal errors on the velocities and on $i$ could underestimate the real uncertainties (see notes on the individual galaxies in Section 5).

In conclusion, our sample comprises 17 objects covering the stellar mass range $5 \times 10^{5} \lesssim \mathrm{M}_{*} / \mathrm{M}_{\odot} \lesssim 10^{8}$, with a mean SFRD of about $0.006 \mathrm{M}_{\odot} \mathrm{yr}^{-1} \mathrm{kpc}^{-2}$ and a mean specific SFRD (SSFRD) of about $2.26 \times 10^{-10} \mathrm{yr}^{-1} \mathrm{kpc}^{-2}$ (data from Hunter et al. 2012 
Table 1. Properties of the sample of galaxies studied in this work: (1) distance in Mpc (Hunter et al. 2012 and references therein); (2) V-band absolute magnitude (Hunter et al. 2012 and references therein); (3) conversion factor from arcsec to pc; (4) weighted scheme used to produce the final data cube: 'na' for natural 'ro' for robust. $\mathrm{A}^{\dagger}$ means that we have further smoothed the original data cube to the beam indicated in Column 5; (5) beam major $\times$ minor axis in arcsec of the data used for the kinematic fits (see Section 2.2); (6) channel separation in $\mathrm{km} \mathrm{s}^{-1}$ (this value indicates also the velocity resolution of the data cube in terms of FWHM); (7) total H I mass (see Section 4.1.1); (8) rms noise per channel estimated with 3DB; (9) 3 $\sigma$ pseudo-noise in the total map (see Section 4.1.2). Note that DDO 87 has a slightly different distance with respect to the one reported in Hunter et al. (2012) because their value is a typo (the correct value is given in Croxall et al. 2009).

\begin{tabular}{|c|c|c|c|c|c|c|c|c|c|}
\hline Galaxy & $\begin{array}{c}D \\
(\mathrm{Mpc}) \\
(1)\end{array}$ & $\begin{array}{c}M_{V} \\
(\mathrm{mag}) \\
(2)\end{array}$ & $\begin{array}{c}f_{\mathrm{c}} \\
\left(\mathrm{pc} \operatorname{arcsec}^{-1}\right) \\
(3)\end{array}$ & $\begin{array}{l}\text { Cube } \\
\text { (4) }\end{array}$ & $\begin{array}{c}\text { Beam } \\
(\operatorname{arcsec} \times \operatorname{arcsec}) \\
(5)\end{array}$ & $\begin{array}{c}\text { Ch. sep } \\
\left(\mathrm{km} \mathrm{s}^{-1}\right) \\
(6)\end{array}$ & $\begin{array}{c}M_{\mathrm{HI}} \\
\left(10^{7} \mathrm{M}_{\odot}\right) \\
(7)\end{array}$ & $\begin{array}{c}\sigma_{\mathrm{ch}} \\
\left(\mathrm{mJy} \mathrm{Beam}^{-1}\right) \\
(8)\end{array}$ & $\begin{array}{c}\sigma_{3 \mathrm{~T}} \\
\left(\mathrm{M}_{(9)} \mathrm{pc}^{-2}\right)\end{array}$ \\
\hline DDO 47 & 5.2 & -15.5 & 25.2 & na & $16.1 \times 15.3$ & 2.6 & $35.3 \pm 0.6$ & 0.61 & 0.41 \\
\hline DDO 50 & 3.4 & -16.6 & 16.5 & $\mathrm{na}^{\dagger}$ & $15.0 \times 15.0$ & 2.6 & $59.5 \pm 1.6$ & 0.82 & 0.65 \\
\hline DDO 52 & 10.3 & -15.4 & 49.9 & na & $11.8 \times 7.2$ & 2.6 & $22.3 \pm 0.1$ & 0.46 & 1.20 \\
\hline DDO 101 & 6.4 & -15.0 & 31.0 & ro & $8.3 \times 7.0$ & 2.6 & $1.8 \pm 0.2$ & 0.50 & 0.90 \\
\hline DDO 126 & 4.9 & -14.9 & 23.7 & na & $12.2 \times 9.3$ & 2.6 & $12.8 \pm 0.1$ & 0.41 & 1.27 \\
\hline DDO 133 & 3.5 & -14.8 & 17.0 & ro & $12.4 \times 10.8$ & 2.6 & $10.0 \pm 0.2$ & 0.35 & 0.89 \\
\hline DDO 154 & 3.7 & -14.2 & 18.0 & $\mathrm{na}^{\dagger}$ & $15.0 \times 15.0$ & 2.6 & $25.1 \pm 0.1$ & 0.44 & 0.52 \\
\hline DDO 168 & 4.3 & -15.7 & 20.8 & na & $15.0 \times 15.0$ & 2.6 & $25.1 \pm 0.1$ & 0.47 & 0.54 \\
\hline DDO 210 & 0.9 & -10.9 & 4.4 & na & $16.6 \times 14.1$ & 1.3 & $0.2 \pm 0.1$ & 0.75 & 0.59 \\
\hline WLM & 1.0 & -14.4 & 4.8 & $\mathrm{na}^{\dagger}$ & $25.0 \times 25.0$ & 2.6 & $5.6 \pm 0.1$ & 2.00 & 0.58 \\
\hline
\end{tabular}
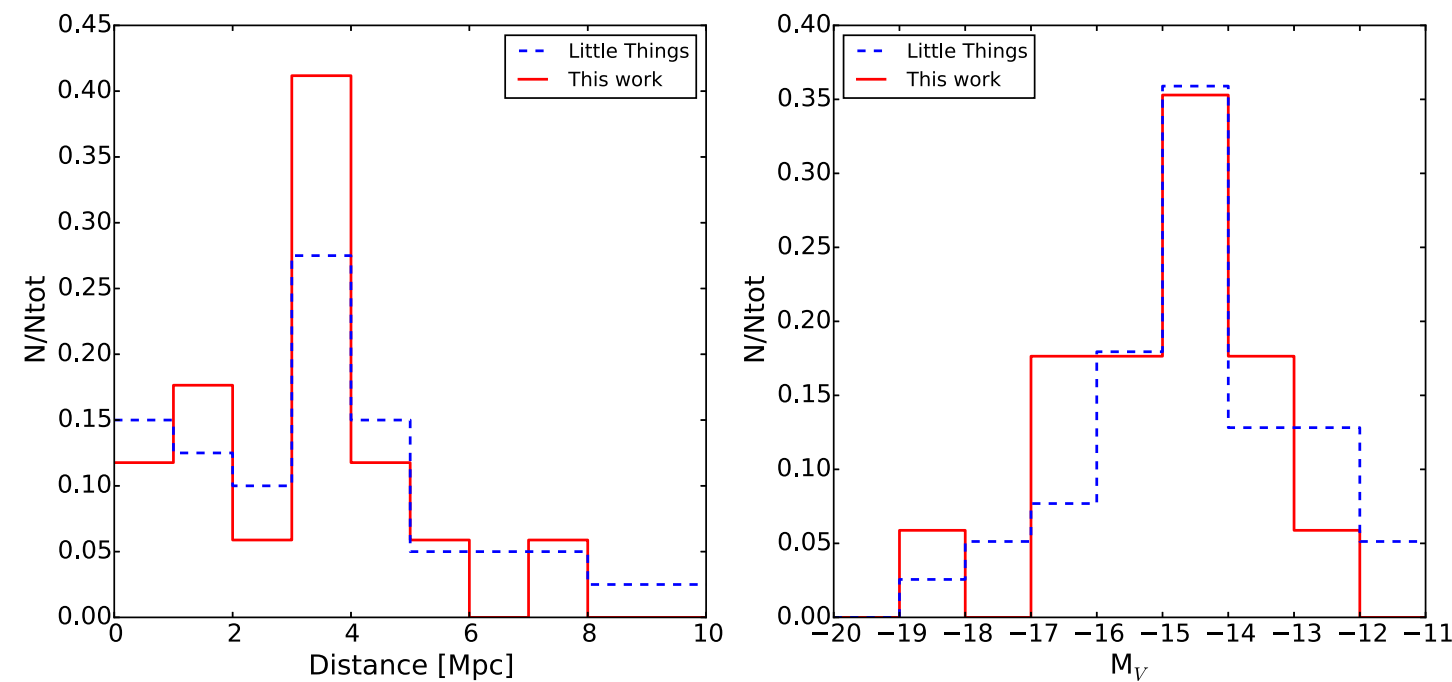

Figure 1. Distribution of the Galactocentric distances (left-hand panel) and of the absolute magnitudes (right-hand panel) for the sample of galaxies studied in this work (red histograms) and for the whole LT sample (blue dashed histograms). Data from Hunter et al. (2012) and references therein.

and references therein). For comparison the SFRD and the SSFRD of the complete LT sample are $0.007 \mathrm{M}_{\odot} \mathrm{yr}^{-1} \mathrm{kpc}^{-2}$ and $2.95 \times$ $10^{-10} \mathrm{yr}^{-1} \mathrm{kpc}^{-2}$, respectively (Hunter et al. 2012 and references therein).

\subsection{The data}

The $\mathrm{H}_{\text {I }}$ data cubes were taken from the publicly available archive of the LT survey. ${ }^{1}$ For each galaxy, LT provides two data cubes

\footnotetext{
${ }^{1}$ https://science.nrao.edu/science/surveys/littlethings
}

that differ in the weight scheme used to reduce the raw data: the natural data cube offers the best signal-to-noise ratio, the robust data cube gives the best angular resolution. We preferred the natural data cubes for two main reasons: (i) a high signal-to-noise ratio allows us to have enough sensitivity to extend the study of the kinematics to large radii; (ii) 3D methods are not dramatically influenced by the resolution of the data (see Section 3). In some cases (DDO 50, DDO 154, NGC 1569, NGC 2366, UGC 8508 and WLM), the galaxies are so extended in the sky that we further smoothed the data cube maintaining a good spatial resolution. Instead, we have chosen the robust data cube when the natural data cube gives too few resolution elements to sample the H I disc (five cases, see Table 1). The mean 
Table 2. Fit assumptions: (1) outermost radius in arcsec (see Section 4.1.2); (2) outermost radius in kpc (see Section 4.1.2); (3) sampling radius used in the data cube fitting; (4a and 4b) assumed galactic centre in RA (hh mm ss.s) and Dec. (dd mm ss) coordinate system (J2000); (5) assumed systemic velocity (see Section 3.2.1); (6) initial guesses for $i$; (7) initial guesses for PA. ${ }^{S}$ indicates that the values is estimated from the stellar disc (Hunter \& Elmegreen 2006), ${ }^{B}$ using $3 \mathrm{DB},{ }^{H}$ using the fit of the contours of the $\mathrm{H}$ I total map (see Appendix A for details). ${ }^{T}$ indicates peculiar cases (see the individual galactic description for details). Results: (8) mean circular velocity of the outer disc (see Section 6); (9) median of the best-fitting velocity dispersion found with 3DB; (10) median of the best-fitting PA found with 3DB; (11) median of the best-fitting $i$ found with 3DB. ${ }^{\dagger}$ indicates nearly face-on galaxies in which the quoted errors on $<i>$ and $V_{\mathrm{o}}$ could underestimate the real uncertainties.

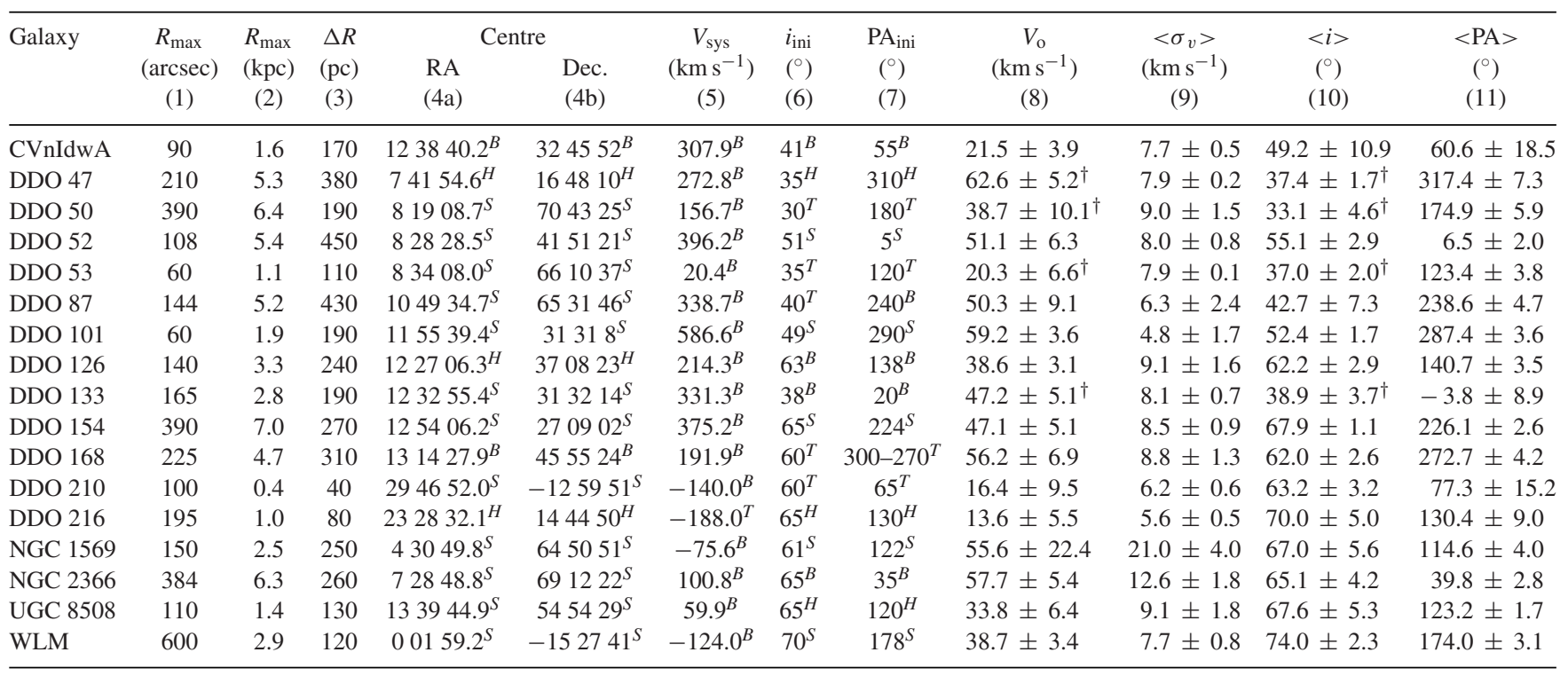

spatial resolution is about 240 pc ranging from about 60 (DDO 210) to about $460 \mathrm{pc}$ (DDO 87). These data are summarized in Columns 4-6 of Table 1. The rotation curves of all the galaxies in our sample have been already derived with the classical 2D approach in 015 . The masses and the surface-density profiles of the stellar discs for all the galaxies can be found in Zhang et al. (2012), except for DDO 47 for which they can be found in Makarova (1999) and in Walter \& Brinks (2001).

\section{THE METHOD}

\subsection{Tilted-ring model}

Since Rogstad, Lockhart \& Wright (1974), the most common approach to study the kinematics of the HI discs is the so-called 'tilted-ring model'. In practice, the rotating disc is broken into a series of independent circular rings with radius $R$, each with its kinematic and geometric properties. The projection of each ring on the sky is an elliptical ring with semimajor axis $R$ and semiminor axis $R \cos i$. Throughout the paper, $R$ indicates both the radius of the circular ring and the semimajor axis of its projection. For a given projected ring, the projected velocity along the line of sight $\left(V_{\text {los }}\right)$ is

$V_{\mathrm{los}}=V_{\mathrm{sys}}+V_{\mathrm{rot}}(R) \cos \theta \sin i$,

where $V_{\text {sys }}$ is the systemic velocity of the galaxy, $V_{\text {rot }}$ is the rotation velocity of the gas at radius $R, \theta$ is the azimuthal angle of the rings in the plane of the galaxy (related to $i$, to the galaxy centre and to the position angle; equations $2 \mathrm{a}$ and $2 \mathrm{~b}$ in Begeman 1987). The position angle (PA, hereafter) is defined as the angle between the north direction on the sky and the projected major axis of the receding half of the rings.

It is worth noting that equation (1) is strictly valid only assuming that the gas is settled in a razor-thin disc and it describes only the circular motion of the gas. Deviations from pure circular orbits can include radial motions due to inflow-outflow, non-circular motions due to deviation from symmetry of the galactic potential (spiral arms, mergers, misaligned DM halo and so on; see e.g. Schoenmakers et al. 1997; Swaters et al. 1999) and small-scale perturbations due to the star formation activity (stellar winds, supernovae, see e.g. Read et al. 2016c). The tilted-ring model allows us to trace the radial variation of the $\mathrm{H}$ I disc geometry and model the so-called 'warp' (García-Ruiz, Sancisi \& Kuijken 2002; Battaglia et al. 2006). Moreover, using the ring decomposition it is possible also to obtain radial profiles from the integrated 2D maps (e.g. the H I surface density, see Section 4.1).

In this work, we measure the kinematic and geometric properties of the galaxies in our sample with the publicly available software $3 \mathrm{DB}^{2}$ (Di Teodoro \& Fraternali 2015) that is a 3D code based on the tilted-ring approach.

\section{2 ${ }^{3 \mathrm{D}}$ BAROLO}

$3 \mathrm{DB}$ is a $3 \mathrm{D}$ method that performs a tilted-ring analysis on the whole data cube. In practice, for each sampling radius it builds a ring model based on equation (1), then it calculates the residuals between the emission of the model and of the data pixel-by-pixel along the ring in the data cube. The final parameters of each ring are found through the minimization of these residuals. Before the comparison between the data cube and the model, the latter is smoothed to the spatial and spectral instrumental resolution. This ensures full control of the observational effects and in particular a proper account of beam smearing that can strongly affect the derivation of the rotation velocities in the inner regions of dwarf galaxies (see e.g. Swaters 1999). Moreover 3DB fits, at the same time, the rotation

\footnotetext{
${ }^{2}$ http://editeodoro.github.io/Bbarolo/
} 
velocity and the velocity dispersion instead of treating them as separate components as done in the classical 2D approach (e.g. Tamburro et al. 2009; O15).

In conclusion, 3DB fits up to eight parameters for each ring in which the galaxy is decomposed: central coordinates, $V_{\text {sys }}, i, \mathrm{PA}$, $\mathrm{H}_{\mathrm{I}}$ surface density $(\Sigma), \mathrm{H}_{\mathrm{I}}$ thickness $\left(z_{\mathrm{d}}\right), V_{\text {rot }}$ and the velocity dispersion $\left(\sigma_{v}\right)$. 3DB separates the genuine $\mathrm{H}_{\mathrm{I}}$ emission from the noise by building a mask: only the pixels containing a signal above a certain threshold from the noise are taken into account. The noise estimated with 3DB for each galaxy is reported in the Column 8 of Table 1. Further details on 3DB can be found in Di Teodoro \& Fraternali (2015).

3DB works well both on high-resolution and low-resolution data (Di Teodoro \& Fraternali 2015). This allows us to use the optimal compromise between spatial resolution and sensitivity, as already discussed in Section 2.2. Read et al. (2016c) showed that 3DB is capable of obtaining a good estimate of the kinematic parameters of mock $\mathrm{H}$ I data cubes corresponding to low-mass isolated dIrrs, similar to those that we will study here.

\subsubsection{Assumptions}

As the focus of this work is the kinematics $\left(V_{\text {rot }}\right.$ and $\left.\sigma_{v}\right)$ of the $\mathrm{HI}$ discs, we can consider the other six variables fitted by 3DB as "nuisance parameters'. We decided to reduce the relatively high number of parameters making assumptions on the $\mathrm{H}_{\mathrm{I}}$ surface density, $\mathrm{H}_{\mathrm{I}}$ scaleheight, systemic velocity and the position of the galactic centre.

(i) H I surface density: We remove the surface density from the list of the free parameters by normalizing the H I flux. 3DB implements two different normalization techniques: pixel-by-pixel or azimuthally averaged. In the first case the model is normalized locally to the value of the total $\mathrm{H}_{\mathrm{I}}$ map, while in the second case the model is normalized to the azimuthally averaged flux in each ring. For a full description of these normalization techniques, see the 3DB reference paper (Di Teodoro \& Fraternali 2015). We decided to use the local pixel-by-pixel option, because this approach is convenient to take into account the asymmetry of the $\mathrm{H}$ I distribution and to avoid that regions with peculiar emission (e.g. clumps or holes) affect excessively the global fit (see Lelli et al. 2012a,b). However, we checked that the results obtained with the two normalizations are fully compatible within the errors.

(ii) $H_{\text {I }}$ scaleheight: $3 \mathrm{DB}$ includes the possibility to fit the $\mathrm{H}_{\mathrm{I}}$ scaleheight. This is something exclusive to 3D methods since there is no information about the scaleheight in the integrated 2D velocity field. However, the assumption of a thick disc is somewhat inconsistent with the 'tilted-ring model' (see Section 3.1): in the presence of a thick disc along the line of sight we are accumulating emission form different rings, hence a ring-by-ring analysis cannot incorporate this effect. To be fully consistent, we should set the value of the scaleheight $z_{\mathrm{d}}$ to 0 ; however, it turns out that, assuming $z_{d}=0$ the galactic models made with 3DB have a too sharp cut-off at the border of rings. Therefore, we decided to set for all the galaxies a scaleheight of $100 \mathrm{pc}$, constant in radius. In Section 7.1, we discuss in detail the effect of this assumption.

(iii) Systemic velocity: We fix the systemic velocity for all rings to the value calculated as

$V_{\text {sys }}=0.5\left(V 20_{\text {app }}+V 20_{\text {rec }}\right)$,

where $V 20$ is the velocity where the flux of the global $\mathrm{H}$ I profile is 20 per cent of the flux peak, while 'app' and 'rec' indicate the approaching and the receding halves of the galaxy. (iv) Centre of the galaxy: We fix the position of the galactic centre for all rings to the value found with one of the methods described in Appendix A.

\section{DATA ANALYSIS}

\subsection{H I total map}

Before starting the kinematic fit procedure, for each galaxy we produce $\mathrm{H}_{\mathrm{I}}$ total maps from the data cubes. This step is needed both to have an initial rough estimate of the geometrical properties of the $\mathrm{H}$ I discs and to define the maximum radius to use in the kinematic fit. Moreover, we use it to extract the H I surface-density profiles using the best-fitting parameters of the disc (Section 4.2). The H I surface-density profile is crucial to correct the estimated rotation curve for the asymmetric drift (Section 4.3.1).

\subsubsection{H I spatial distribution}

Integrating the signal of each pixel along the spectral axis, one obtains a 2D total map that represents the spatial distribution (on the sky) of the Hi emission. To build these maps, we masked the original data cube with the following procedure: first, we smoothed the original data to obtain a low-resolution cube (between two and three times the original beam, depending on the galaxy); then, a pixel is included in the mask only if its emission is above a certain threshold ( 2.5 of the noise $\sigma_{\mathrm{ch}}$ of the smoothed cube) in at least three adjacent channels. Finally we sum, along the spectral axis, all the flux of the pixel included in the mask obtaining a 2D map. This method produces a very clean final map with only a small contribution from the noise.

Following Roberts (1975), we can directly relate the intensity of the radio emission $(S)$ to the projected surface-density of the gas $\left(\Sigma_{\text {obs }}\right)$ as

$\frac{\Sigma_{\mathrm{obs}}(x, y)}{\mathrm{M}_{\odot} / \mathrm{pc}^{2}}=8794\left(\frac{S(x, y)}{\mathrm{Jy} \mathrm{Beam}^{-1}}\right)\left(\frac{\Delta V}{\mathrm{~km} \mathrm{~s}^{-1}}\right)\left(\frac{B_{\mathrm{maj}} B_{\mathrm{min}}}{\operatorname{arcsec}^{2}}\right)^{-1}$,

where $B_{\text {maj }}$ and $B_{\min }$ are the full width at half-maximum (FWHM) of the major and minor axes of the beam and $\Delta V$ is the channel separation of the data cube. We obtained the radial profile of $\Sigma_{\text {obs }}$ averaging the total map along elliptical rings defined by the disc geometrical parameters (see Section 4.2). The errors on $\Sigma_{\text {obs }}$ are calculated as the standard deviation of the averaged values. ${ }^{3}$ The intrinsic surface density $\left(\Sigma_{\text {int }}\right)$ is defined as the density of the $\mathrm{H}_{\mathrm{I}}$ disc integrated along the vertical axis of the galaxy. Assuming a razor-thin $\mathrm{H}_{\mathrm{I}}$ disc, the relation between $\Sigma_{\mathrm{obs}}$ and $\Sigma_{\text {int }}$ is simply

$\Sigma_{\text {int }}(\mathrm{R})=\Sigma_{\text {obs }}(\mathrm{R}) \cos i$,

because the area of the projected ring is smaller than the one of the intrinsic ring by a factor $\cos i$. Finally, we can have a measure of the total mass of the $\mathrm{H}$ I disc summing the observed surface densities

\footnotetext{
${ }^{3}$ Pixels in data cubes are correlated by the convolution with the instrumental beam occurring during the observations. As a consequence, the estimated errors are an underestimate of the real uncertainties (see Sicking 1997). However, the estimated errors for the surface density are already very large and we did not correct them. Moreover, the elliptical rings are usually much larger than the beam, so the correlation of the data is not expected to affect significantly the final estimate of the average and the error of the surface density.
} 


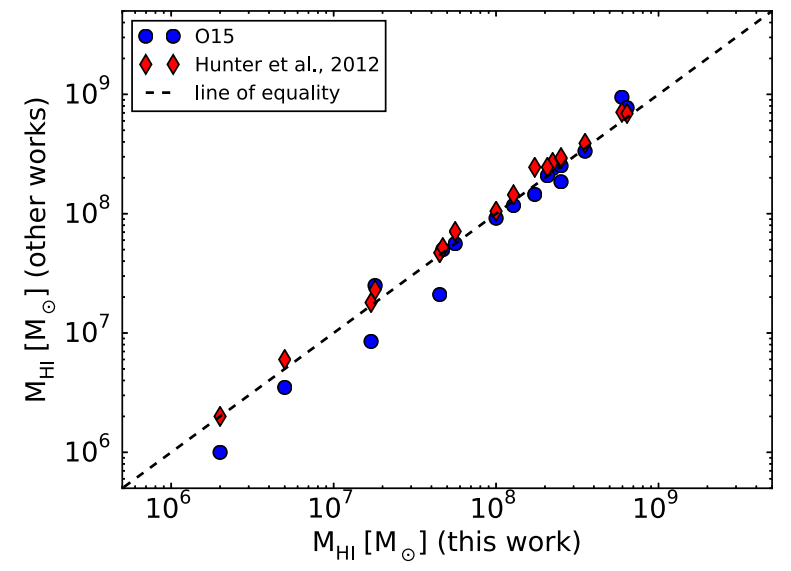

Figure 2. Comparison of the $\mathrm{H}_{\mathrm{I}}$ mass estimated in this work and in other works [O15, blue circles; Hunter et al. (2012), red diamonds].

of all pixels in the total map multiplied by the physical area of the pixels

$\frac{\mathrm{M}_{\mathrm{H}_{\mathrm{I}}}}{\mathrm{M}_{\odot}}=23.5\left(\frac{\delta}{\operatorname{arcsec}}\right)^{2}\left(\frac{D}{\mathrm{Mpc}}\right)^{2} \sum_{\text {pixels }}\left(\frac{\Sigma_{\mathrm{obs}}(x, y)}{\mathrm{M}_{\odot} \mathrm{pc}^{-2}}\right)$,

where $\delta$ is the size of the pixel and $D$ is the distance of the galaxy. To have an estimate of the uncertainties in this measure, we have built two other maps: a low-resolution map using the smoothed cube that we used to build the mask and an extremely low-resolution map smoothing again the cube to a beam of 40-60 arcsec. We applied the primary beam correction to the total H I map using the task PBCORR of GIPSY (van der Hulst et al. 1992). For each map, the mass is estimated using equation (5) and the method described above. In particular, we take as measure of the disc mass the average and the associated error the standard deviation of the values obtained in the three maps. The final estimates of $\mathrm{M}_{\mathrm{H}_{\mathrm{I}}}$ are listed in Table 1 (Column 7). The intrinsic surface-density profile and the H I map contours are shown respectively in the Box B (bottom right panel) and in the Box C (left-hand panel) of the summary plot of each galaxy (Section 5).

Fig. 2 shows the comparison between the mass estimated with our method and those estimated by $\mathrm{O} 15$ and Hunter et al. (2012). The relative difference between our measures and those of $\mathrm{O} 15$ is on average 20 per cent, with the largest discrepancies (up to 50 per cent) found at the low-mass end; these differences are mainly due to the different method used to estimate the total mass. The method used in Hunter et al. (2012) is similar to the one used in this work (Hunter et al. 2012 used only robust data cubes while we used both robust and natural data cubes; see Column 4 in Table 1): in this case the average relative difference is about 10 per cent. Because of the datareduction methods used in Hunter et al. (2012), the natural data cubes have systematically lower flux with respect to the robust ones (see Hunter et al. 2012 for the details), therefore our values are slightly lower.

\subsubsection{Noise in the total map and maximum radius}

In order to define a maximum radius for the $\mathrm{H}$ I disc, we need to calculate the noise level in the total map. If we sum $N$ independent channels each with noise $\sigma_{\text {ch }}$, the final noise will be equal to $\sigma_{\mathrm{ch}} \sqrt{N}$. However, using a mask, the number of summed channels is different from pixel to pixel, moreover the data cubes in our sample have been Hanning-smoothed and adjacent channels are not independent (see Verheijen \& Sancisi 2001). To have a final estimate of the noise in the total map, we follow the approach of Verheijen \& Sancisi (2001) and Lelli, Verheijen \& Fraternali (2014a): we constructed a signal-to-noise map and we defined as the $3 \sigma$ pseudo-level $\left(\sigma_{3 \mathrm{~T}}\right)$ the mean value of the pixels with a $\mathrm{S} / \mathrm{N}$ between 2.75 and 3.25. We fit our kinematic model only to the portion of the data cube within the contour defined by $\sigma_{3 \mathrm{~T}}$ and avoiding to use rings that do not intercept, around the major axis, $\mathrm{H}_{\mathrm{I}}$ emission coming from the disc. This ensures a robust estimate of the galactic kinematics avoiding regions of the galaxy dominated by the noise and with poor information about the gas rotation. The $3 \sigma$ pseudo-noise levels $\sigma_{3 \mathrm{~T}}$ are listed in Table 1 (Column 9), while the maximum radii $R_{\max }$ used in the fit are reported in Table 2 (Columns 1 and 2).

\subsection{Data cube fit}

Given the assumptions we made (Section 3.2.1), we are left with two geometrical $(i, \mathrm{PA})$ and two kinematic $\left(V_{\text {rot }}\right.$ and $\left.\sigma_{v}\right)$ parameters.

Equation (1) shows that the rotational velocity and the geometrical parameters are coupled: in particular they become degenerate for galaxies with rising rotation curves (Kamphuis et al. 2015) as it is the case for most of the dIrrs. As a consequence, the fitting algorithm tends to be sensitive to the initial guesses, so it is important to initialize the fit with educated guess values. We estimated $i$ and PA using both the $\mathrm{H}_{\mathrm{I}}$ data cube and the optical data as explained in Appendix A. The initial values for $V_{\text {rot }}$ and $\sigma_{v}$ have been set respectively to 30 and $8 \mathrm{~km} \mathrm{~s}^{-1}$ for all the galaxies. 3DB allows to use an azimuthal weighting function $w(\theta)$ (see equation 1) to 'weigh' the residuals non-uniformly across the rings (see Di Teodoro \& Fraternali 2015). We decided to use $w(\theta)=\cos \theta$ to weigh most the regions around the major axis, where most of the information on the galactic rotation lies. If we fit at the same time the four parameters, we can obtain rotation curves and velocity-dispersion profiles that show unphysical discontinuities due to the scatter noise of the geometrical parameters. For this reason, we run $3 \mathrm{DB}$ with the option TWOSTAGE (see Appendix D) turned on: first a fit with four free parameters is obtained, then the geometrical parameters are regularized with a polynomial, and finally a new fit of only $V_{\text {rot }}$ and $\sigma_{v}$ is obtained. We chose to use the lowest polynomial order allowed by the data: in practice, we set the geometrical parameters to a constant value, unless there is a clear evidence of radial trends of $i$ and/or of PA as for example in DDO 133 and in DDO 154. We assessed the existence of a radial trend and set the degree of the polynomial through the visual inspection of the radial profile of $i$ and/or PA, and also of the H I map, of the velocity field and of the data cube channels.

The result of the fit is compared by eye with the real data cube both analysing the position-velocity diagrams (PV, hereafter) along the major axis and the minor axis, and channel by channel. Figs 3 and 4 show the comparison in the channel maps between the observations and the best-fitting model found with 3DB for DDO 154 and NGC 2366: the emission of DDO 154 is reproduced quite well; the bestfitting model found for NGC 2366 traces the global kinematics but it is not able to reproduce the extended feature in the north-west of the galaxy (see e.g. channel at $120 \mathrm{~km} \mathrm{~s}^{-1}$ ). The presence of such kind of peculiarities makes the study of the dIrrs H I disc kinematics challenging and requires careful visual inspection. Whenever we notice that the model was not a good representation of the data, we restart the fit changing the initial guesses and/or varying the order of the polynomial regularization. 

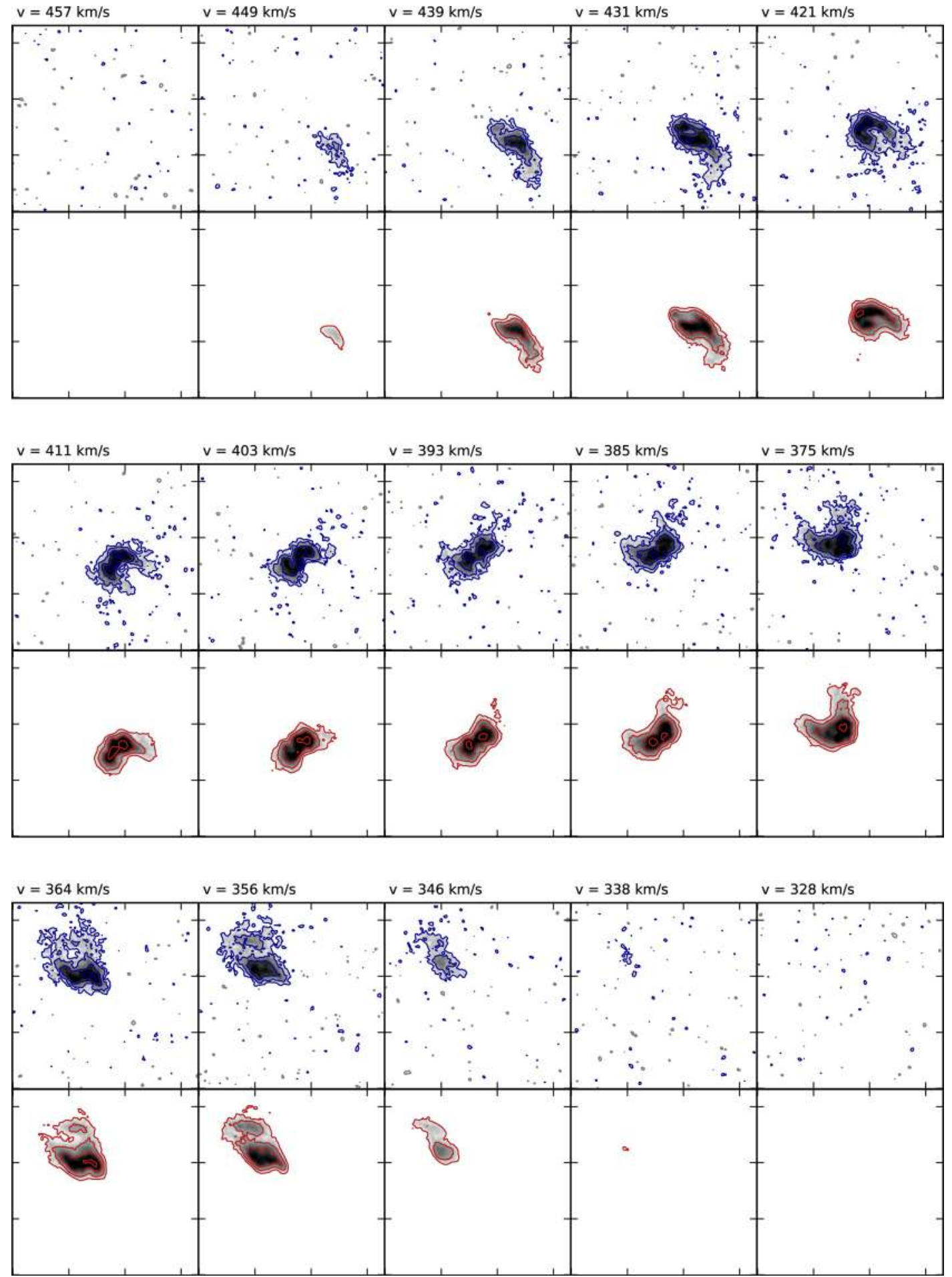

Figure 3. Channel maps showing the $\mathrm{H}$ I emission for DDO 154: data (top panels, blue contours) and the best-fitting model found with 3DB (bottom panels, red contours). The velocity of each channel is reported above the panels. The minimum contour is $3 \sigma_{\mathrm{ch}}$ (Column 8 in Table 1 ) and adjacent contours differ by a factor of 2 in emissivity; the grey contours in the data channels indicate the emission at $-3 \sigma_{\text {ch. }}$. The size of the field is about 14 arcmin $\times 14$ arcmin.

Concerning the errors on the estimated parameters, it is worth spending a few words about the differences between the standard $2 \mathrm{D}$ approach and $3 \mathrm{DB}$. In the first case, the errors are a combination of (i) nominal errors coming from the tilted-ring fit (typically using a Levenberg-Marquardt algorithm) and (ii) the differences between the fit of the approaching and receding sides. It is well known that the nominal errors are very small. The uncertainties are dominated by the difference between the approaching and receding sides, an 'asymmetric-error' that is not straightforward to quantify. 3DB uses a Monte Carlo method to estimate the errors further exploring the parameter space around the best-fitting solutions (see Di Teodoro \& Fraternali 2015 for further details); therefore, our quoted errors should be a robust and statistically significant measure of the uncertainties of the kinematic parameters. 

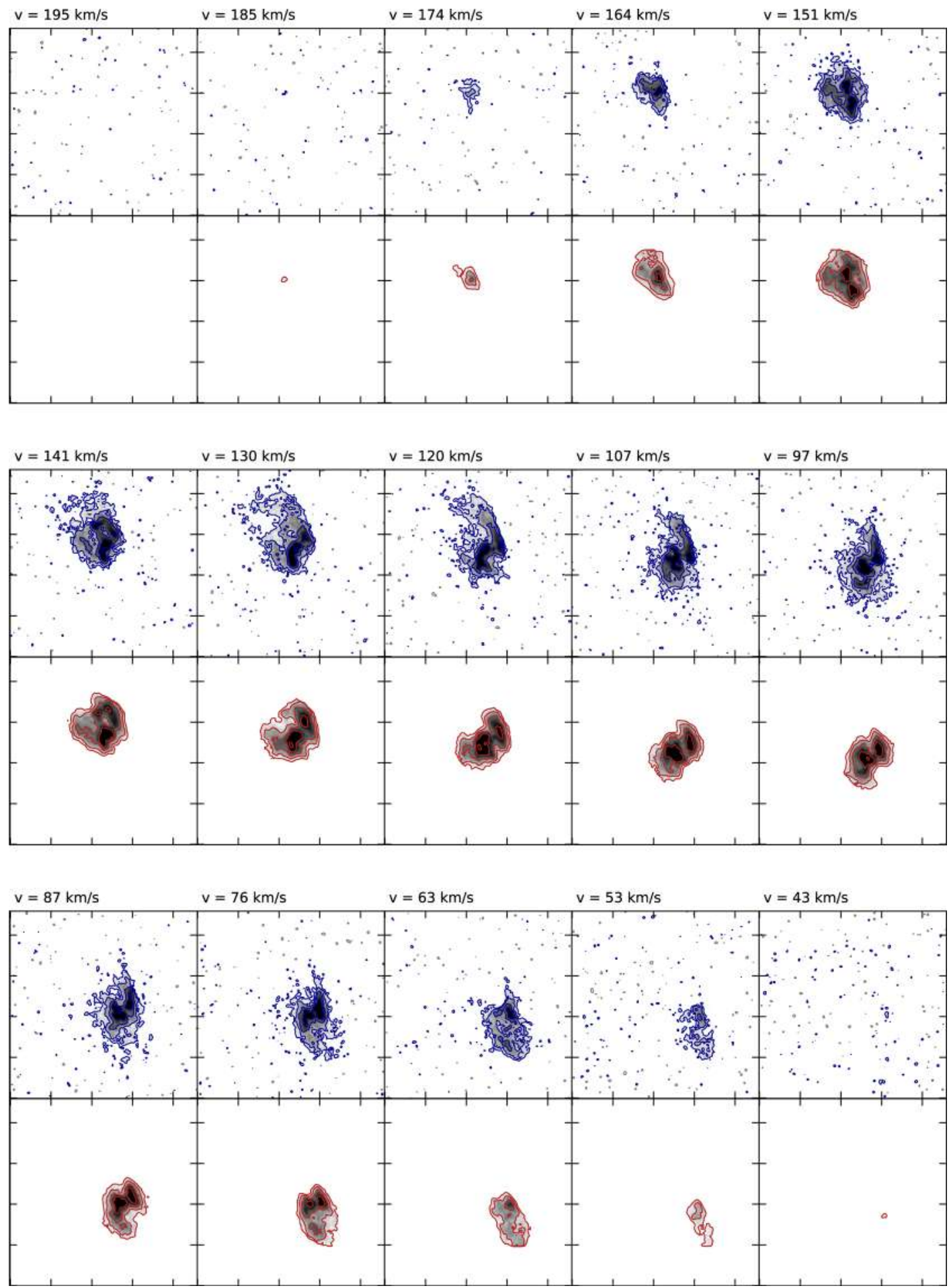

Figure 4. Same as Fig. 3 but for NGC 2366. The size of the field is about $20 \operatorname{arcmin} \times 20 \operatorname{arcmin}$.

Given that we use the errors estimated with $3 \mathrm{DB}$, we did not fit separately the approaching and receding halves of the galaxy as often done in the classical 2D methods. The only exception is DDO 126: in this case the best-fitting model found with $3 \mathrm{DB}$ using the whole galaxy does not give a good representation of the data cube because of the strong kinematic asymmetries (see Section 5).

\subsection{The final circular velocity}

Assuming that the gas is in equilibrium, the rotation velocity of the gas $\left(V_{\text {rot }}\right)$ can be related to the galactic gravitational potential $(\Phi)$ through the radial component of the momentum equation

$\frac{1}{\rho} \frac{\partial \rho \sigma_{\mathrm{v}}^{2}}{\partial R}=-\frac{\partial \Phi}{\partial R}+\frac{V_{\mathrm{rot}}^{2}}{R}$ 
where $\rho$ is the volumetric density of the gas and $\sigma_{v}$ is the velocity dispersion. Therefore, the observed $V_{\text {rot }}$ is not a direct tracer of the galactic potential when the pressure support term $\left(\rho \sigma_{\mathrm{v}}^{2}\right)$ due to the random gas motions is non-negligible.

\subsubsection{Asymmetric-drift correction}

Equation (6) can be re-written as

$V_{\mathrm{c}}^{2}-V_{\mathrm{rot}}^{2}=-\frac{R}{\rho} \frac{\partial \rho \sigma_{\mathrm{v}}^{2}}{\partial R}=-R \sigma_{\mathrm{v}}^{2} \frac{\partial \ln \left(\rho \sigma_{\mathrm{v}}^{2}\right)}{\partial R}=V_{\mathrm{A}}^{2}$,

where $V_{\mathrm{c}}=\sqrt{R \frac{\partial \Phi}{\partial R}}$ is the circular velocity and $V_{\mathrm{A}}$ is the asymmetric drift, which becomes increasingly important at large radii and is heavily dependent on the value of the velocity dispersion. When the rotation velocity is much higher than the velocity dispersion the asymmetric-drift correction is negligible. This is the case in spiral galaxies where usually $V_{\text {rot }} / \sigma_{v}>10$ (e.g. de Blok et al. 2008). Instead, for several dIrrs the rotation velocity is comparable to the velocity dispersion, making the asymmetric-drift correction indispensable for an unbiased estimate of the galactic potential.

The volumetric density in the equatorial plane $(z=0)$ is proportional to the ratio of the intrinsic surface density and the vertical scaleheight $\left(z_{\mathrm{d}}\right)$; therefore, the asymmetric drift $V_{\mathrm{A}}^{2}$ is given by

$V_{\mathrm{A}}^{2}=-R \sigma_{\mathrm{v}}^{2} \frac{\partial \ln \left(\sigma_{\mathrm{v}}^{2} \Sigma_{\text {int }} z_{\mathrm{d}}^{-1}\right)}{\partial R}$.

We can ignore the term with the radial derivative of $z_{\mathrm{d}}$ assuming that the thickness of the gaseous layer is independent of radius (but see Section 7.1). Furthermore, assuming that the $\mathrm{H}_{\mathrm{I}}$ disc is thin, the ratio between the intrinsic and the observed surface density is just the cosine of $i$ (equation 4). Under these assumptions, from equation (8) we derive the classical formulation of the asymmetricdrift correction (see e.g. O15):

$V_{\mathrm{A}}^{2}=-R \sigma_{\mathrm{v}}^{2} \frac{\partial \ln \left(\sigma_{\mathrm{v}}^{2} \Sigma_{\mathrm{obs}} \cos i\right)}{\partial R}$.

Except for DDO 168, NGC 1569 and UGC 8508, all the analysed galaxies have a constant $i$ and the cosine in equation (9) can also be ignored.

\subsubsection{Application to real data}

Fluctuations of the observed surface density and of the measured velocity dispersion at similar radii can have dramatic effects on the numerical calculation of the radial derivative in equation (9). As a consequence, the final asymmetric-drift correction can be very scattered causing abrupt variations in the final estimate of the circular velocity. For this reason, we decided to use functional forms to describe both the velocity dispersion and the argument of the logarithm in equation (9). The velocity dispersion is regularized with a polynomial $\sigma_{\mathrm{p}}\left(R, n_{\mathrm{p}}\right)$ with degree $n_{\mathrm{p}}$ lower than 3 . If there is not a clear radial trend, we consider a fixed velocity dispersion taking the median of $\sigma_{v}(R)$. The radial variation of the velocity dispersion is usually small; therefore, the radial trend of $\Sigma_{\text {int }} \sigma_{\mathrm{v}}^{2}$ is dominated by the behaviour of the surface density: this falls off exponentially at large radii, while in the centre it is almost constant or it shows an inner depression. In analogy with Bureau \& Carignan (2002), we chose to fit $\Sigma_{\text {int }} \sigma_{\mathrm{v}}^{2}$ with the function

$f(R)=f_{0}\left(\frac{R_{\mathrm{c}}}{\operatorname{arcsec}}+1\right)\left(\frac{\mathrm{R}_{\mathrm{c}}}{\operatorname{arcsec}}+\mathrm{e}^{\frac{R}{R_{\mathrm{d}}}}\right)^{-1}$, where $f_{0}$ is a normalization coefficient, and $R_{\mathrm{c}}$ and $R_{\mathrm{d}}$ are characteristic radii. The function $f$ is characterized by an exponential decline at large radii and by an inner core almost equal to $f_{0}$. Read et al. (2016c) showed that equation (10) is a good compromise between a pure exponential that overestimates the asymmetric-drift correction in the inner radii, and a functional form with an inner depression that can produce unphysical negative values of $V_{\text {rot }}^{2}$. Combining equations (9) and (10), we can write the asymmetric-drift correction as

$V_{\mathrm{A}}^{2}=R \frac{\sigma_{\mathrm{p}}^{2}\left(R, n_{\mathrm{p}}\right) \mathrm{e}^{\frac{R}{R_{\mathrm{d}}}}}{R_{\mathrm{d}}}\left(\frac{\mathrm{R}_{\mathrm{c}}}{\operatorname{arcsec}}+\mathrm{e}^{\frac{R}{R_{\mathrm{d}}}}\right)^{-1}$.

\subsubsection{Error estimates}

We can calculate the final errors on $V_{\mathrm{c}}$ by applying the propagation of errors to equation (7) to obtain

$\delta_{\mathrm{c}}=\frac{\sqrt{V_{\mathrm{rot}}^{2} \delta_{\mathrm{rot}}^{2}+V_{\mathrm{A}}^{2} \delta_{\mathrm{A}}^{2}}}{V_{\mathrm{c}}}$,

where $\delta_{\text {rot }}$ is the error found with $3 \mathrm{DB}$ and $\delta_{\mathrm{A}}$ is the uncertainty associated with the estimated values of the asymmetric-drift term. Usually $\delta_{\mathrm{A}}$ is simply ignored (e.g. Bureau \& Carignan 2002; O15), thus the uncertainties on the final corrected rotation curve are equal to $\delta_{\text {rot }}$. This is a reasonable assumption if the rotational terms in equation (12) are dominant, but for some galaxies in our sample (e.g. DDO 210) the final circular rotation curve is heavily dependent on the asymmetric-drift correction. In these cases, it is important that $\delta_{\mathrm{c}}$ includes the uncertainties introduced by the operations described in Section 4.3.2. We decided to estimate $\delta_{\mathrm{c}}$ with a Monte Carlo approach:

(i) First we make $N$ realizations of the radial profile of both the velocity dispersion and of the surface density. For each sampling radius $R$, the values of a single realization $\sigma_{\mathrm{v}}^{i}(R)$ or $\Sigma_{\text {int }}^{i}(R)$ are extracted randomly from a normal distribution with the centre and the dispersion taken respectively from the values and errors of the parent populations.

(ii) For each of the $N$ realizations, we apply the method described in Section 4.3.2 to obtain the asymmetric-drift correction at each sampling radius $V_{\mathrm{A}}^{i}(R)$.

(iii) The final asymmetric-drift correction is calculated as $V_{\mathrm{A}}(R)=\operatorname{median}_{i}\left(V_{\mathrm{A}}^{i}(R)\right)$, where each $V_{\mathrm{A}}^{i}$ is obtained with equation (11). The associated errors are $\delta_{\mathrm{A}}(R)=K \times \operatorname{MAD}\left(V_{\mathrm{A}}^{i}(R)\right)$, where the MAD is the median absolute deviation around the median. The factor $K$ links the MAD with the standard deviation of the sample ( $K \approx 1.48$ for a normal distribution). We chose to use the median and the MAD because they are less biased by the presence of outliers with respect to the mean and the standard deviation.

We found that $N=1000$ is enough to obtain a good description of the error introduced by the asymmetric-drift correction.

\subsubsection{Final notes}

The method described in the above sections has been built to make the asymmetric-drift correction terms as smooth as possible. This ensures that our final rotation curves are not affected by nonphysical noise related to the derivative term in equation (9). The drawback of this approach is that intrinsic scatter on $V_{\mathrm{A}}$ is hidden and the final errors $\delta_{\mathrm{A}}$ could be several times larger than the point-to-point scatter. However, we are confident that the quoted 
values of $\delta_{\mathrm{A}}$ truly trace the degree of uncertainties introduced by the asymmetric-drift correction at different radii. In galaxies where the final circular velocity is totally dependent on the asymmetricdrift correction (e.g. DDO 210, see Section 5 and Fig. 16) the scatter in $V_{\text {rot }}$ could be smoothed out, but in these cases also the final errors are dominated by the errors on the asymmetric drift and $\delta_{\mathrm{c}}$ is still a good representation of the global uncertainties.

In some cases the velocity dispersion found with $3 \mathrm{DB}$ is not well constrained, so there are galaxies where at some radii $\sigma_{v}$ is discrepant or peculiar (e.g. very small error) with respect to the global trend. In general, the presence of a single 'rogue' $\sigma_{v}$ (e.g. DDO 87, Fig. 10 or DDO 133, Fig. 13) does not have a significant influence on the estimate of the $V_{\mathrm{A}}$ and on the median of $\sigma_{v}$ (see Table 2). However, if the discrepant $\sigma_{v}$ is in a peculiar position (e.g. the last radius in DDO 210, Fig. 16) or the region with 'rogues' is extended by more than one ring (e.g. DDO 216, empty circles in Fig. 17), the correction for the asymmetric drift and the estimate of the circular velocities are biased. In these cases, we exclude the radii with discrepant $\sigma_{v}$ both from the calculation of the asymmetric-drift correction and from the calculation of the median.

\section{RESULTS}

For each analysed galaxy, we produced a summary plot of its properties (Figs 5-21). Each plot is divided in four boxes: Box A shows the rotation curve, the circular-velocity profile and the correction for the asymmetric drift; Box B shows the velocity dispersion, the intrinsic surface density, $i$ and PA obtained with 3DB; Box C shows the total H I map and the velocity field; Box D shows the PV diagrams along the major and the minor axis for both model and data. The physical radii have been calculated assuming the distance in Table 1 (Column 1). A detailed description of the plot layout can be found in Appendix B. For reference, Table 2 lists the assumptions and the initial guesses used in 3DB (Columns 1-7) and a summary of the results of the data cube fit (Columns 8-11). Here, below we report notes on individual galaxies. In the following notes, $i_{\text {ini }}$ and $\mathrm{PA}_{\text {ini }}$ indicate the initial guesses for $i$ and PA, respectively (see Columns 6 and 7 in Table 2).

(i) CVnIdwA. The H I morphology of CVnIdwA (Fig. 5) looks regular, but the orientation of the $\mathrm{HI}$ iso-density contours is quite different with respect to the direction of the velocity gradient. The emission of the stellar disc is very patchy, so we estimated the geometrical parameters of the $\mathrm{H}$ I disc using 3DB (see Appendix A).

(ii) DDO 47. The HI disc of DDO 47 (Fig. 6) is nearly faceon, while the stellar disc looks highly inclined at $64^{\circ}$ (Hunter \& Elmegreen 2006). An inclination of $64^{\circ}$ is not compatible with the H I contours of the total map and the stellar disc probably hosts a bar-like structure (Georgiev, Karachentsev \& Tikhonov 1997). Therefore, we decided to estimate $i_{\text {ini }}$ and $\mathrm{PA}_{\text {ini }}$ by fitting ellipses on the contours of the total H I map (see Appendix A). The discrepancy between the galactic centre estimated with the $\mathrm{H}$ I contours and the optical centre is very small ( 0.7 arcsec in RA and 2 arcsec in Dec.), so we decided to use the $\mathrm{H}$ I centre. The best-fitting $i$ (see Table 2) is consistent with previous works $\left(35^{\circ}\right.$ in Gentile et al. $2005,30^{\circ}$ in de Blok \& Bosma 2002 and Stil \& Israel 2002b) and seems a reasonable upper limit for this galaxy (see ellipses in Box $\mathrm{C}$ in Fig. 6). The final rotation curve shows a flattening at $R<120^{\prime \prime}$ arcsec that could be the sign of the presence of strong non-circular motion. Moreover, in the approaching half of the galaxy there is a large hole that can be clearly seen both on the total map and on the PV along the major axis: the presence of such hole could further bias the estimate of the gas rotation. For these reasons, we retain the velocities for $R<120$ arcsec not completely reliable (empty circles in Box A in Fig. 6).

(iii) DDO 50. The gaseous disc of DDO 50 (Fig. 7) is quite peculiar: it is 'drilled' by medium-large $\mathrm{H}$ I holes ranging from $100 \mathrm{pc}$ to $1.7 \mathrm{kpc}$ (Puche et al. 1992) and it also shows clumpy regions with high density. Therefore, the $\mathrm{H}_{\mathrm{I}}$ surface density evaluated across the rings could be very discontinuous with very high peaks and/or regions without emission. As a consequence, the quoted errors on the intrinsic surface density profile (see Section 4.1.1) are large and they are probably overestimating the real statistical uncertainties, especially in the inner disc $(R<3 \mathrm{kpc})$. Despite this peculiarity, the large-scale kinematics is quite regular and typical of a flat rotation curve. The estimates of the $i$ and PA are difficult given that this galaxy is nearly face-on. The value of $i$ estimated with the isophotal fitting of the optical disc $\left(47^{\circ}\right)$ is too high to be compatible with the flattening of the $\mathrm{H}$ I contours that favour an $i<40^{\circ}$. The best agreement between the data cube and the model has been found using initial values of $30^{\circ}$ for $i_{\text {ini }}$ and $180^{\circ}$ for $\mathrm{PA}_{\text {ini }}$. The centre was set using the coordinates of the centre of the stellar disc (Hunter \& Elmegreen 2006).

(iv) DDO 52. The best-fitting $i$ of DDO 52 (Fig. 8) tends to be more edge-on at the end of the disc. However, the analysis of the channels and of the PVs indicates that the model with a constant $i$ gives a better representation of the data.

(v) DDO 53. The stellar disc and the H I disc of DDO 53 (Fig. 9) are misaligned and the galaxy is nearly face-on. As a consequence, $i$ and PA are very difficult to constrain: we tried different values in the range $30^{\circ}-50^{\circ}$ for $i_{\text {ini }}$ and $100^{\circ}-140^{\circ}$ for $\mathrm{PA}_{\text {ini }}$. The best match with the data has been found using $i_{\text {ini }}=35^{\circ}$ and $\mathrm{PA}=120^{\circ}$, but the rotational and the circular velocities should be taken with caution. In the northern part of the galaxy, there is some extra emission possibly connected with an inflow/outflow: this region is clearly visible in the PV along the minor axis around -50 arcsec at a velocity of about $5 \mathrm{~km} \mathrm{~s}^{-1}$.

(vi) DDO 87. The morphology of DDO 87 (Fig. 10) is clearly irregular in the inner part, but the outer disc looks more regular. We decided to set the initial guesses for the PA using 3DB (see Appendix A). We tried different initial guesses for $i$ : the best representation of the data has been obtained with $i_{\text {ini }}=40^{\circ}$ and $\mathrm{PA}_{\text {ini }}=240^{\circ}$, approximately $15^{\circ}$ lower than the orientation of the optical disc (Hunter \& Elmegreen 2006).

(vii) DDO 101. The HI disc of DDO 101 (Fig. 11) is extended only slightly beyond the optical disc and the $\mathrm{H}_{\mathrm{I}}$ emission is almost constant with some high density structures around $1.0 \mathrm{kpc}$. Notice that the estimates of the distance for this galaxy are very uncertain (ranging from 5 to $16 \mathrm{Mpc}$ ) since they all rely on a poor distance estimator (Tully-Fisher relation, e.g. Karachentsev, Makarov \& Kaisina 2013).

(viii) DDO 126. DDO 126 (Fig. 12) shows kinematic asymmetries, so we separately run $3 \mathrm{DB}$ also on the approaching and the receding halves of the galaxy. Beyond $1.5 \mathrm{kpc}$ the fit on the whole galaxy gives a good representation of the data cube and the errors found with $3 \mathrm{DB}$ are larger than (or comparable to) the differences due to the kinematic asymmetries (black circles in Box A in Fig. 12). The inner regions are less regular and the best model has been found taking the mean between the approaching and receding rotation curves (empty circles in Box A in Fig. 12), while the errors have been calculated as half the difference between the two values following the recipe of Swaters (1999). 
(A)

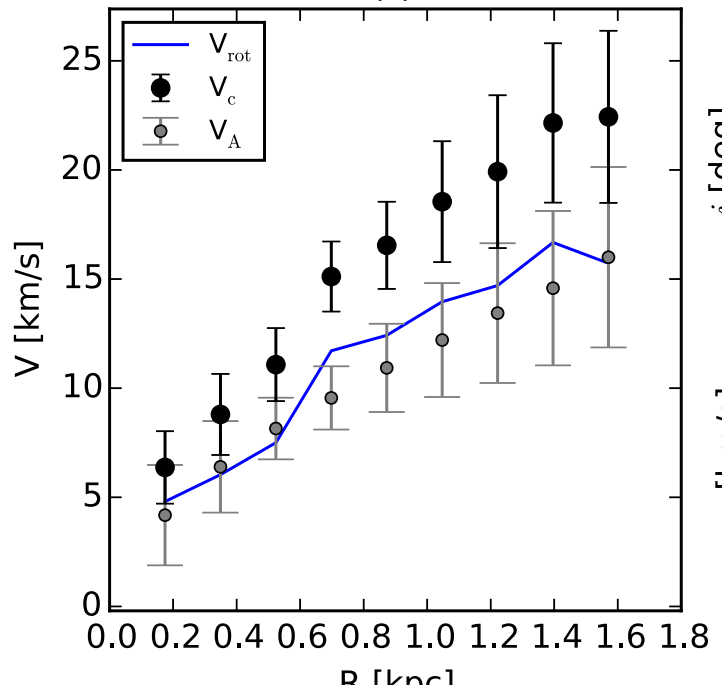

(C)
CVn I dwA

(B)

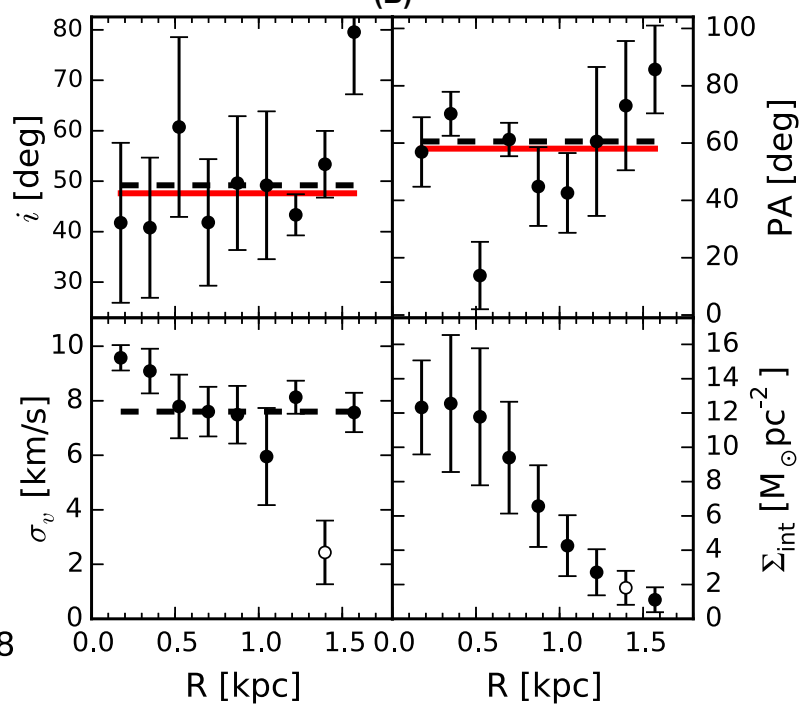

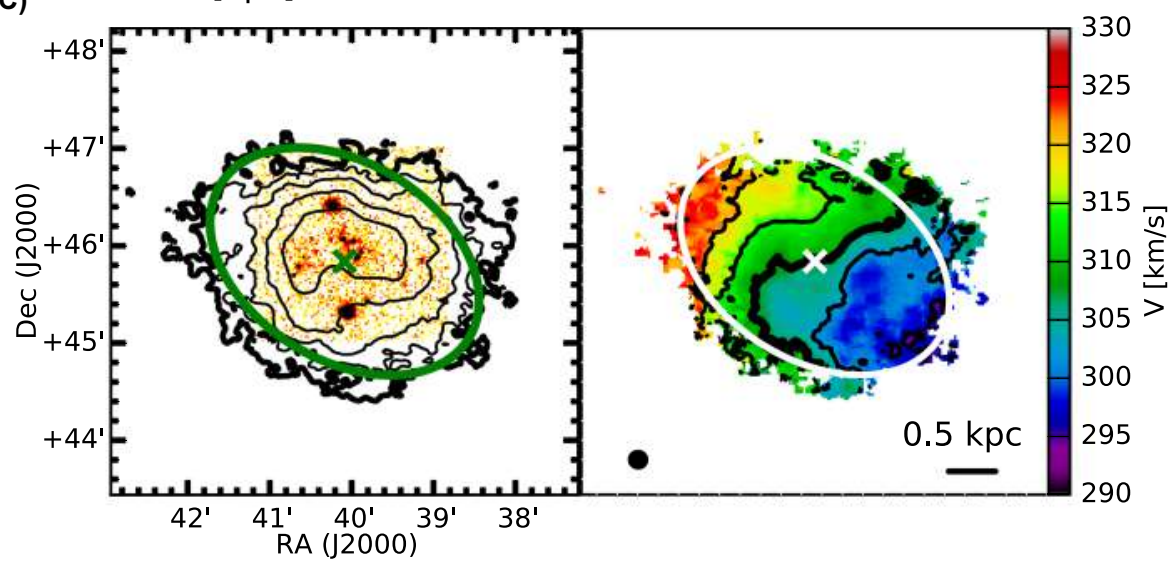

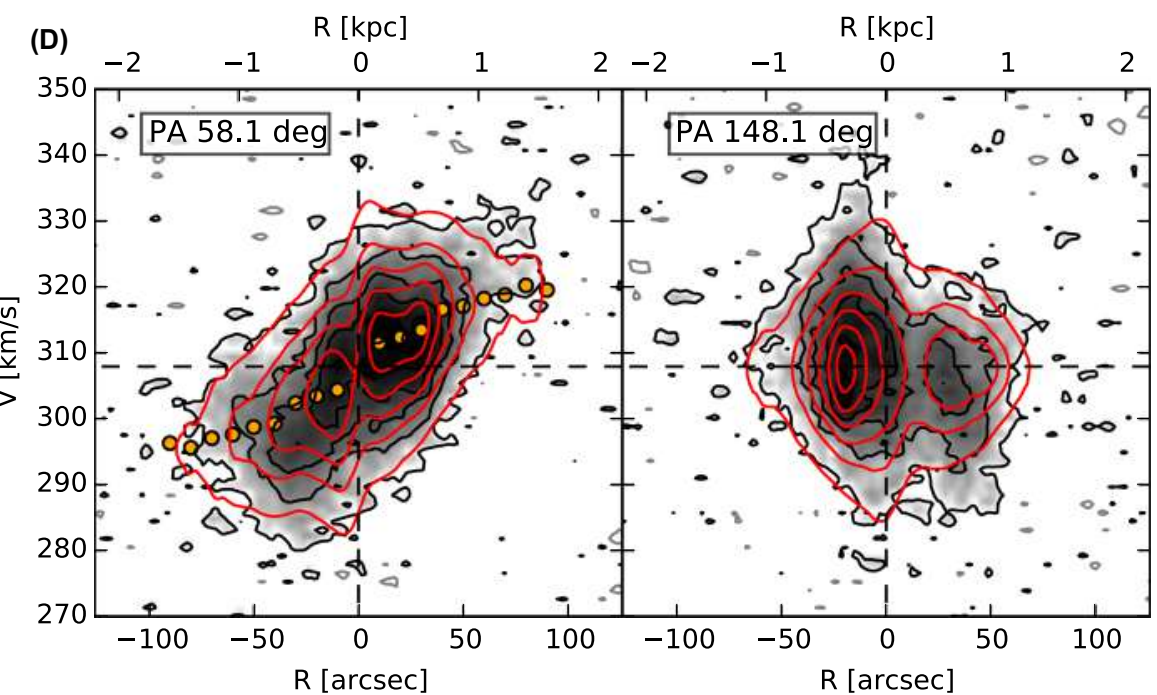

Figure 5. See caption in Appendix B. Notes - C (left-hand panel): contours at $2^{n} \sigma_{3 \mathrm{~T}}$ and $\sigma_{3 \mathrm{~T}}=0.96 \mathrm{M}_{\odot} \mathrm{pc}^{-2}$ (thick contour), stellar map in SDSS $r$ band (Baillard et al. 2011); C (right-hand panel): contours at $V_{\text {sys }} \pm \Delta V$, where $\Delta V=6.0 \mathrm{~km} \mathrm{~s}^{-1}$ and $V_{\text {sys }}=307.9 \mathrm{~km} \mathrm{~s}^{-1}$ (thick contour); D: contours at $(2+4 n) \sigma_{\mathrm{ch}}$, where $\sigma_{\mathrm{ch}}=0.63 \mathrm{mJy} \mathrm{bm}^{-1}$, the grey contours are at $-2 \sigma_{\mathrm{ch}}$. 


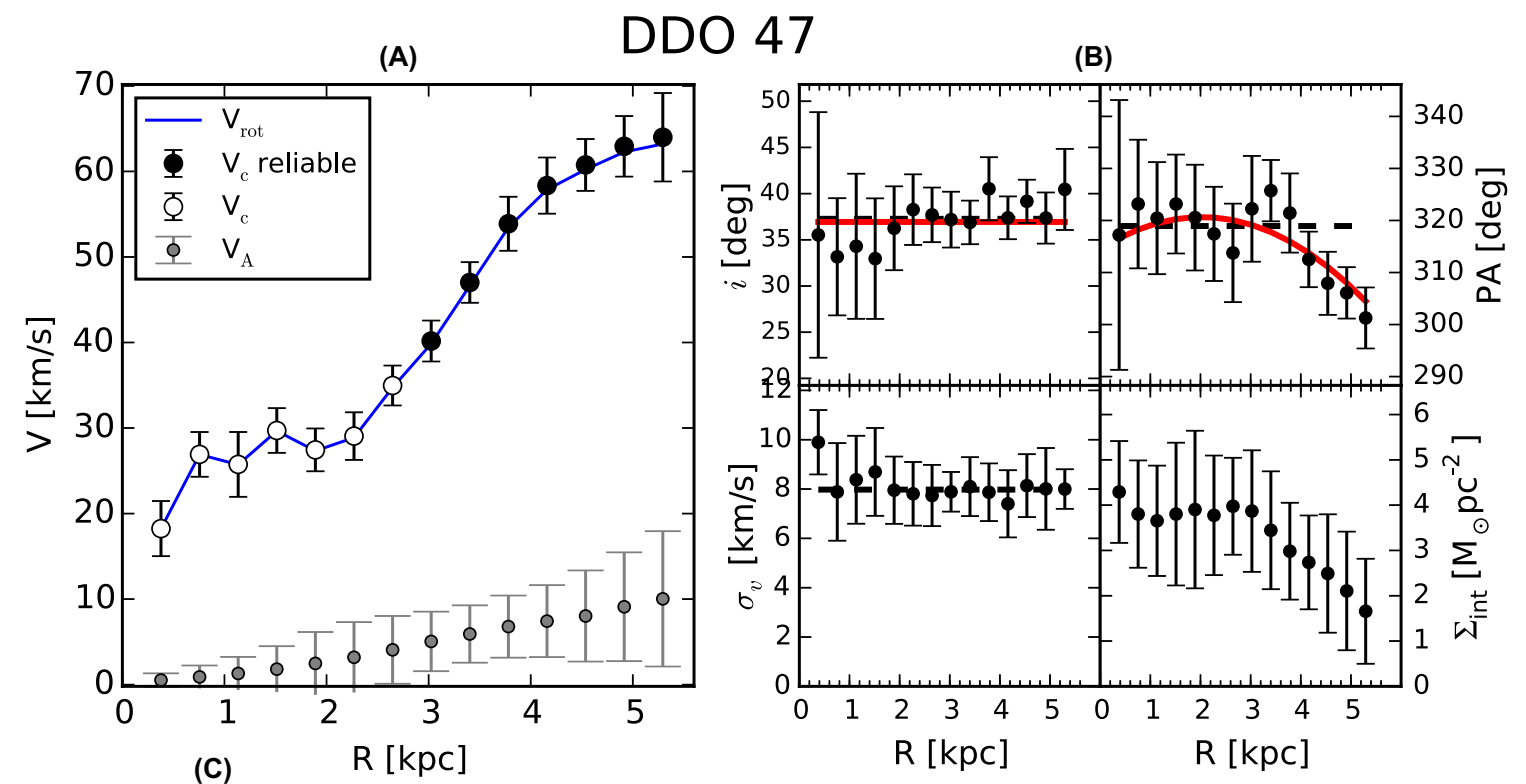

(C) $\quad \mathrm{R}[\mathrm{kpc}]$
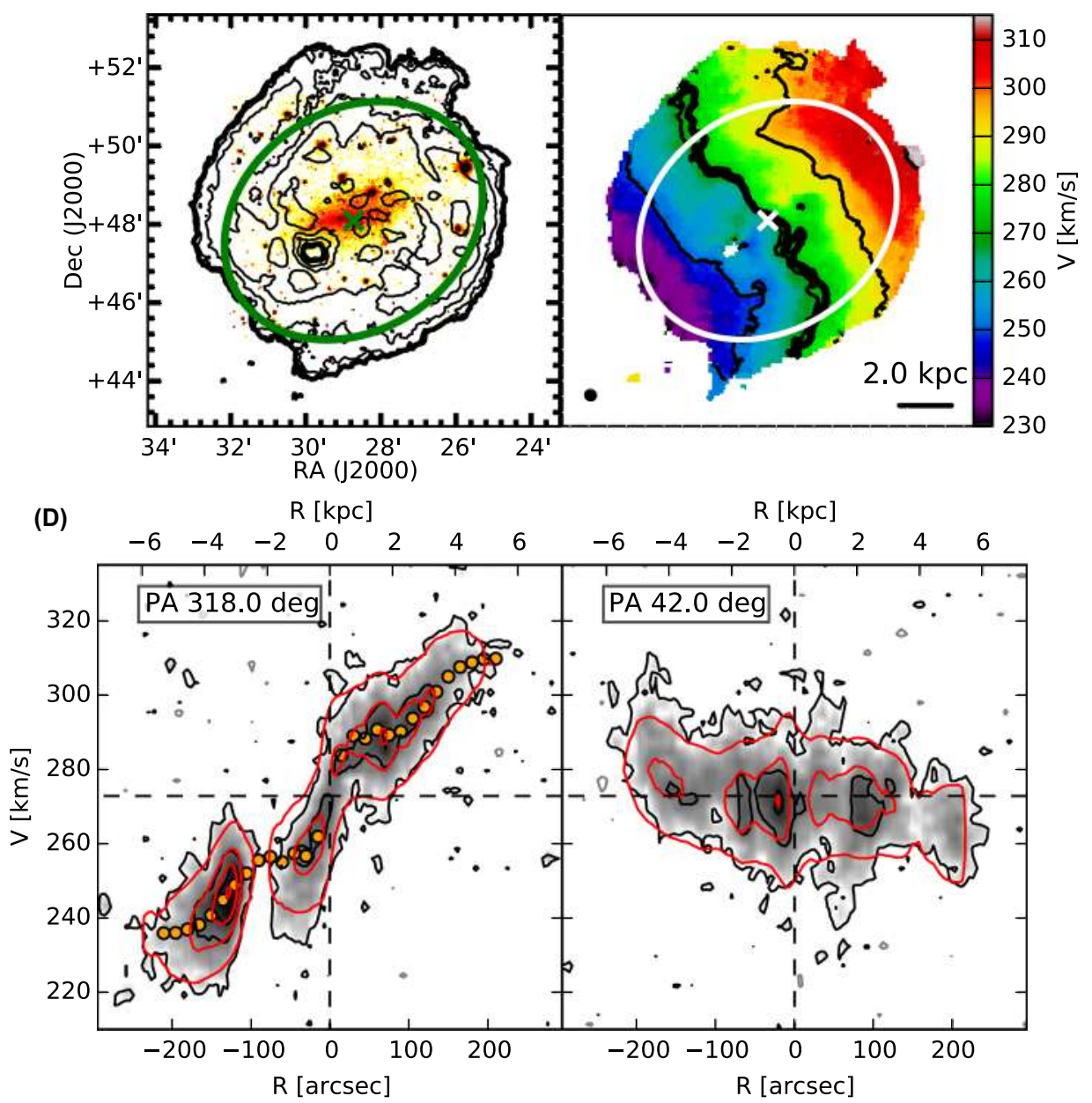

Figure 6. See caption in Appendix B. Notes - A: the empty circles indicate the region in which the estimate of the rotation curve can be biased by small H $\mathrm{I}$ regions at anomalous velocity along the major axis (see the text for further details); $\mathrm{C}$ (left-hand panel): contours at $2^{n} \sigma_{3 \mathrm{~T}}$ and $\sigma_{3 \mathrm{~T}}=0.41 \mathrm{M}_{\odot} \mathrm{pc}^{-2}$ (thick contour), stellar map in SDSS $r$ band (Baillard et al. 2011); C (right-hand panel): contours at $V_{\text {sys }} \pm \Delta V$, where $\Delta V=20.0 \mathrm{~km} \mathrm{~s}{ }^{-1}$ and $V_{\text {sys }}=272.8 \mathrm{~km} \mathrm{~s}{ }^{-1}$ (thick contour); D: contours at $(2+6 n) \sigma_{\mathrm{ch}}$, where $\sigma_{\mathrm{ch}}=0.61 \mathrm{mJy} \mathrm{bm}^{-1}$, the grey contours are at $-2 \sigma_{\mathrm{ch}}$. 


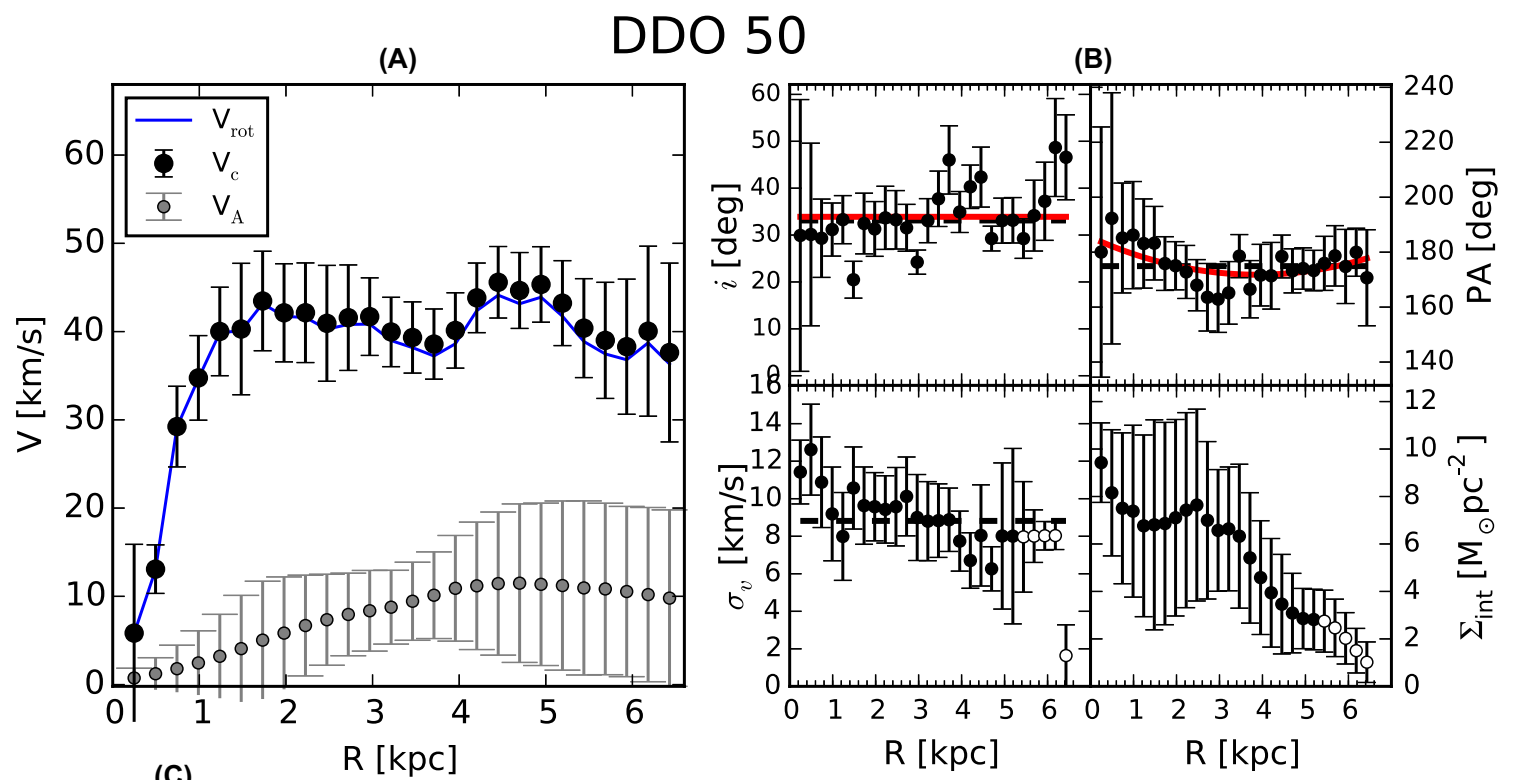

(C)
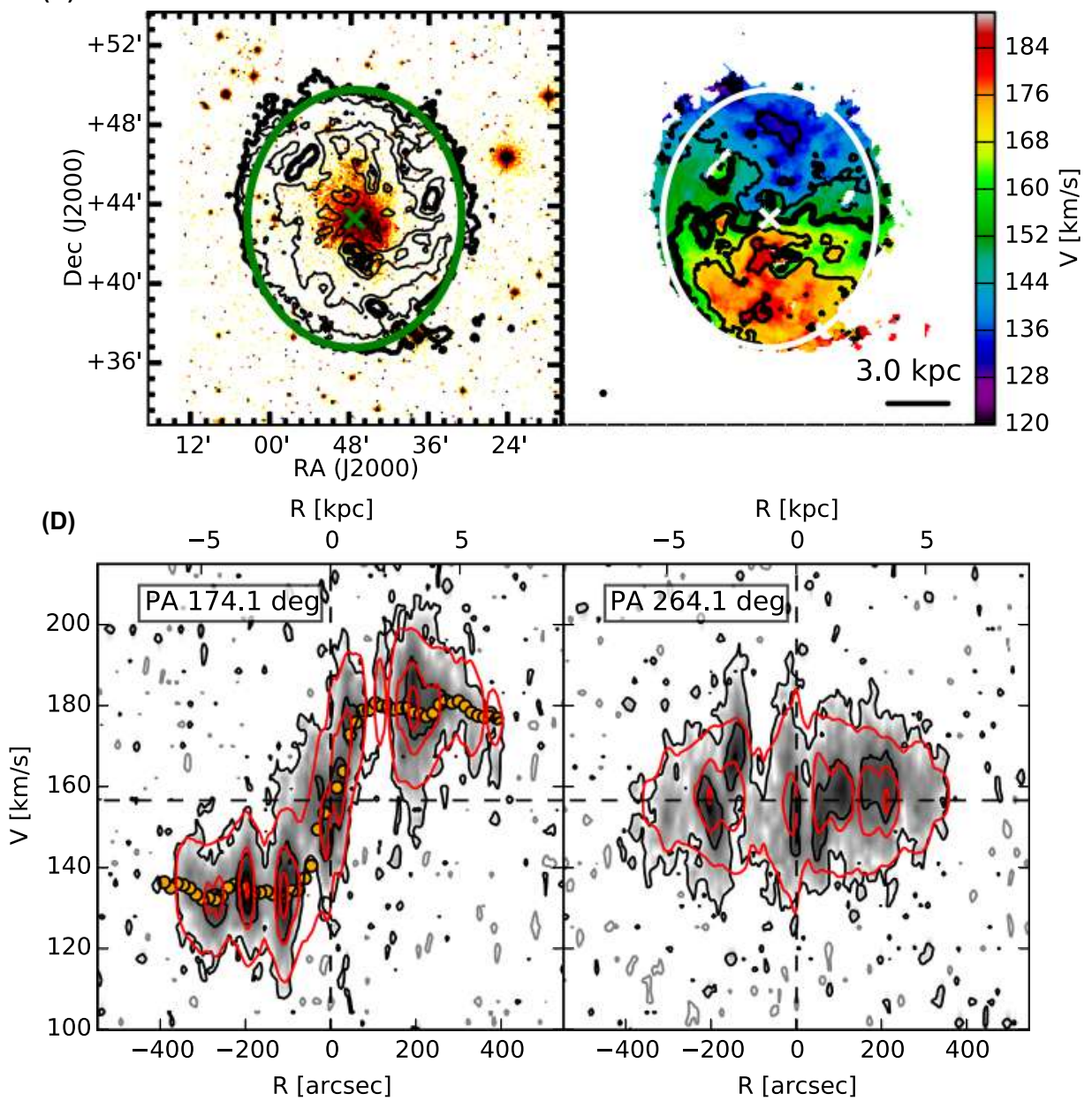

Figure 7. See caption in Appendix B. Notes - C (left-hand panel): contours at $3^{n} \sigma_{3 \mathrm{~T}}$ and $\sigma_{3 \mathrm{~T}}=0.65 \mathrm{M}_{\odot} \mathrm{pc}^{-2}$ (thick contour), stellar map in $R$ band from Cook et al. (2014); $\mathrm{C}$ (right-hand panel): contours at $V_{\text {sys }} \pm \Delta V$, where $\Delta V=12.0 \mathrm{~km} \mathrm{~s}^{-1}$ and $V_{\text {sys }}=156.7 \mathrm{~km} \mathrm{~s}^{-1}$ (thick contour); D: contours at (2+7n) $\sigma_{\text {ch }}$, where $\sigma_{\mathrm{ch}}=0.82 \mathrm{mJy} \mathrm{bm}^{-1}$, the grey contours are at $-2 \sigma_{\mathrm{ch}}$.

(ix) DDO 133. The HI disc of DDO 133 (Fig. 13) has a regular kinematics, although there is evidence of non-circular motions, especially in the region of the stellar bar (Hunter et al. 2011). The final PA found with $3 \mathrm{DB}$ is about $20^{\circ}$ in the inner part of the disc $(R<0.5 \mathrm{kpc})$ and it becomes almost zero in the outer disc. We decided to take the radial trend of the PA into account with a fourth order polynomial. 
(A)

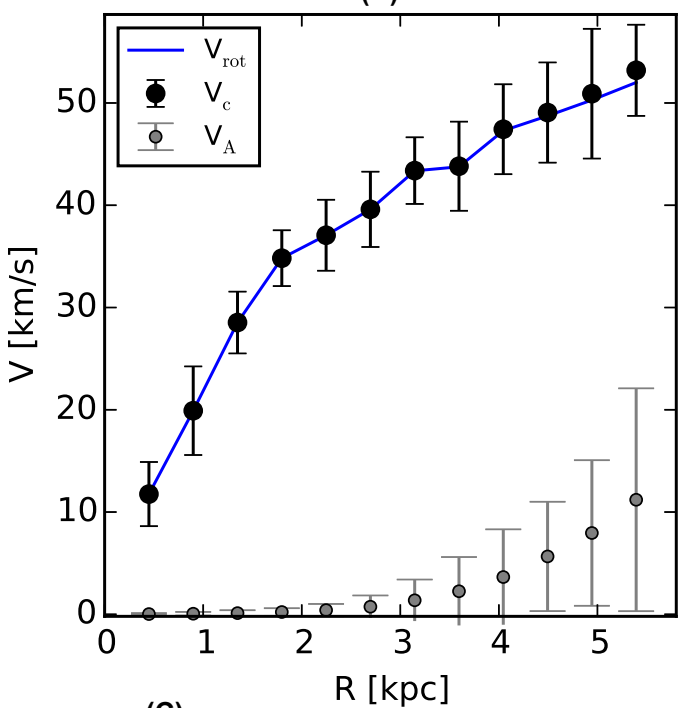

(C)
DDO 52

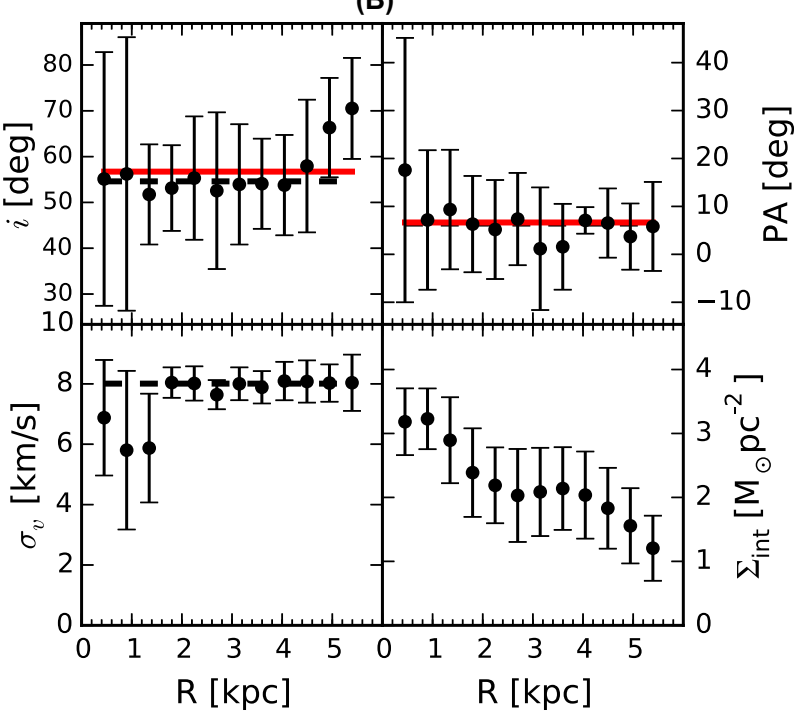

(B)

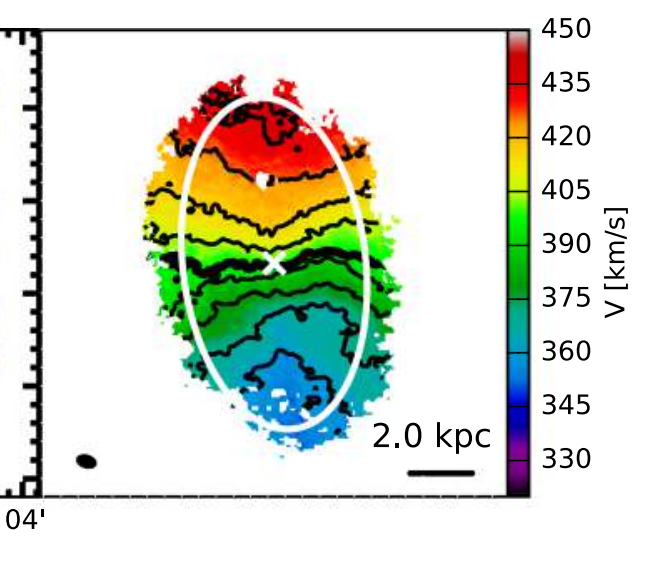

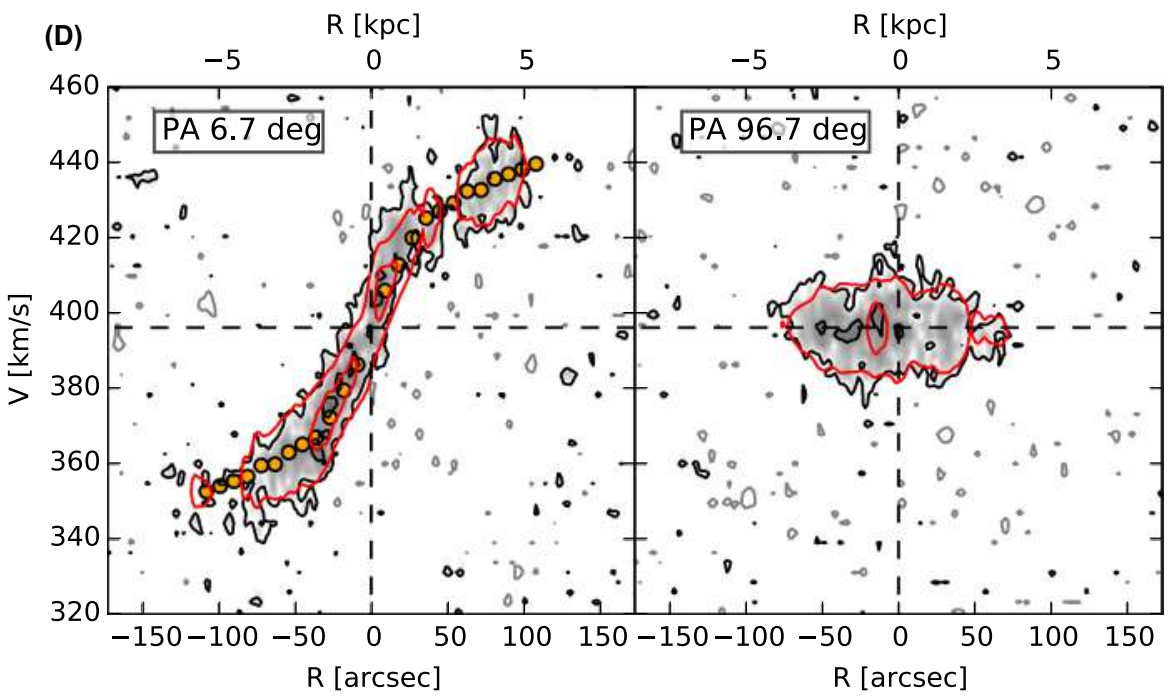

Figure 8. See caption in Appendix B. Notes - C (left-hand panel): contours at $2^{n} \sigma_{3 \mathrm{~T}}$ and $\sigma_{3 \mathrm{~T}}=1.20 \mathrm{M}_{\odot}$ pc ${ }^{-2}$ (thick contour), stellar map in SDSS $r$ band (Baillard et al. 2011); C (right-hand panel): contours at $V_{\text {sys }} \pm \Delta V$, where $\Delta V=10.0 \mathrm{~km} \mathrm{~s}^{-1}$ and $V_{\text {sys }}=396.2 \mathrm{~km} \mathrm{~s}{ }^{-1}$ (thick contour); D: the contours start from $(2+4 n) \sigma_{\mathrm{ch}}$, where $\sigma_{\mathrm{ch}}=0.46 \mathrm{mJy} \mathrm{bm}^{-1}$, the grey contours are at $-2 \sigma_{\mathrm{ch}}$.

(x) DDO 154. The Hi morphology and the kinematics of DDO 154 (Fig. 14) is quite regular. However, the contours of the H I map clearly show a radial trend of the PA, as confirmed by the values found with $3 \mathrm{DB}$. In order to obtain a good description of the radial trend of the PA, we used a fourth order polynomial. However, the best-fitting polynomial shows a very steep gradient (about $25^{\circ}$ ) in a very small region (less than $1 \mathrm{kpc}$ ) that is not justified by the data (red dashed line in Box B in Fig. 14). The points located in this 
(A) $\quad$ DDO 53

(B)
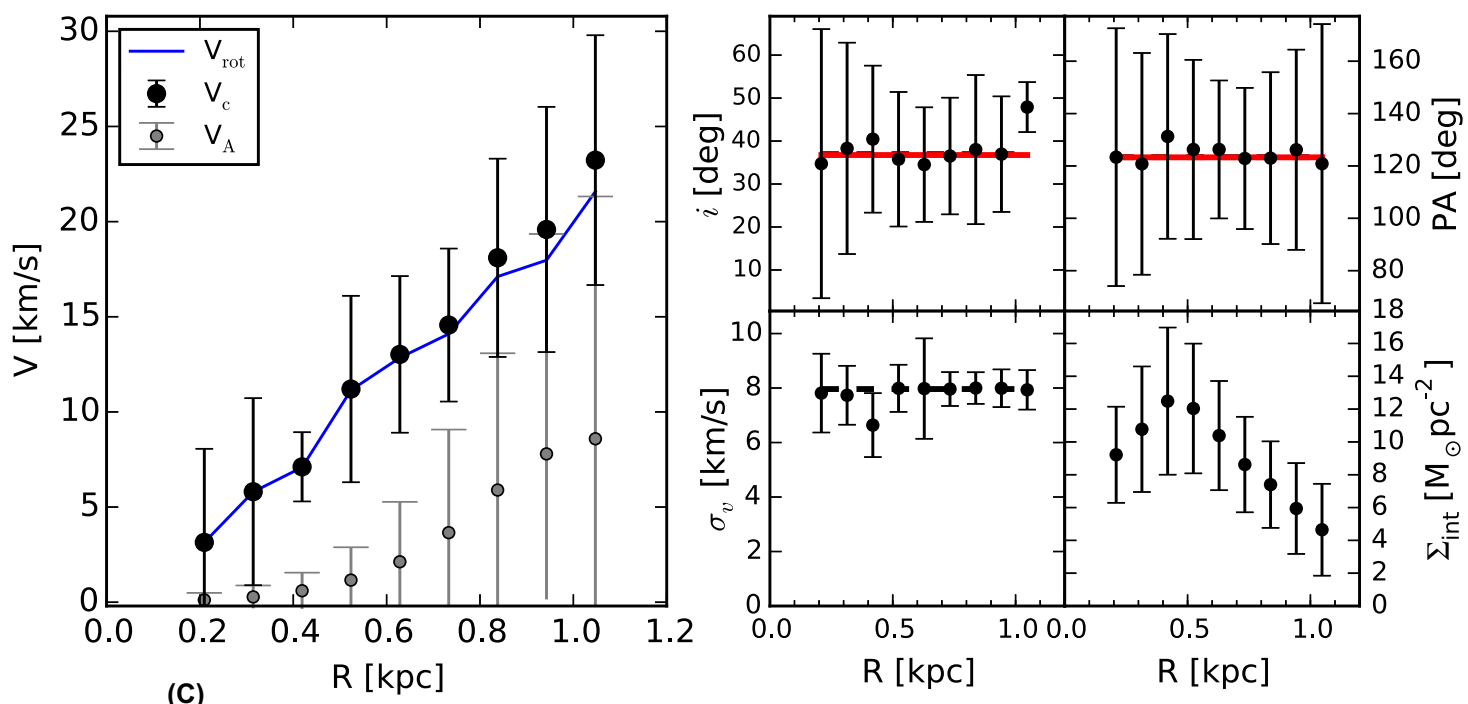

(C)

$\mathrm{R}[\mathrm{kpc}]$

$\mathrm{R}[\mathrm{kpc}]$

$\mathrm{R}[\mathrm{kpc}]$

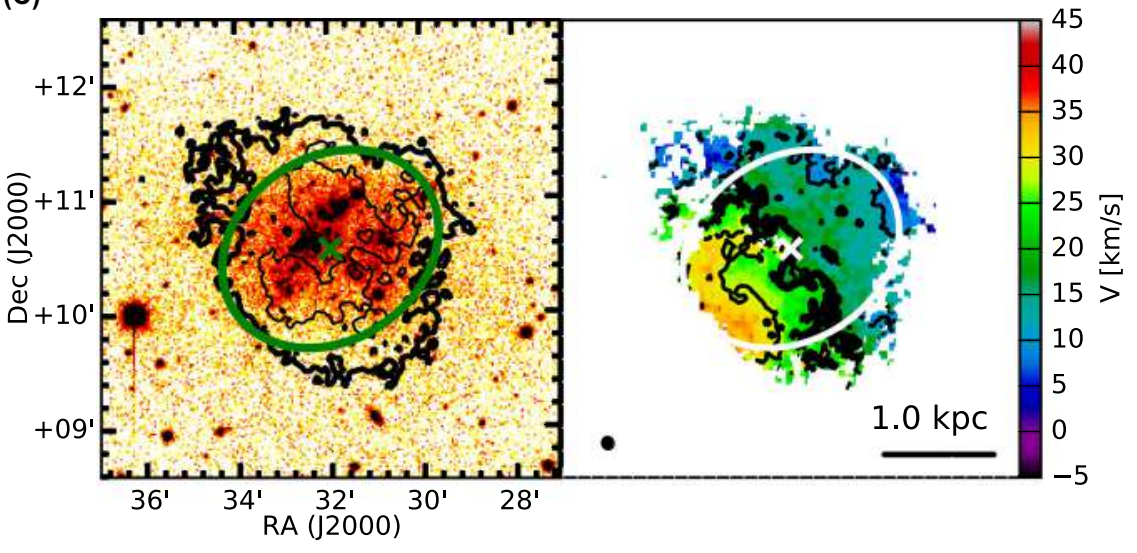

$\mathrm{R}[\mathrm{kpc}]$

$\mathrm{R}[\mathrm{kpc}]$

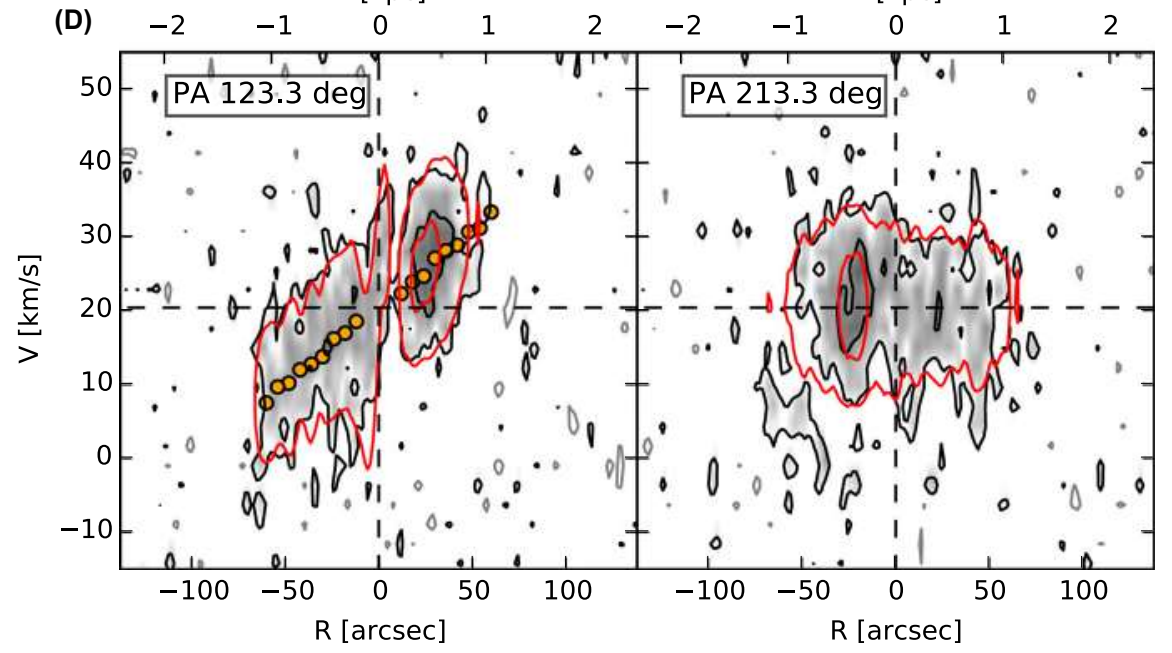

Figure 9. See caption in Appendix B. Notes $-\mathrm{C}$ (left-hand panel): contours at $2^{n} \sigma_{3 \mathrm{~T}}$ and $\sigma_{3 \mathrm{~T}}=3.80 \mathrm{M}_{\odot} \mathrm{pc}^{-2}$ (thick contour), stellar map in $R$ band from Cook et al. (2014); C (right-hand panel): contours at $V_{\text {sys }} \pm \Delta V$, where $\Delta V=8.0 \mathrm{~km} \mathrm{~s}^{-1}$ and $V_{\text {sys }}=20.4 \mathrm{~km} \mathrm{~s}{ }^{-1}$ (thick contour); D: contours at $(2+4 n) \sigma_{\text {ch }}$, where $\sigma_{\mathrm{ch}}=0.57 \mathrm{mJy} \mathrm{bm}^{-1}$, the grey contours are at $-2 \sigma_{\mathrm{ch}}$.

region are compatible with a constant $\mathrm{PA}$, so we decided to fix it to the mean values of the first three points (see Box B in Fig. 14). As in $\mathrm{O} 15$, we do not confirm the almost Keplerian fall-off of the rotation curve claimed by Carignan \& Purton (1998) beyond $5 \mathrm{kpc}$. The channel maps are shown in Fig. 3. (xi) DDO 168. The velocity gradient of DDO 168 (Fig. 15) is misaligned with respect to both the $\mathrm{H}_{\mathrm{I}}$ and the stellar disc. Moreover, the presence of a prominent bar visible both in the stellar disc (Hunter \& Elmegreen 2006) and in the inner part of the $\mathrm{H}_{\mathrm{I}}$ disc makes the initial estimate of the geometrical parameters very 
(A) $\quad$ DDO 87

(B)
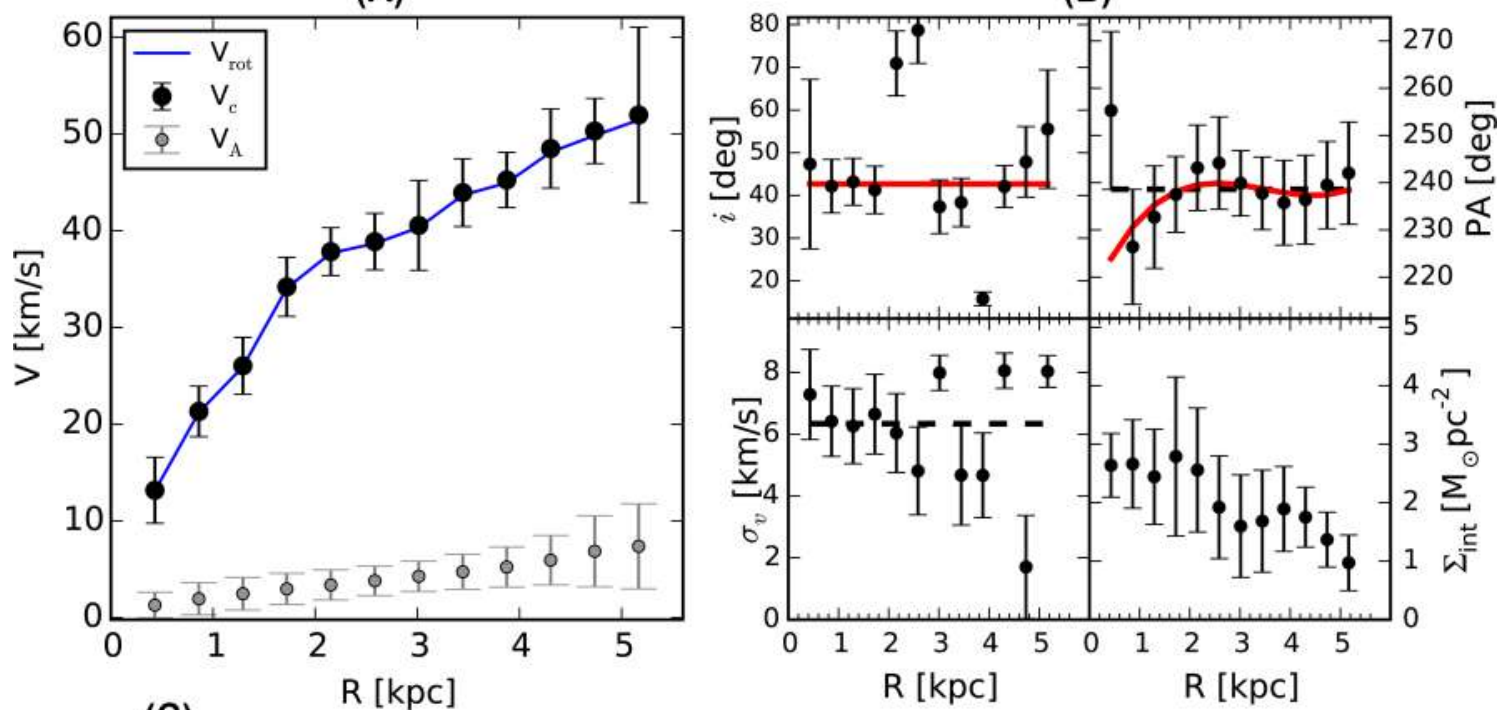

(C)
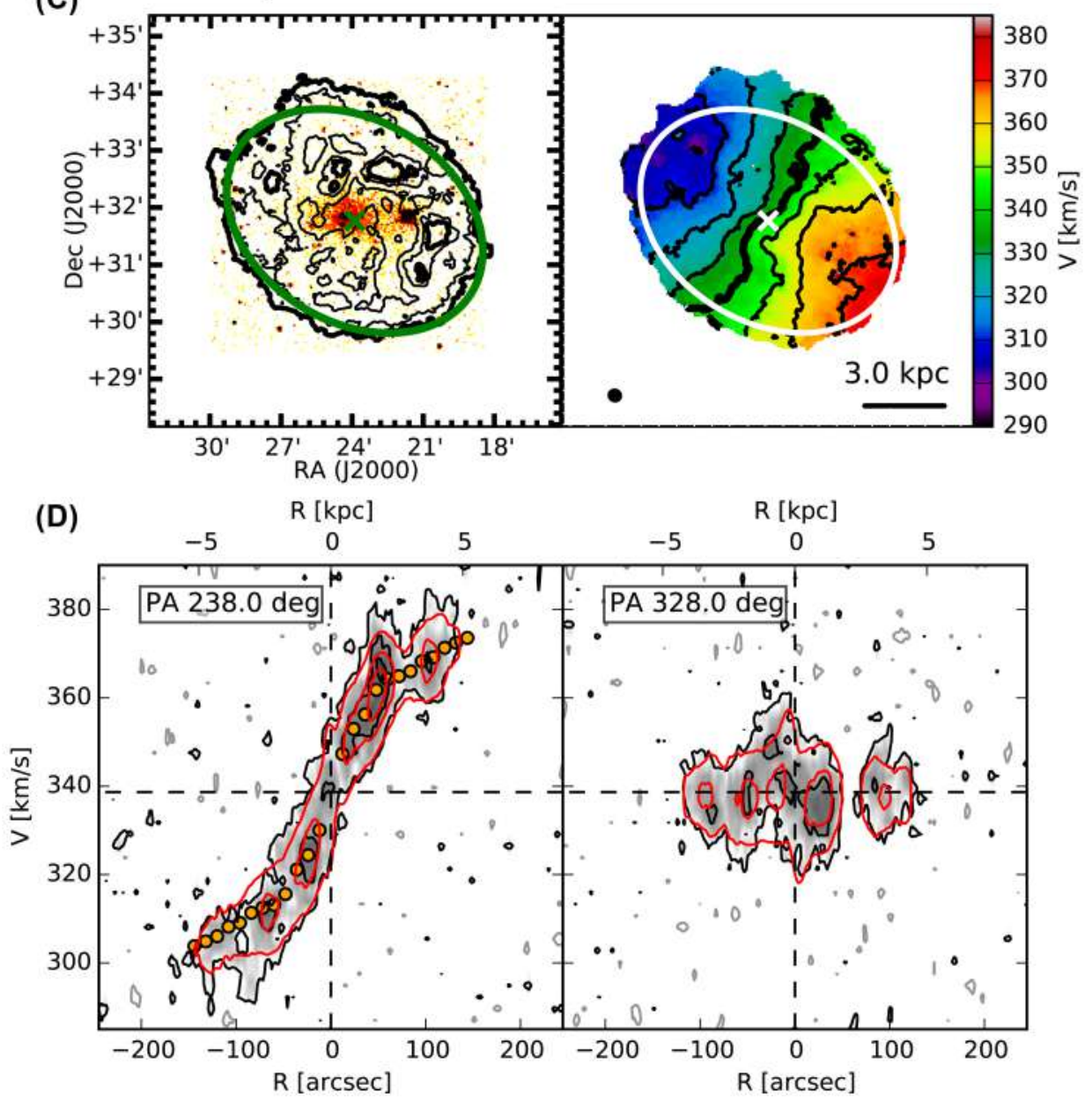

Figure 10. See caption in Appendix B. Notes - C (left-hand panel): contours at $2^{\mathrm{n}} \sigma_{3 \mathrm{~T}}$ and $\sigma_{3 \mathrm{~T}}=0.90 \mathrm{M}_{\odot} \mathrm{pc}^{-2}$ (thick contour), stellar map in SDSS $r$ band (Baillard et al. 2011); $\mathrm{C}$ (right-hand panel): Contours at $V_{\text {sys }} \pm \Delta V$, where $\Delta V=10.0 \mathrm{~km} \mathrm{~s}^{-1}$ and $V_{\text {sys }}=338.7 \mathrm{~km} \mathrm{~s}^{-1}$ (thick contour); D: contours at $(2+4 n) \sigma_{\mathrm{ch}}$, where $\sigma_{\mathrm{ch}}=0.51 \mathrm{mJy} \mathrm{bm}^{-1}$, the grey contours are at $-2 \sigma_{\mathrm{ch}}$.

uncertain. We obtained a first estimate of the PA using the 2D tiltedring fitting of the velocity field shown in Fig. 15: the resultant PA decreases from $300^{\circ}$ to about $270^{\circ}$. We used these values as initial guesses for all sampling radii in $3 \mathrm{DB}$ (see Appendix A). The centre was set to the value found with $3 \mathrm{DB}$ that roughly corresponds to the optical centre. We tried different values for $i_{\text {ini }}$ between $40^{\circ}$ and $70^{\circ}$ : the best reproduction of the data cube has been obtained with $i_{\text {ini }}=60^{\circ}$. The final results still show a variation of PA that we 
(A) DDO 101

(B)
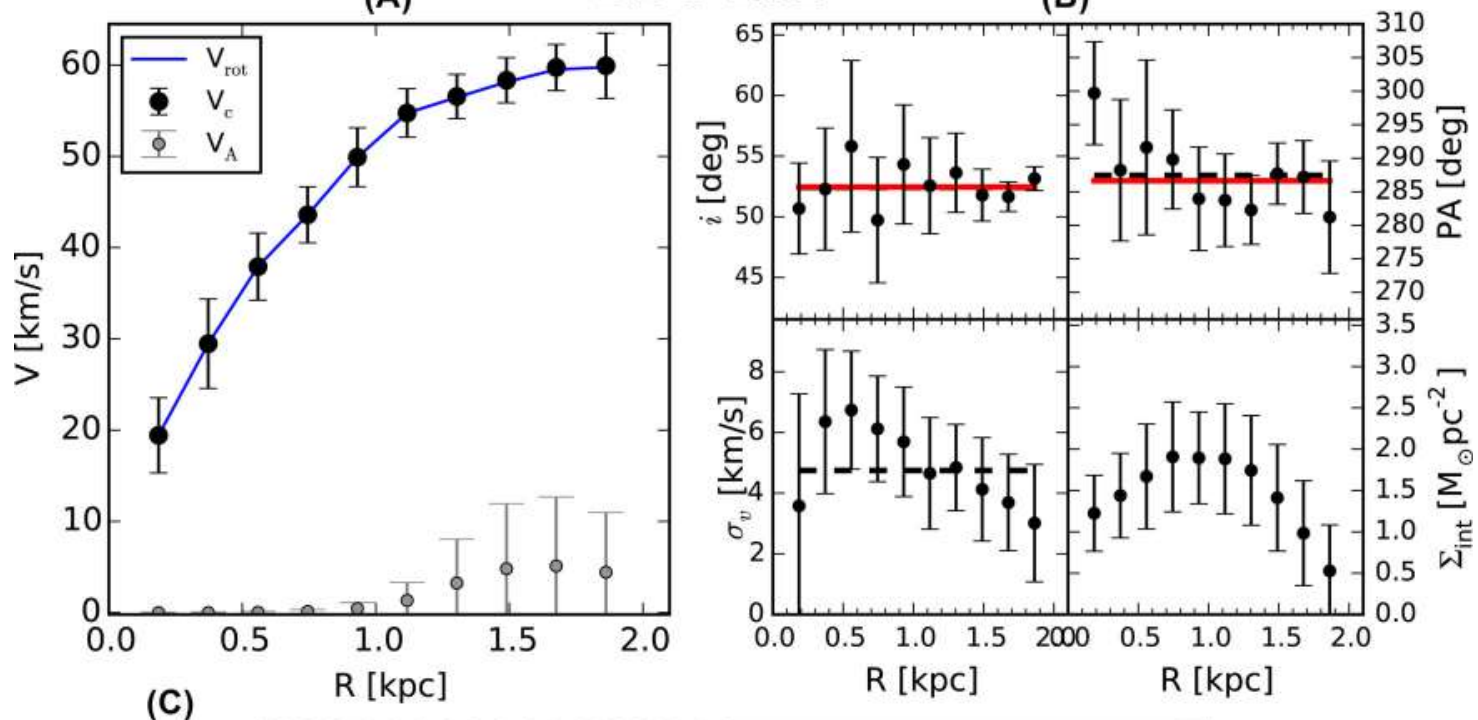

(C)
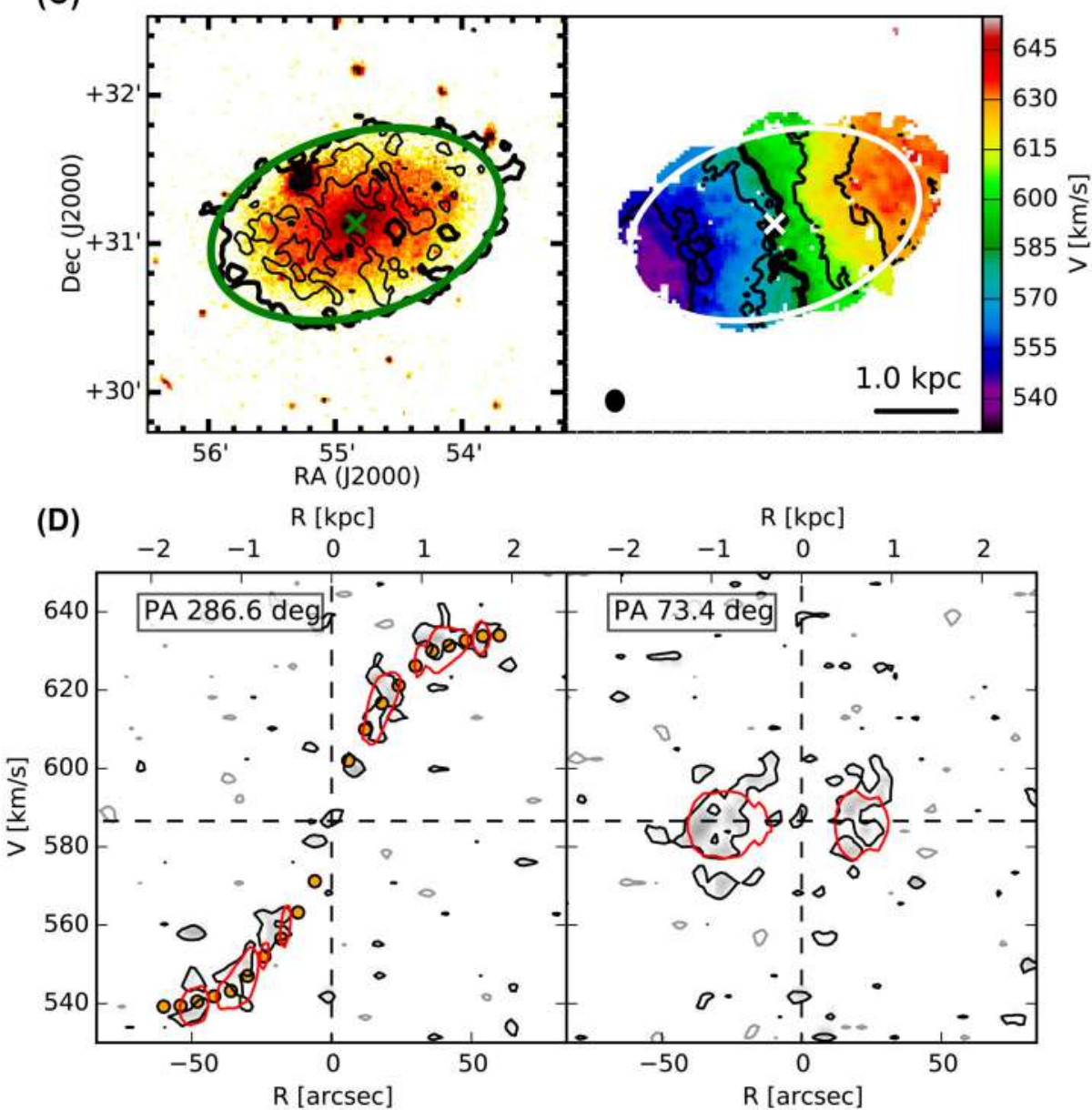

Figure 11. See caption in Appendix B. Notes - C (left-hand panel): contours at $4^{n} \sigma_{3 \mathrm{~T}}$ and $\sigma_{3 \mathrm{~T}}=0.90 \mathrm{M}_{\odot} \mathrm{pc}^{-2}$ (thick contour), stellar map in SDSS $r$ band (Baillard et al. 2011); $\mathrm{C}$ (right-hand panel): contours at $V_{\mathrm{sys}} \pm \Delta V$, where $\Delta V=20.0 \mathrm{~km} \mathrm{~s}^{-1}$ and $V_{\mathrm{sys}}=586.6 \mathrm{~km} \mathrm{~s}{ }^{-1}$ (thick contour); D: contours at $(2+6 n) \sigma_{\mathrm{ch}}$, where $\sigma_{\mathrm{ch}}=0.50 \mathrm{mJy} \mathrm{bm^{-1 }}$, the grey contours are at $-2 \sigma_{\mathrm{ch}}$.

regularized with a second order polynomial. The outer disc of DDO 168 is quite irregular. There is extra emission at velocities close to $V_{\text {sys }}$ possibly related to inflow/outflow, while the distortions of the iso-velocity contours (Box C in Fig. 15) could be due to the presence of an outer warp. (xii) DDO 210. DDO 210 (also known as Aquarius dIrr, Fig. 16) is the least massive galaxy in our sample and it is classified as a transitional dwarf galaxy (McConnachie 2012 and reference therein). The $\mathrm{H}_{\mathrm{I}}$ map is quite peculiar with iso-density contours that are not elliptical. As a consequence, the estimate of the galactic centre using 
(A)

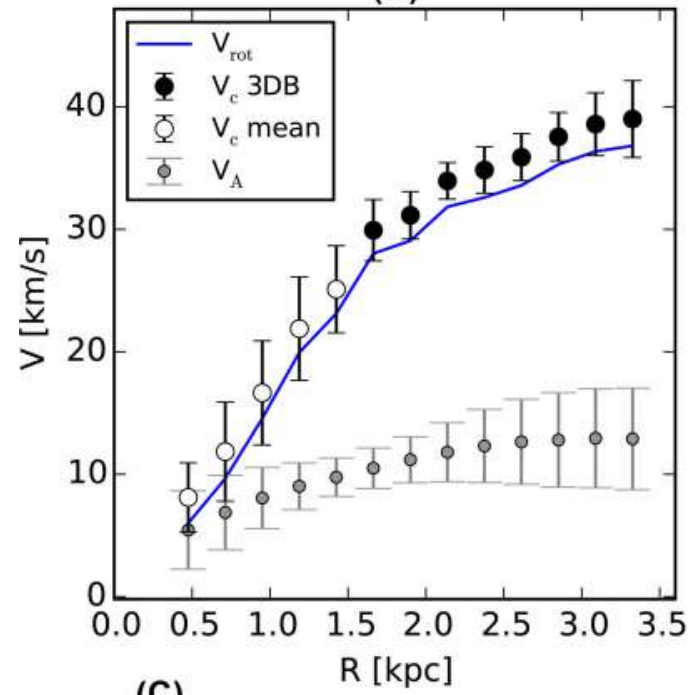

(B)

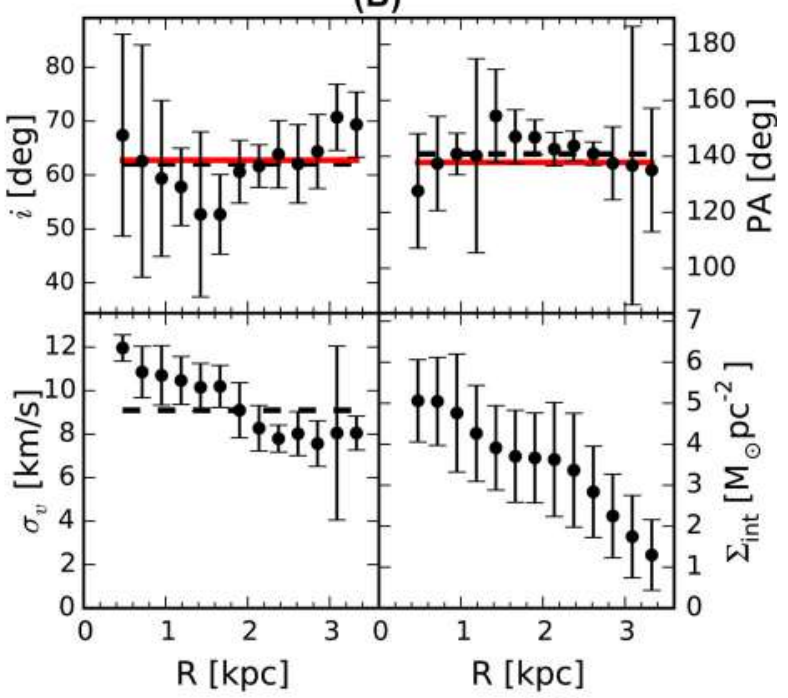

(C)
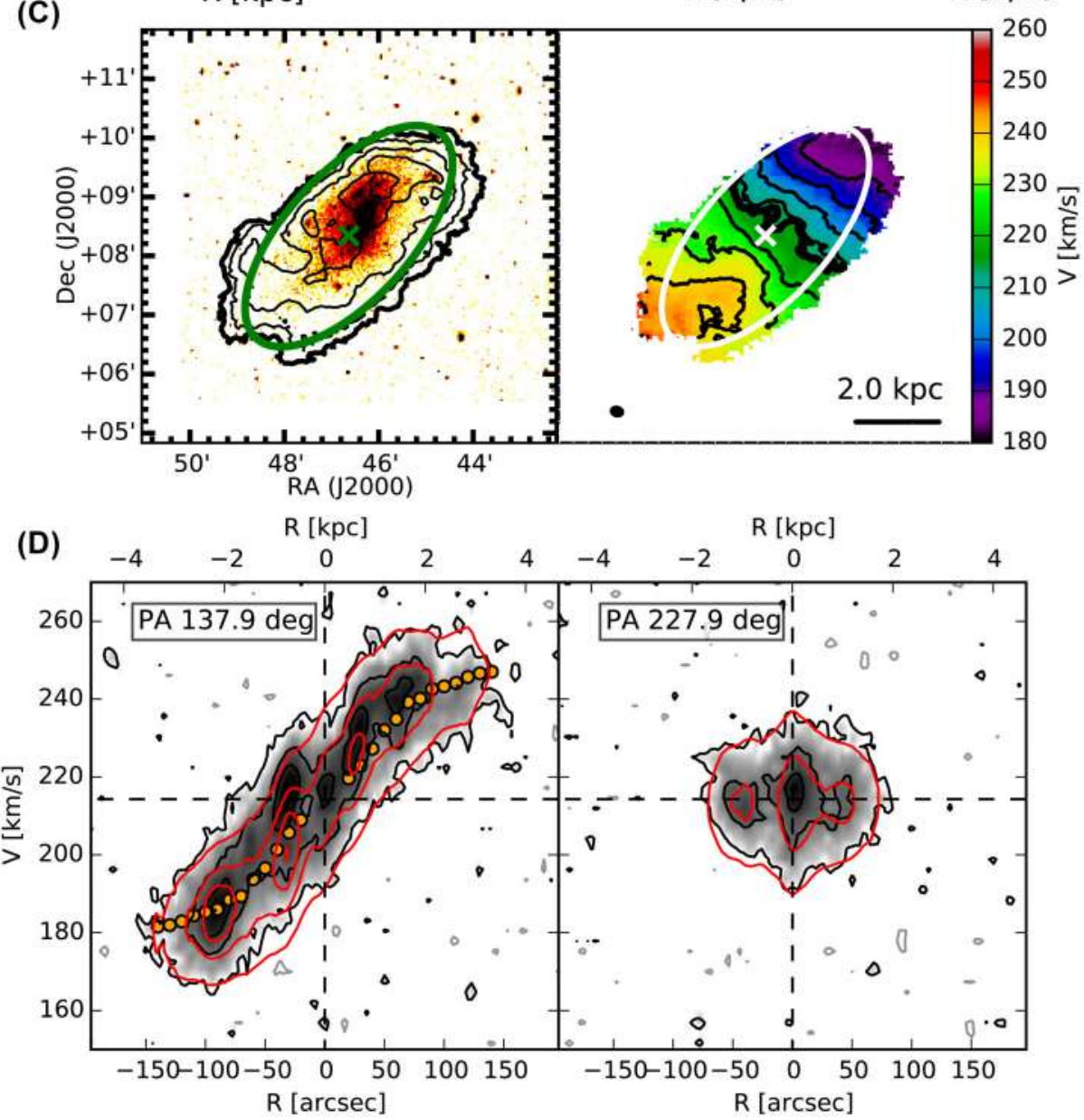

Figure 12. See caption in Appendix B. Notes - A: the empty circles indicates the region in which the circular velocity has been calculated as the mean between the approaching and receding rotation curves; $\mathrm{C}$ (left-hand panel): contours at $2^{n} \sigma_{3 \mathrm{~T}}$ and $\sigma_{3 \mathrm{~T}}=1.27 \mathrm{M}_{\odot} \mathrm{pc}^{-2}$ (thick contour), stellar map in SDSS $r$ band (Baillard et al. 2011); C (right-hand panel): contours at $V_{\text {sys }} \pm \Delta V$, where $\Delta V=8.0 \mathrm{~km} \mathrm{~s}^{-1}$ and $V_{\text {sys }}=214.3 \mathrm{~km} \mathrm{~s}^{-1}$ (thick contour); D: contours at $(2+6 n) \sigma_{\mathrm{ch}}$, where $\sigma_{\mathrm{ch}}=0.41 \mathrm{mJy} \mathrm{bm}^{-1}$, the grey contours are at $-2 \sigma_{\mathrm{ch}}$.

the $\mathrm{H}_{\mathrm{I}}$ data is very uncertain and we decided to set it to the optical value (Hunter \& Elmegreen 2006). The kinematics is dominated by the velocity dispersion; however, a weak velocity gradient is visible. The velocity gradient looks misaligned with both the stellar and the $\mathrm{H}_{\mathrm{I}}$ disc, so we set the initial values of $i$ and PA $\left(60^{\circ}\right.$ and $65^{\circ}$, respectively) using a by-eye inspection of the velocity field. This procedure is arbitrary, but it is important to note that the final circular-velocity curve and the related errors are independent of our 


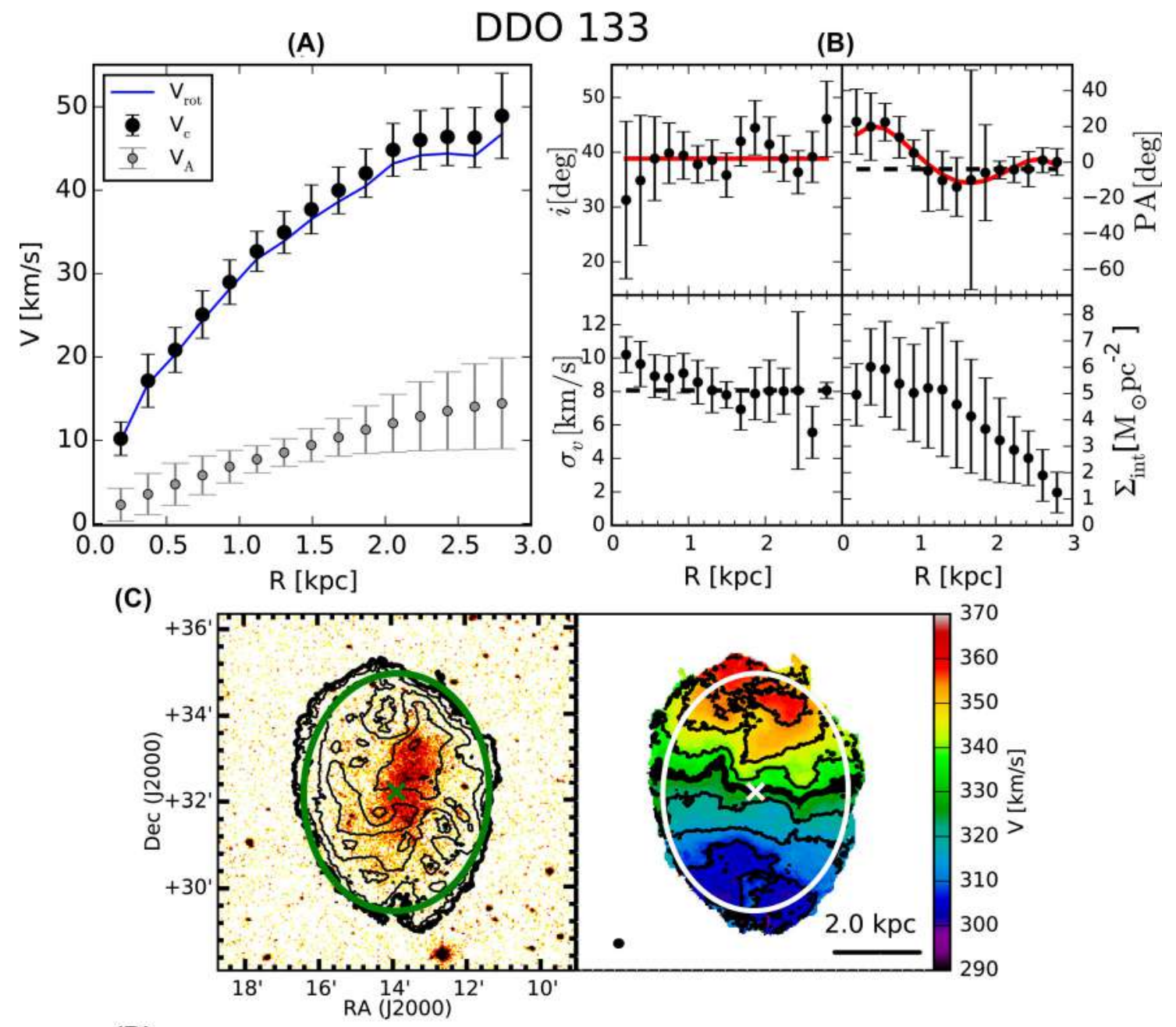

(D)

$\mathrm{R}[\mathrm{kpc}]$

$\mathrm{R}[\mathrm{kpc}]$

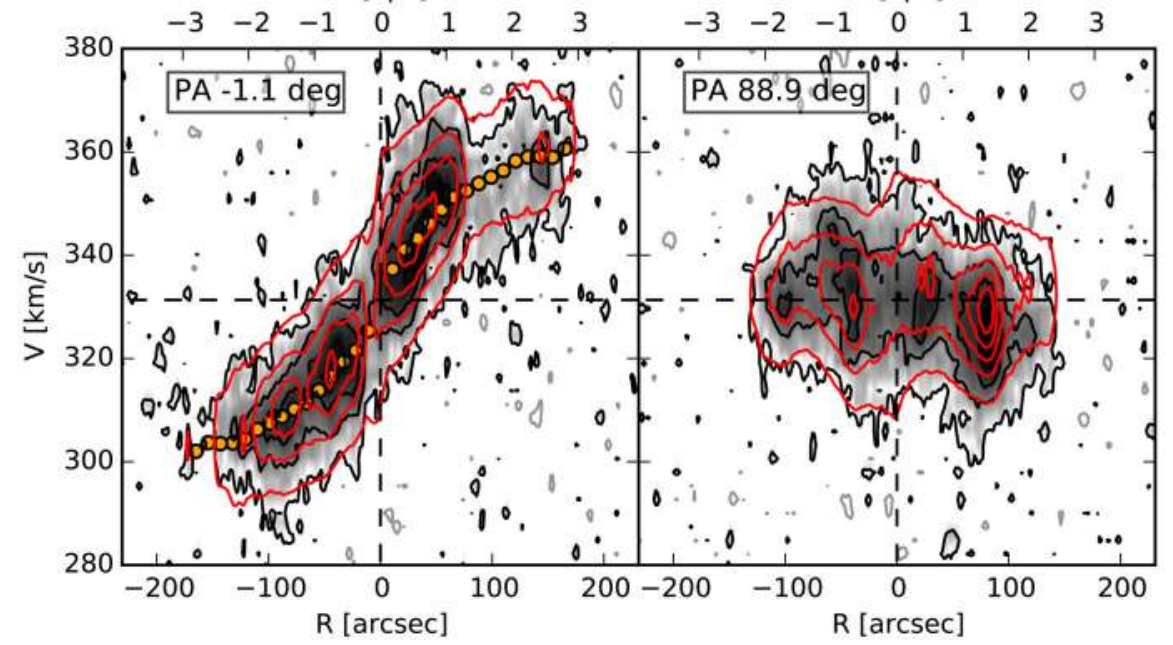

Figure 13. See caption in Appendix B. Notes - C (left-hand panel): contours at $2^{n} \sigma_{3 \mathrm{~T}}$ and $\sigma_{3 \mathrm{~T}}=0.89 \mathrm{M}_{\odot} \mathrm{pc}^{-2}$ (thick contour), stellar map in $R$ band from Cook et al. (2014); C (right-hand panel): contours at $V_{\text {sys }} \pm \Delta V$, where $\Delta V=8.0 \mathrm{~km} \mathrm{~s}^{-1}$ and $V_{\text {sys }}=331.3 \mathrm{~km} \mathrm{~s}^{-1}$ (thick contour); D: contours at (2+5n) $\sigma_{\text {ch }}$, where $\sigma_{\mathrm{ch}}=0.35 \mathrm{mJy} \mathrm{bm}^{-1}$, the grey contours are at $-2 \sigma_{\mathrm{ch}}$.

procedure since they are totally dominated by the asymmetric-drift correction (see Sections 4.3.1 and 4.3.4). The $V_{\text {rot }}$ found with 3DB in the first two rings (10 and 20 arcsec) is not well constrained, so we preferred to exclude them. Notice that along the minor axis there is an extended region with $\mathrm{H}_{\mathrm{I}}$ emission apparently not connected with the rotating disc. As in the case of DDO 53, this emission could trace an inflow/outflow.

(xiii) DDO 216. DDO 216 (also known as Pegasus dIrr, Fig. 17) is defined as a transitional dwarf galaxy (Cole et al. 1999). The galaxy shows a velocity gradient aligned with the $\mathrm{HI}_{\mathrm{I}}$ and the 


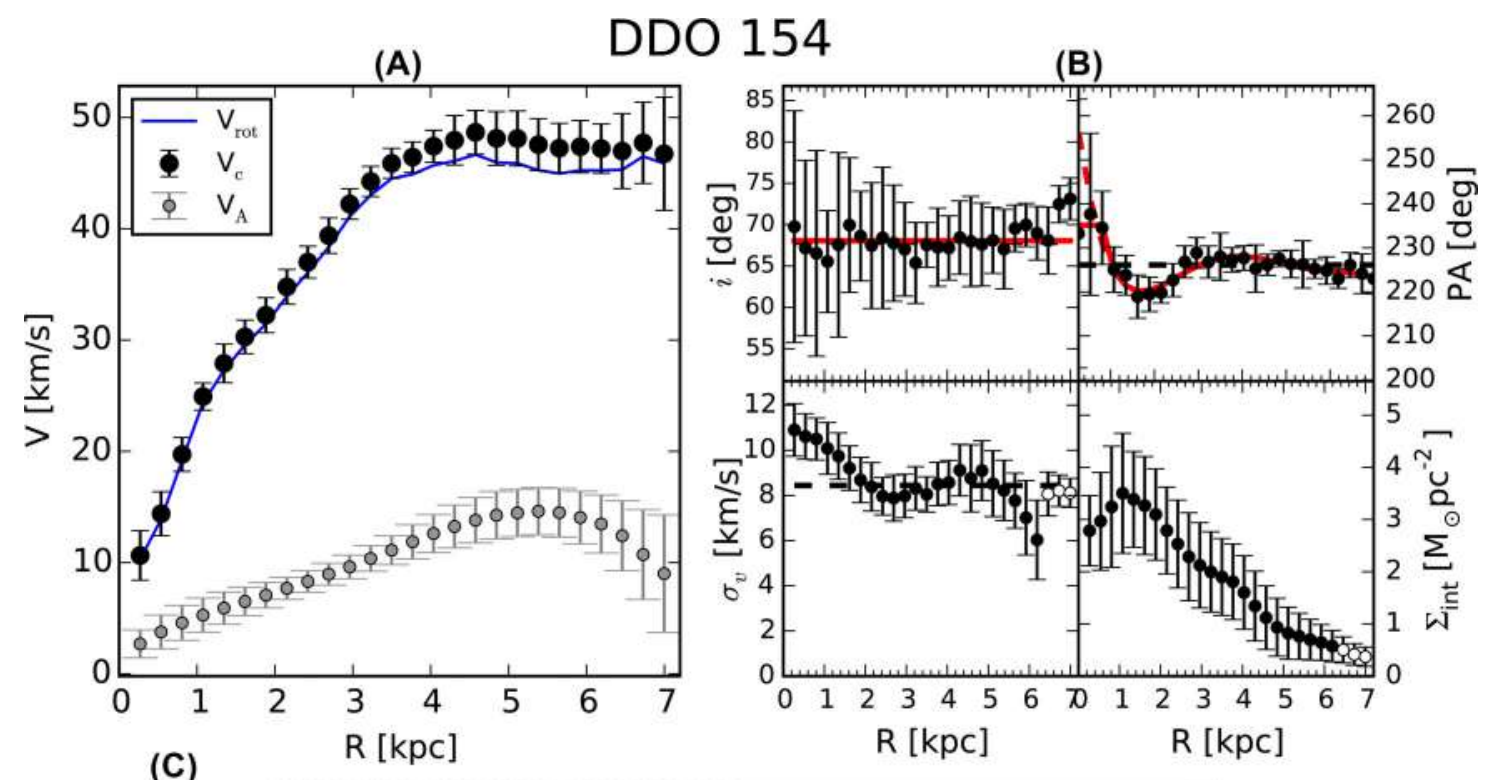

(C)
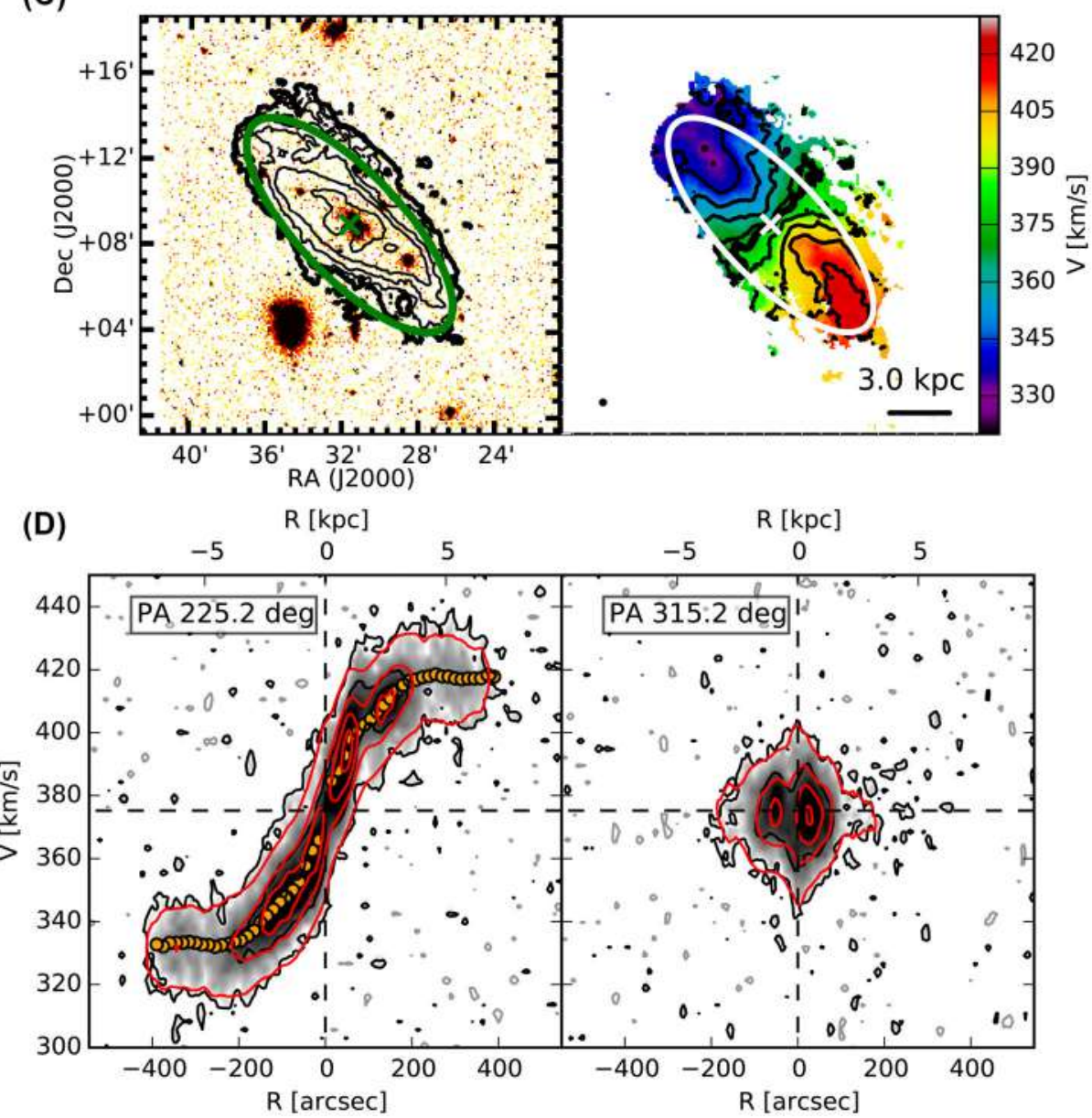

Figure 14. See caption in Appendix B. Notes - B (upper-left panel): the red dashed line shows the best-fitting polynomial to the data, the red continuous line shows the regularization of the radial profile used for this galaxy (see text for details); C (left-hand panel): contours at $2^{n} \sigma_{3 \mathrm{~T}}$ and $\sigma_{3 \mathrm{~T}}=0.52 \mathrm{M}_{\odot} \mathrm{pc}^{-2}$ (thick contour), stellar map in $R$ band from Cook et al. (2014); C (right-hand panel): contours at $V_{\text {sys }} \pm \Delta V$, where $\Delta V=10.0 \mathrm{~km} \mathrm{~s}^{-1}$ and $V_{\text {sys }}=375.2 \mathrm{~km} \mathrm{~s}^{-1}$ (thick contour); D: contours at $(2+8 n) \sigma_{\mathrm{ch}}$, where $\sigma_{\mathrm{ch}}=0.44 \mathrm{mJy} \mathrm{bm}^{-1}$, the grey contours are at $-2 \sigma_{\mathrm{ch}}$.

optical disc, but it could be entirely due to a single 'cloud' at a discrepant velocity in the approaching side of the galaxy (Stil \& Israel 2002a). For the purpose of our work, we assumed the gradient genuine and caused entirely by the gas rotation. The analysis of the alternative scenario can be found in Appendix C. Given the kinematic peculiarities (see Appendix C), we did not use 3DB to estimate $V_{\text {sys }}$ (Section 3.2.1). We tried different values between -186 and $-190 \mathrm{~km} \mathrm{~s}^{-1}$ and we decided to use $V_{\text {sys }}=-188 \mathrm{~km} \mathrm{~s}^{-1}$ 
(A)

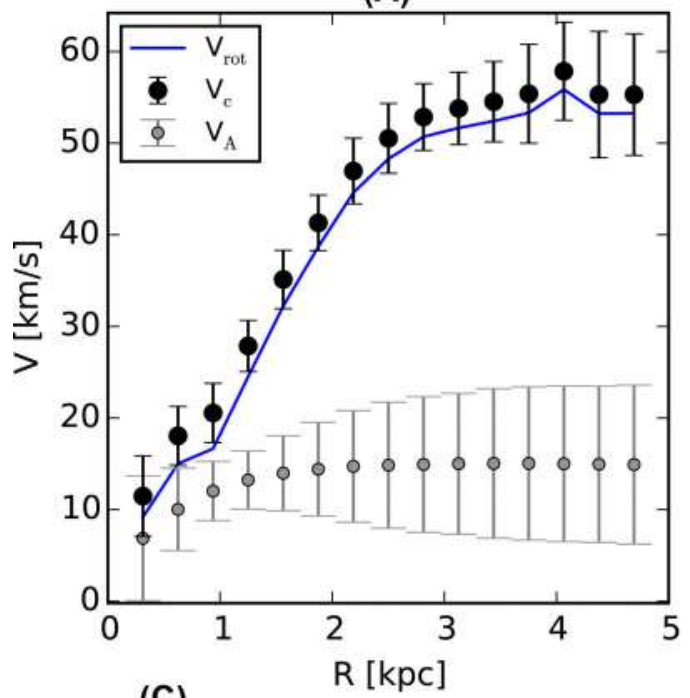

DDO 168

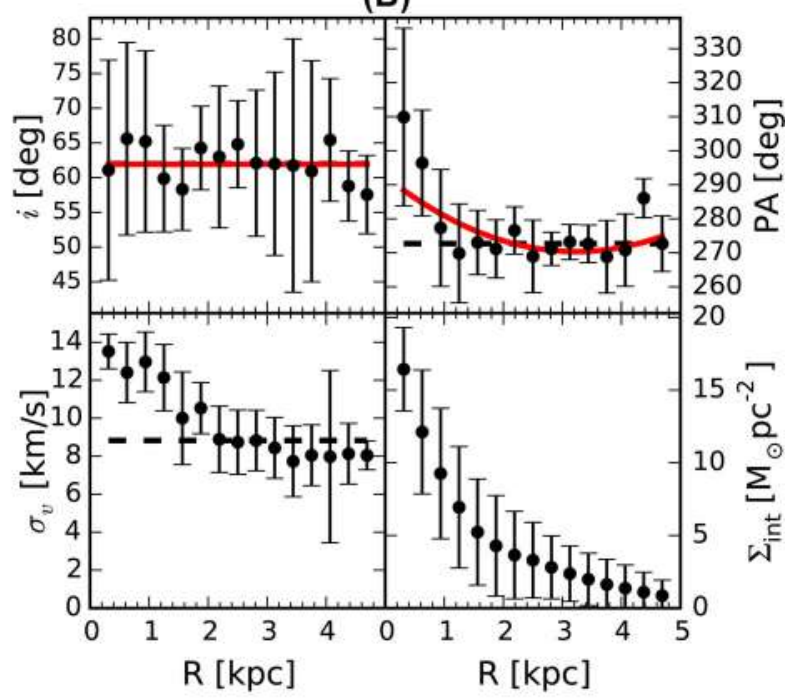

(C)
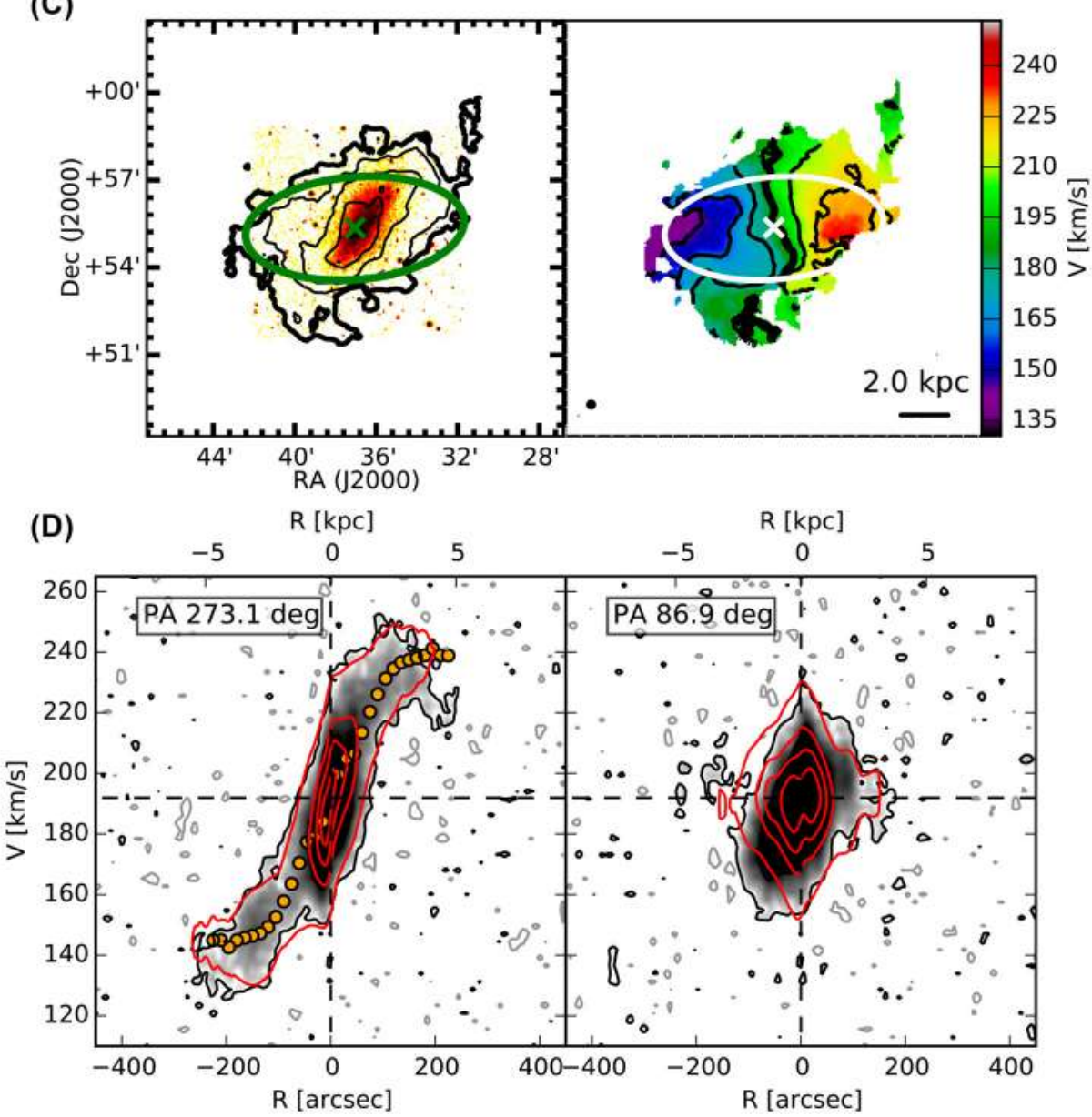

Figure 15. See caption in Appendix B. Notes - C (left-hand panel): contours at $3^{n} \sigma_{3 \mathrm{~T}}$ and $\sigma_{3 \mathrm{~T}}=0.54 \mathrm{M}_{\odot} \mathrm{pc}^{-2}$ (thick contour), stellar map in $r$ band from (Baillard et al. 2011); C (right-hand panel): contours at $V_{\text {sys }} \pm \Delta V$, where $\Delta V=15.0 \mathrm{~km} \mathrm{~s}^{-1}$ and $V_{\text {sys }}=191.9 \mathrm{~km} \mathrm{~s}^{-1}$ (thick contour); D: contours at $(2+15 n) \sigma_{\mathrm{ch}}$, where $\sigma_{\mathrm{ch}}=0.47 \mathrm{mJy} \mathrm{bm}^{-1}$, the grey contours are at $-2 \sigma_{\mathrm{ch}}$.

because this value minimizes the kinematic asymmetries between the receding and the approaching halves of the galaxy. We set the centre, $i_{\text {ini }}\left(65^{\circ}\right)$ and $\mathrm{PA}_{\text {ini }}\left(130^{\circ}\right)$ using the values obtained from the elliptical fit of the outermost $\mathrm{H}_{\mathrm{I}}$ contours $(R>600 \mathrm{pc})$. The best-fitting PA decreases from about $140^{\circ}$ to about $125^{\circ}$. (xiv) NGC 1569. NGC 1569 (Fig. 18) is a starburst galaxy with a very disturbed $\mathrm{H}_{\mathrm{I}}$ kinematics and morphology (Stil \& Israel 2002b; Johnson et al. 2012; Lelli et al. 2014b). We found that the best-fitting model shows a slight increase of $i$ and a slight decrease of PA as a function of radius. The best-fitting model is a good representation 
(A)

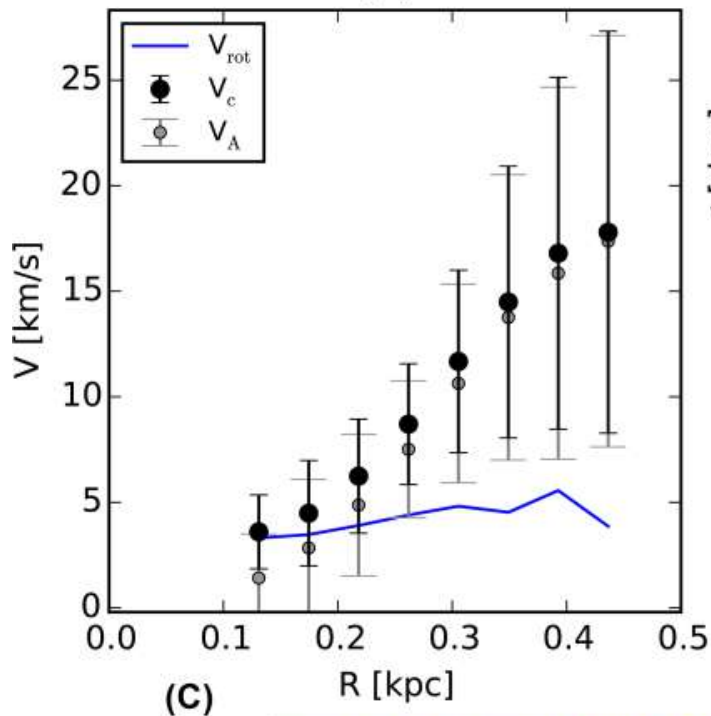

(C)
DDO 210

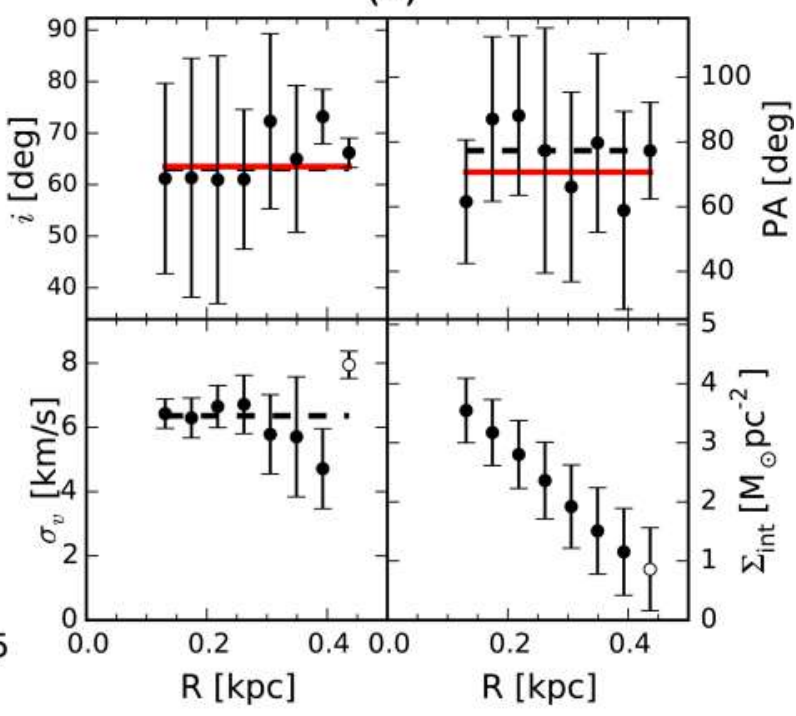

(B)
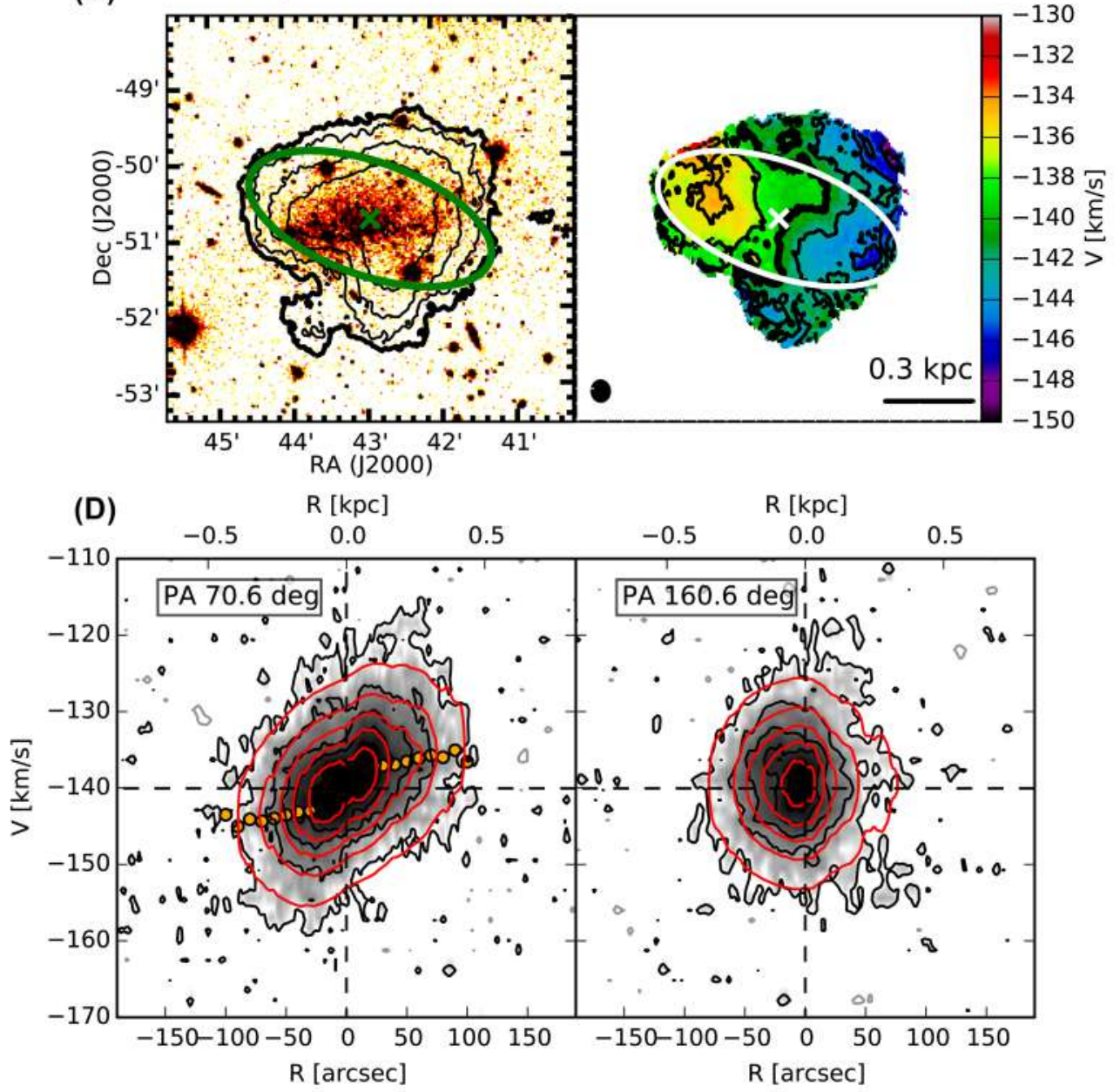

Figure 16. See caption in Appendix B. Notes - C (left-hand panel): contours at $2^{n} \sigma_{3 \mathrm{~T}}$ and $\sigma_{3 \mathrm{~T}}=0.59 \mathrm{M}_{\odot} \mathrm{pc}^{-2}$ (thick contour), stellar map in $R$ band from Cook et al. (2014); $\mathrm{C}$ (right-hand panel): contours at $V_{\text {sys }} \pm \Delta V$, where $\Delta V=2.5 \mathrm{~km} \mathrm{~s}^{-1}$ and $V_{\text {sys }}=-140.0 \mathrm{~km} \mathrm{~s}^{-1}$ (thick contour); D: contours at $(2+4 n) \sigma_{\mathrm{ch}}$, where $\sigma_{\mathrm{ch}}=0.75 \mathrm{mJy} \mathrm{bm}^{-1}$, the grey contours are at $-2 \sigma_{\mathrm{ch}}$. 


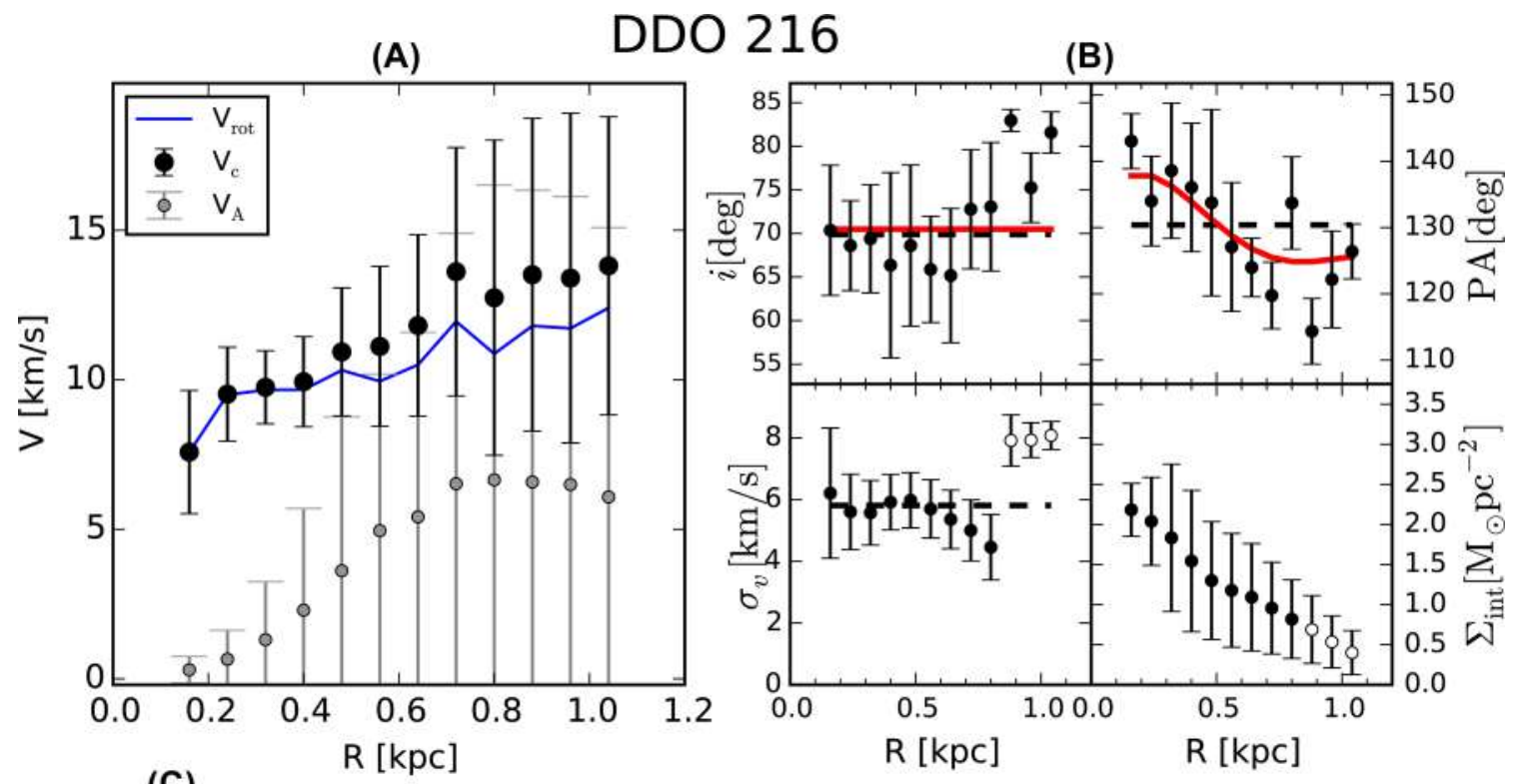

(C)
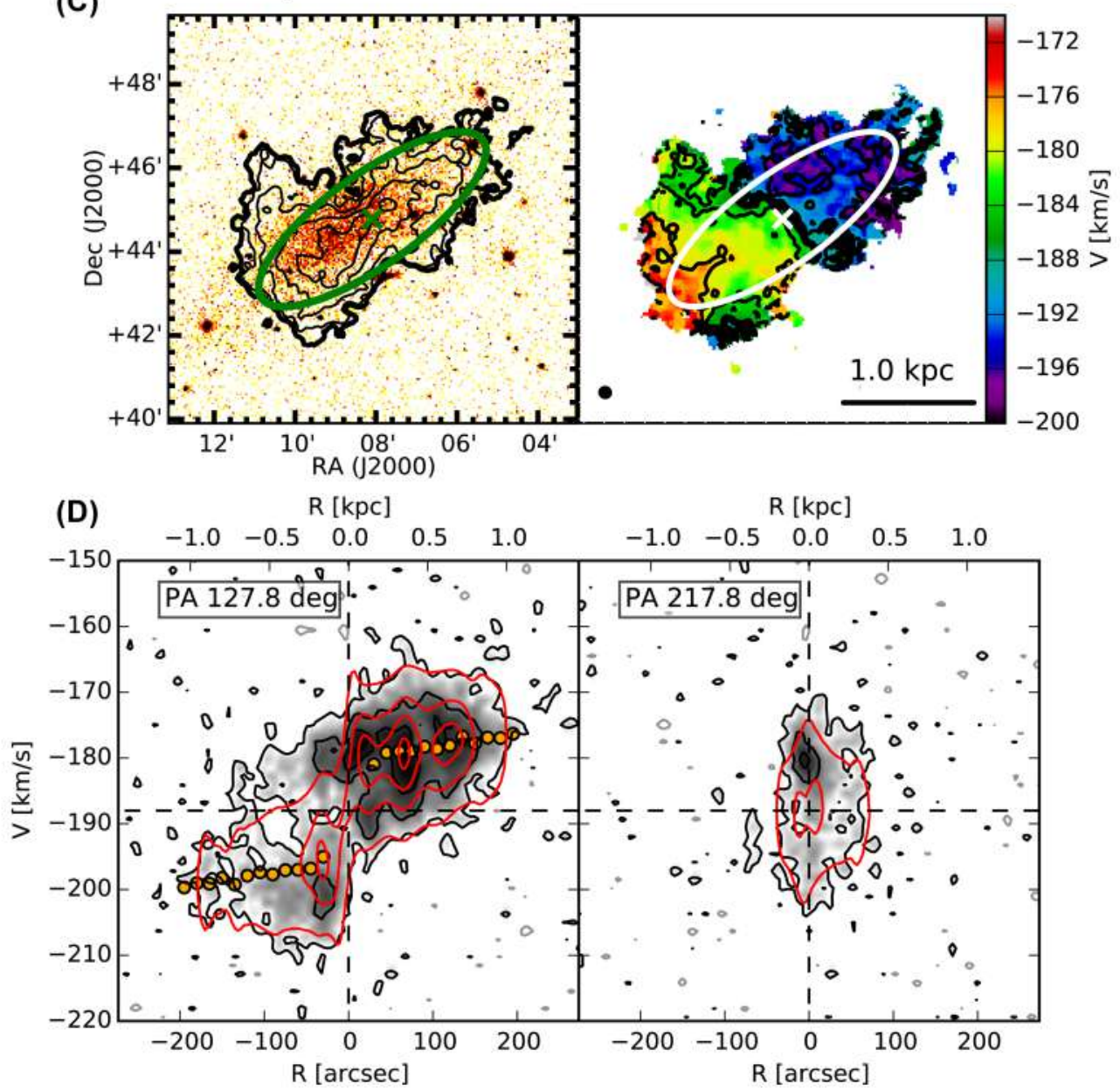

Figure 17. See caption in Appendix B. Notes - C (left-hand panel): contours at $2^{n} \sigma_{3 \mathrm{~T}}$ and $\sigma_{3 \mathrm{~T}}=0.40 \mathrm{M}_{\odot} \mathrm{pc}^{-2}$ (thick contour), stellar map in SDSS $r$ band (Knapen et al. 2014); C (right-hand panel): contours at $V_{\text {sys }} \pm \Delta V$, where $\Delta V=5.0 \mathrm{~km} \mathrm{~s}^{-1}$ and $V_{\text {sys }}=-188.0 \mathrm{~km} \mathrm{~s}^{-1}$ (thick contour); D: contours at $(2+6 n) \sigma_{\mathrm{ch}}$, where $\sigma_{\mathrm{ch}}=0.91 \mathrm{mJy} \mathrm{bm}^{-1}$, the grey contours are at $-2 \sigma_{\mathrm{ch}}$.

of the large-scale structure and kinematics of the H I disc, but it fails to reproduce the small-scale local features. The interstellar medium of this galaxy is highly turbulent: the velocity dispersion found with $3 \mathrm{DB}$ is about $20 \mathrm{~km} \mathrm{~s}^{-1}$ and the asymmetric-drift correction dominates at all radii. For this reason, the kinematic data reported here should be used with caution especially at the inner radii (empty circles in Box A in Fig. 18) where no significant rotation of the gas is observed (see also Lelli et al. 2014b). 


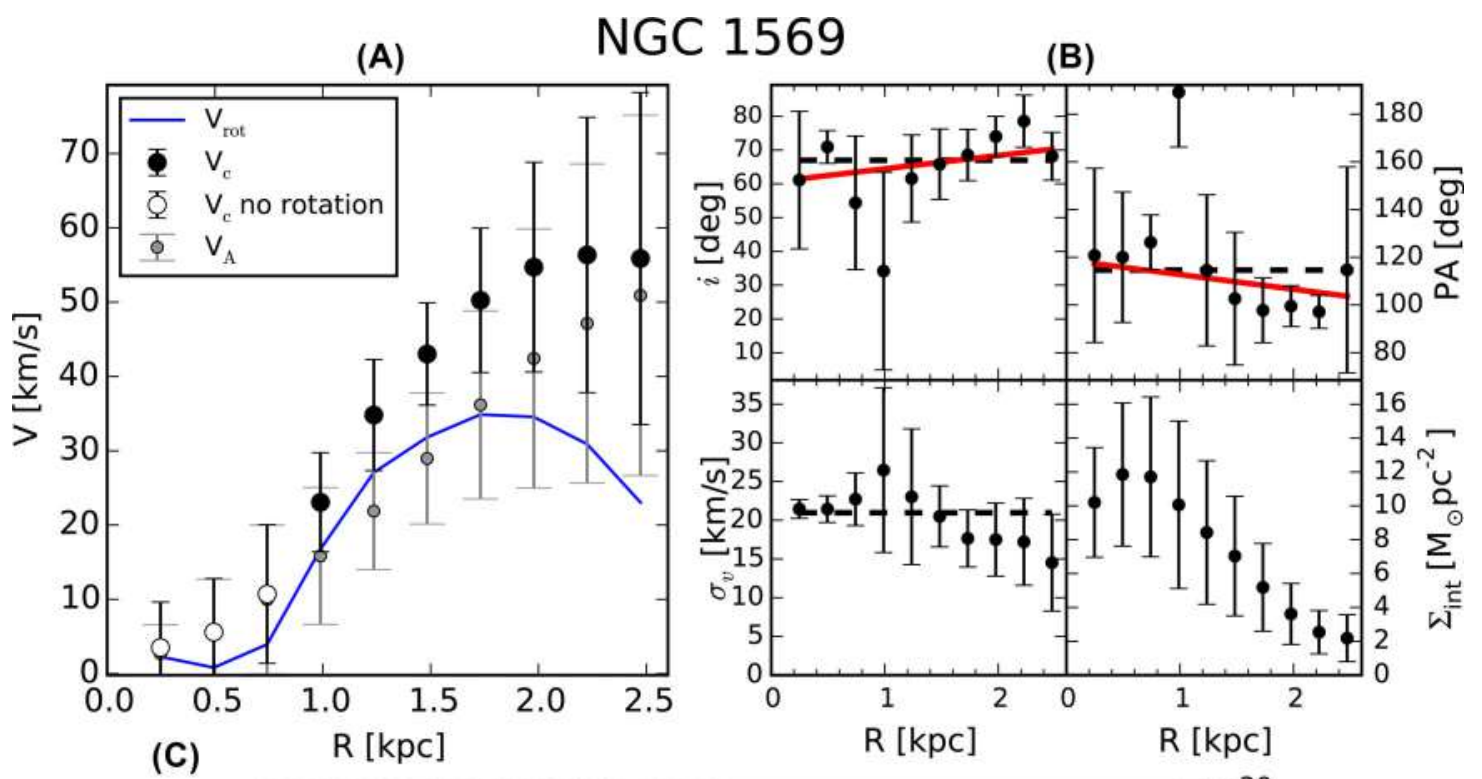

(C)
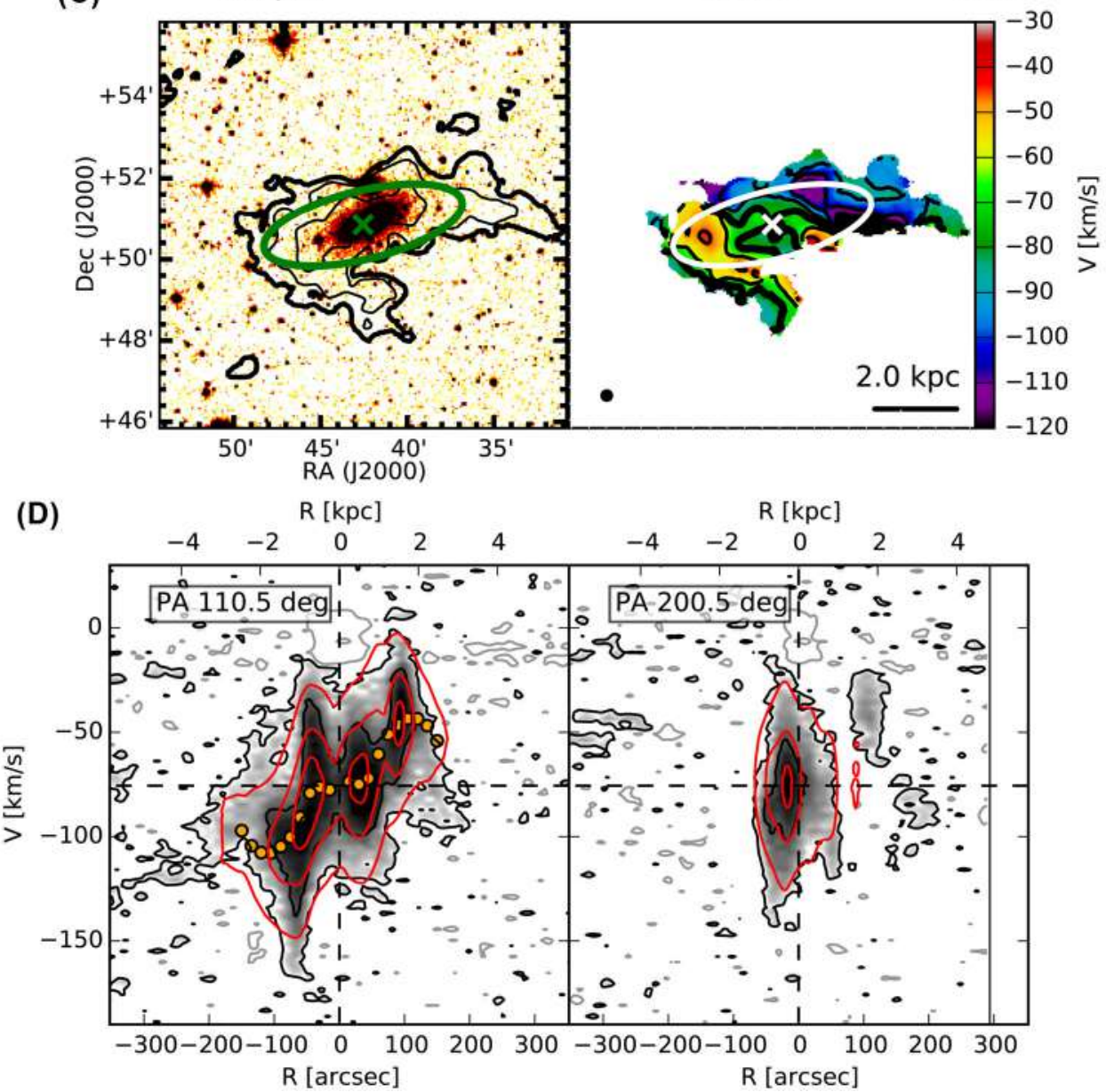

Figure 18. See caption in Appendix B. Notes - A: the empty circles indicate the region in which there are no significant signs of gas rotation; C (left-hand panel): contours at $4^{n} \sigma_{3 \mathrm{~T}}$ and $\sigma_{3 \mathrm{~T}}=1.10 \mathrm{M}_{\odot} \mathrm{pc}^{-2}$ (thick contour), stellar map in $J$ band from Jarrett et al. (2003); C (right-hand panel): contours at $V_{\text {sys }} \pm$ $\Delta V$, where $\Delta V=15.0 \mathrm{~km} \mathrm{~s}^{-1}$ and $V_{\text {sys }}=-75.6 \mathrm{~km} \mathrm{~s}^{-1}$ (thick contour); D: contours at $(2+8 n) \sigma_{\mathrm{ch}}$, where $\sigma_{\mathrm{ch}}=0.77 \mathrm{mJy}^{-1}$, the grey contours are at $-2 \sigma_{\mathrm{ch}}$.

(xv) NGC 2366. The HI disc of NGC 2366 (Fig. 19) is quite regular, although it shows some peculiar features. The Hi emission on the channel maps (Fig. 4) indicates the presence of two ridges located in the north-west and south-east (less prominent) of the disc running parallel to the major axis. The ridges show different kinematics with respect to the disc (see Oh et al. 2008) and their origin is not clear (see Hunter, Elmegreen \& van Woerden 2001 for a detailed discussion). We checked that 3DB was not affected by the 


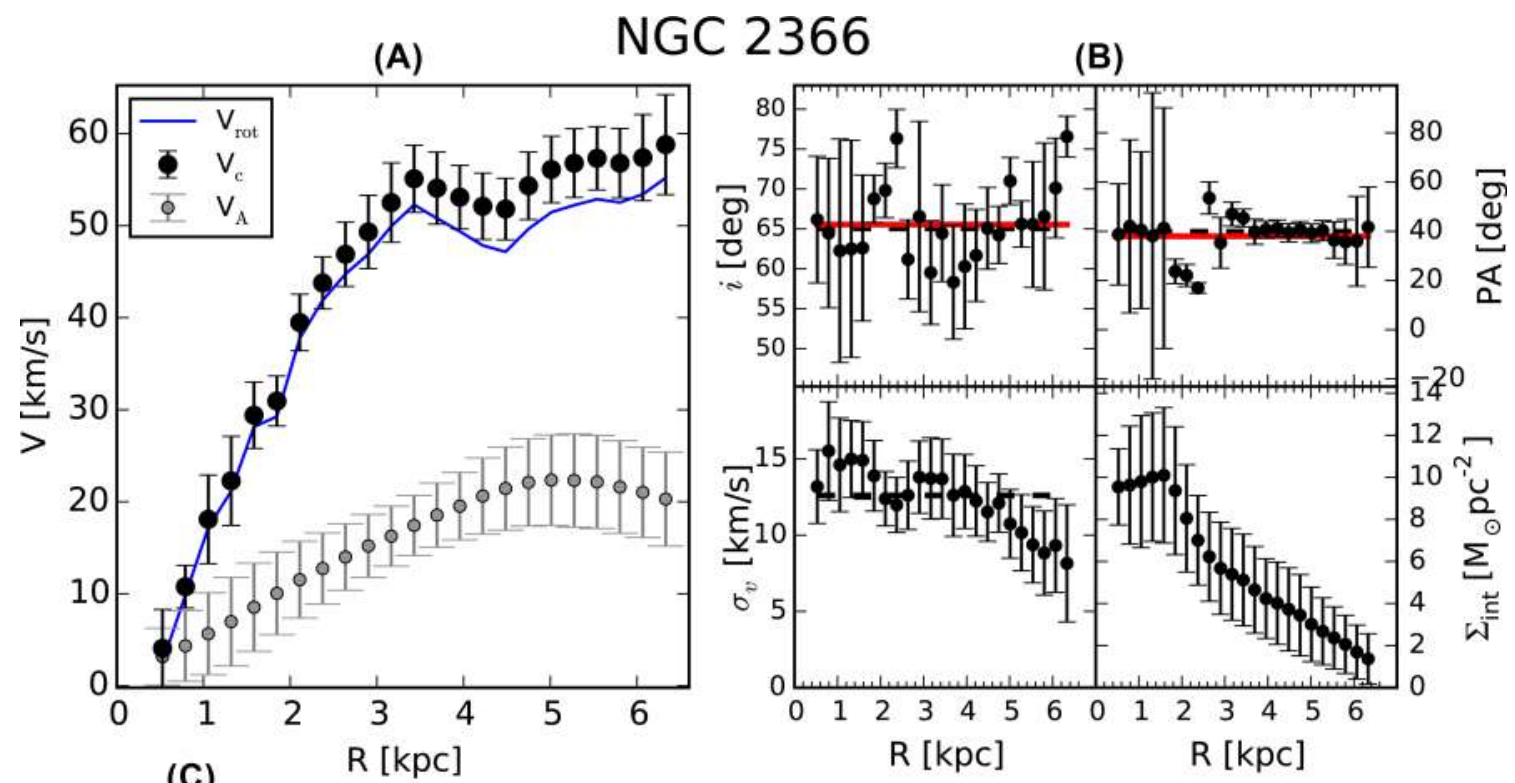

(C)
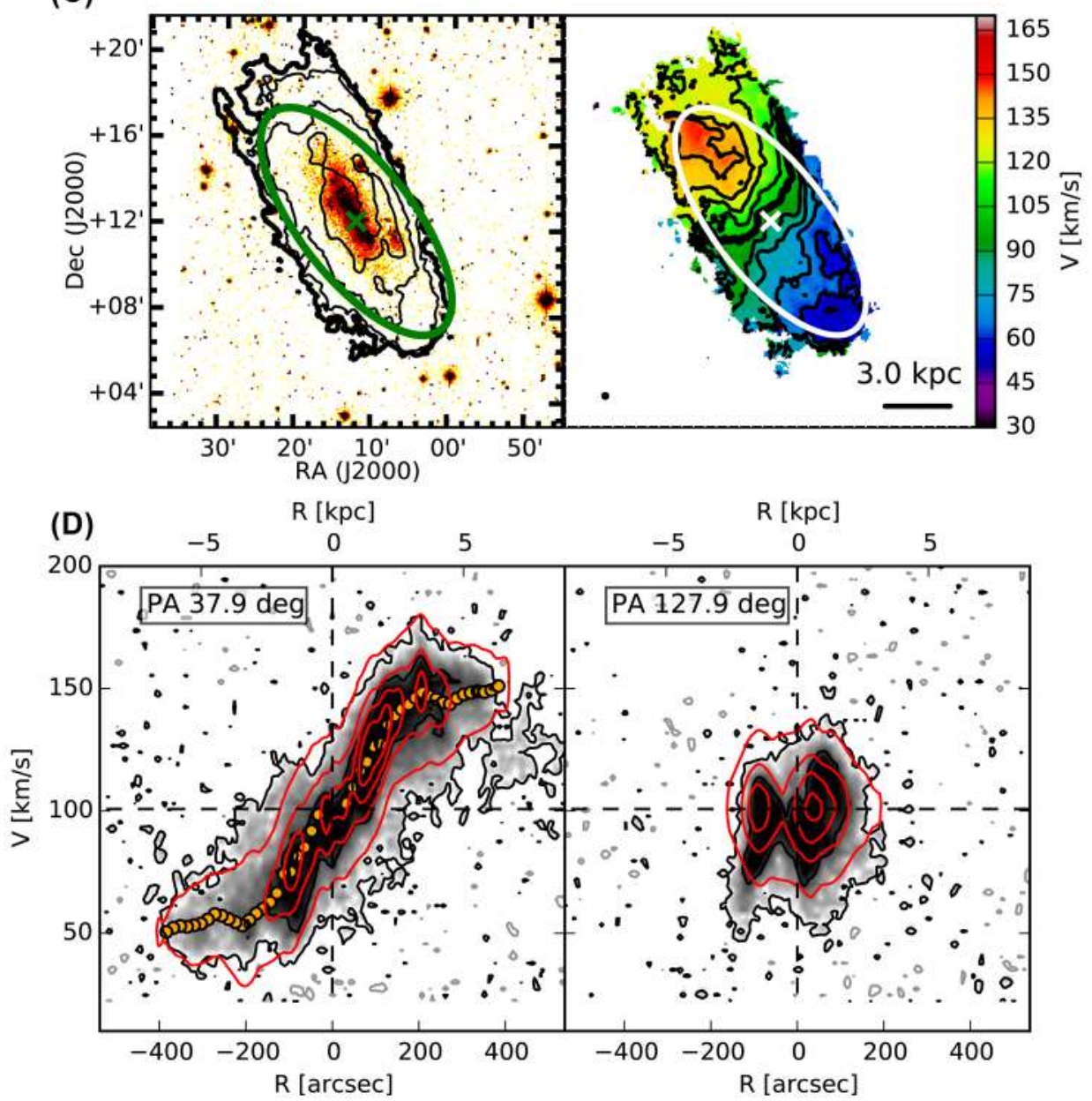

Figure 19. See caption in Appendix B. Notes $-\mathrm{C}$ (left-hand panel): contours at $3^{n} \sigma_{3 \mathrm{~T}}$ and $\sigma_{3 \mathrm{~T}}=0.59 \mathrm{M}_{\odot} \mathrm{pc}^{-2}$ (thick contour), stellar map in $R$ band from Cook et al. (2014); $\mathrm{C}$ (right-hand panel): Contours at $V_{\text {sys }} \pm \Delta V$, where $\Delta V=10.0 \mathrm{~km} \mathrm{~s}^{-1}$ and $V_{\text {sys }}=100.8 \mathrm{~km} \mathrm{~s}^{-1}$ (thick contour); D: contours at $(2+10 n) \sigma_{\mathrm{ch}}$, where $\sigma_{\mathrm{ch}}=0.52 \mathrm{mJy} \mathrm{bm}^{-1}$, the grey contours are at $-2 \sigma_{\mathrm{ch}}$.

presence of this feature. From the PV along the major axis (Panel D in Fig. 19), it is clear that the 3DB model does not reproduce some emission close to the systemic velocity, especially in the receding side where the gas is seen also at 'forbidden' velocities below $V_{\text {sys }}$.
As already stated by Lelli et al. (2014b), this is probably due to the presence of some extraplanar gas that is rotating at lower velocity with respect to the gas in the disc (see e.g. Fraternali et al. 2002). As in Lelli et al. (2014b) and in O15, we do not find that the rotation 


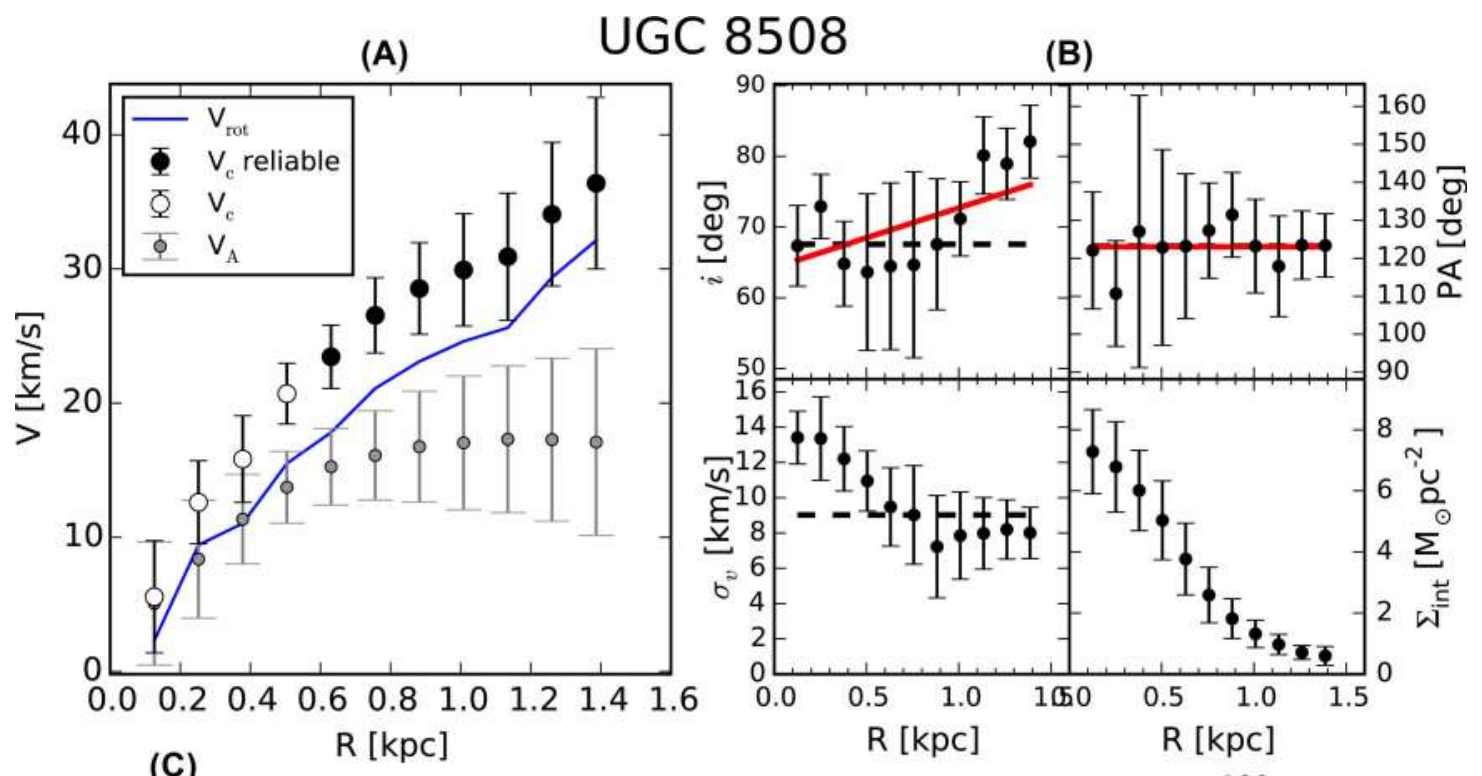

(C)
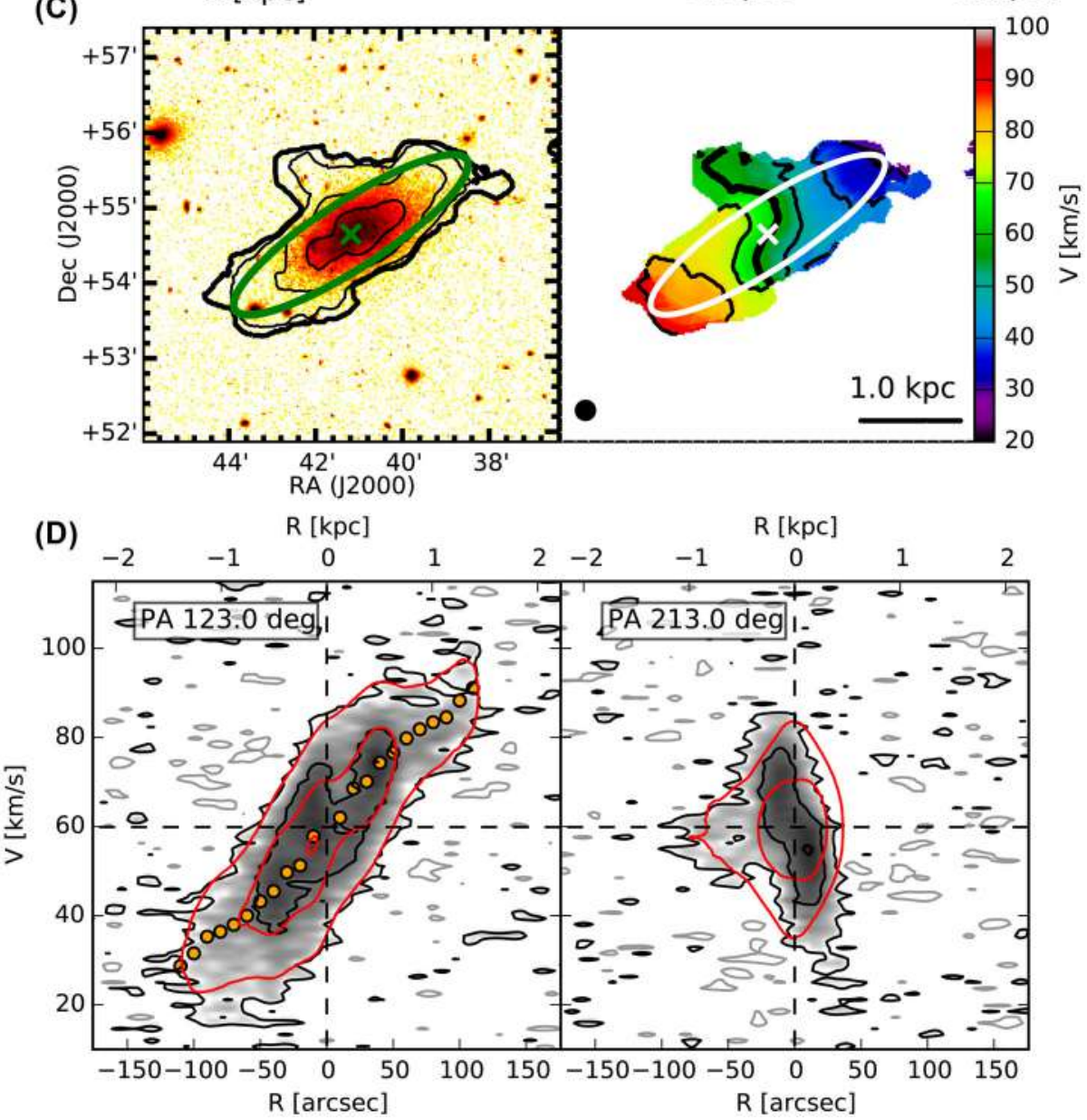

Figure 20. See caption in Appendix B. Notes - A: the empty circles indicate the region of the galaxy with peculiar kinematics (see the text); C (left-hand panel): contours at $3^{n} \sigma_{3 \mathrm{~T}}$ and $\sigma_{3 \mathrm{~T}}=0.54 \mathrm{M}_{\odot} \mathrm{pc}^{-2}$ (thick contour), stellar map in $R$ band from Cook et al. (2014); $\mathrm{C}$ (right-hand panel): contours at $V_{\text {sys }} \pm$ $\Delta V$, where $\Delta V=10.0 \mathrm{~km} \mathrm{~s}^{-1}$ and $V_{\mathrm{sys}}=59.9 \mathrm{~km} \mathrm{~s}^{-1}$ (thick contour); D: contours at $(2+6 n) \sigma_{\mathrm{ch}}$, where $\sigma_{\mathrm{ch}}=1.31 \mathrm{mJy} \mathrm{bm}^{-1}$, the grey contours are at $-2 \sigma_{\mathrm{ch}}$.

curve declines beyond $5 \mathrm{kpc}$, as instead claimed by both Hunter et al. (2001) and van Eymeren et al. (2009a).

(xvi) UGC 8508. The $\mathrm{H}_{\mathrm{I}}$ and the stellar disc of UGC 8508 (Fig. 20) are aligned but the analysis of the HI map favours an $i$ slightly higher than the value obtained from the stellar disc (Hunter \& Elmegreen 2006). We found that the data cube is better reproduced with a linearly increasing $i$. Notice that in the inner part of the galaxy $(R<0.6 \mathrm{kpc})$ the kinematics is very peculiar 
as it is visible by the S-shaped iso-velocity contours (right-hand panel $\mathrm{C}$ in Fig. 20). This kind of distortions can be related to an abrupt variation of the PA and/or to the presence of radial motions (Fraternali et al. 2001) as well as to a deviation from axisymmetry of the galactic potential (Swaters et al. 1999). We tested the hypothesis of a radially varying PA and the presence of non-zero radial velocities $\left(V_{\mathrm{rad}}\right)$ performing a 2D analysis of the velocity field with ROTCUR (Begeman 1987). We found that the combination of the two effects can partially explain the distortions of the velocity field, but their magnitude is too large to be physically plausible. Fortunately, the final rotation curves obtained including radial motions and/or the varying PA are compatibles with the results we found with $3 \mathrm{DB}$, though the inner points (empty circles in Box A in Fig. 20) should be treated with caution.

(xvii) WLM. The HI and the optical discs of WLM (Fig. 21) are well aligned, but the best-fitting $i$ looks slightly too edge-on with respect to the $\mathrm{H}$ I contours (see ellipses in Fig. 21). The excess of the emission around the minor axis could be partially due to the thickness of the gaseous layer (Section 7.1, see also Leaman et al. 2012). Further details on the analysis of WLM can be found in Read et al. (2016c).

\section{APPLICATION: TEST OF THE BARYONIC TULLY-FISHER RELATION}

The BTFR links a characteristic circular velocity $(V)$ of a galaxy with its total baryonic mass $\left(M_{\mathrm{bar}}\right)$. The relation, in the logarithmic form

$\log \left(\frac{M_{\mathrm{bar}}}{\mathrm{M}_{\odot}}\right)=s \log \left(\frac{V}{\mathrm{~km} \mathrm{~s}^{-1}}\right)+A$,

is very tight and extends over six decades in $M_{\text {bar }}$ (McGaugh 2012; Lelli et al. 2016). The existence of this relation represents a fundamental benchmark for cosmological models and for galaxy formation theories (McGaugh et al. 2000; Brook et al. 2016; Di Cintio \& Lelli 2016). In this context, it is very important to extend the study of the BTFR down to extremely low-mass dwarf galaxies (e.g. Begum et al. 2008). In this section, we present the BTFR for the galaxies in our sample (Fig. 22) and then we compare it with the results of Lelli et al. (2016, Fig. 23). The derivation of the baryonic mass and of the characteristic velocity is described below.

(i) Rotation velocity. The rotation velocities used in the TullyFisher relation (TFR) are $W_{20}$ and $V_{\text {flat }}: W_{20}$ is the velocity width of the global $\mathrm{H}_{\text {I }}$ line profiles, while $V_{\text {flat }}$ is the value of the flat portion of the rotation curve. Verheijen (2001) found that $V_{\text {flat }}$ minimizes the scatter in the TFR, so it is the best way to study the BTFR using highresolution $\mathrm{H}$ I data (see also Brook et al. 2016). However, several rotation curves of dIrrs do not reach the flat part (e.g. DDO 53, Fig. 9) or the flattening is entirely due to the asymmetric-drift correction (e.g. NGC 1569, Fig. 18). As indicator of the rotation velocity, we therefore used the velocity of the outer $\operatorname{disc}\left(V_{\mathrm{o}}\right)$ defined as the mean circular velocity of the last three fitted rings (see Section 4.2). $V_{\mathrm{o}}$ is a measure of $V_{\text {flat }}$ for galaxies in which the flat part of the rotation curve is observed. For galaxies with a rising rotation curve, $V_{\mathrm{o}}$ is an estimate of the maximum circular velocity within the considered radial range. Galaxies with rising rotation curves are indicated with empty markers in Figs 22 and 23 . The error on $V_{\mathrm{o}}$ is conservatively assumed as the maximum error between the three values used to calculate $V_{\mathrm{o}}$. In the five galaxies with a low $i$ (squares in Figs 22 and 23), the uncertainties on $i$ and on the velocities can be largely underestimated (see Section 5 and Table 2), so we present these galaxies with a bar that indicates the interval between a minimum (assuming $i=40^{\circ}$ ) and a maximum (assuming $i=20^{\circ}$ ) value of $V_{\mathrm{o}}$ (see equation 1 ).

(ii) Baryonic mass. The total baryonic mass of the galaxies has been calculated as

$M_{\mathrm{bar}}=M_{*}+1.33 M_{\mathrm{HI}}$,

where $M_{*}$ is the stellar mass, $M_{\mathrm{HI}}$ is the mass of the atomic hydrogen and the factor 1.33 takes into account the presence of Helium (Begum et al. 2008; Lelli et al. 2016). The molecular gas is likely irrelevant in the mass budget of dwarf galaxies (Taylor, Kobulnicky \& Skillman 1998). The mass of atomic gas is measured from the H I data cubes (Section 4.1.1 and Table 1), while the stellar masses are from Walter \& Brinks (2001) for DDO 47 and from Zhang et al. (2012) for all the other galaxies. The estimate of the mass is proportional to the square of the galactic distance, therefore errors on the distance add further uncertainties on the final estimate of the baryonic mass. Unfortunately, the works we used to take the stellar masses (Walter \& Brinks 2001; Zhang et al. 2012) and the distances (Hunter et al. 2012) do not report the errors on their measures, so we assumed a conservative error of the 30 per cent for $M_{\mathrm{bar}}$. We also performed a deeper analysis for the distance uncertainties. The relative difference between the masses estimated assuming two different distances is

$\delta_{\mathrm{D}}=\frac{M\left(\mathrm{D}_{1}\right)-\mathrm{M}\left(\mathrm{D}_{2}\right)}{\mathrm{M}\left(\mathrm{D}_{1}\right)}=\frac{\mathrm{D}_{1}^{2}-\mathrm{D}_{2}^{2}}{\mathrm{D}_{1}^{2}}$.

For each galaxy in our sample, we choose the best distance estimator $^{4}$ available on NASA/IPAC Extragalactic Database (NED) and we considered the minimum $\left(D_{\mathrm{NED}}^{\min }\right)$ and the maximum $\left(\mathrm{D}_{\mathrm{NED}}^{\max }\right)$ estimate of the distance, then using equation (15) we calculated

$\delta_{\mathrm{D}}^{\min / \max }=1-\left(\frac{\min \left(\mathrm{D}, \mathrm{D}_{\mathrm{NED}}^{\min / \max }\right)}{\max \left(\mathrm{D}, \mathrm{D}_{\mathrm{NED}}^{\min / \max }\right)}\right)^{2}$,

where $D$ is the distance assumed in this work (see Table 1 ). When $\delta_{\mathrm{D}}$ is large the error on the total mass is dominated by the uncertainty on the distance. Three galaxies have $\delta_{\mathrm{D}}$ larger than the 60 per cent: DDO $47\left(\delta_{\mathrm{D}}^{\max }=62\right.$ per cent $)$, DDO $101\left(\delta_{\mathrm{D}}^{\max }=85\right.$ per cent $)$ and NGC $1569\left(\delta_{\mathrm{D}}^{\min }=68\right.$ per cent $)$. For these galaxies, we do not show the $1 \sigma$ error on the baryonic mass, but a magenta bar indicating the interval of mass found assuming the distance $D$ or the distance $D_{\mathrm{NED}}^{\min / \max }$.

In Fig. 23, we compare our data with a recent fit to the BTFR (Lelli et al. 2016). Interestingly our data overlap between $10^{8}$ and $10^{9} \mathrm{M}_{\odot}$. In this range, our data are perfectly compatible with the parameters of the BTFR estimated in Lelli et al. (2016) $(s=3.95$ and $A=1.86$ in equation 13 ); moreover, we have fitted our data with a linear relation leaving the intrinsic scatter $(f)$ as the only free parameter (the other parameters have been fixed to the values found by Lelli et al. 2016). The best-fitting result is $f=0.1 \pm 0.1$, so we also confirm the very small scatter around the relation in contrast to Begum et al. (2008). This remains the case, even when including galaxies with rising rotation curves: the only two outliers are DDO 50 (a nearly face-on galaxy) and DDO 101 (for which the uncertainty on the distance is large; Section 5, see also Read et al. 2016c). Below $10^{8} \mathrm{M}_{\odot}$ the distribution of the galaxies looks again compatible with the relation of Lelli et al. (2016). It appears

\footnotetext{
${ }^{4}$ The scale of distance estimators is, from the best to the worst: Cepheids, RGB-Tip, CMD, Brightest-Stars, Tully-Fisher relation.
} 

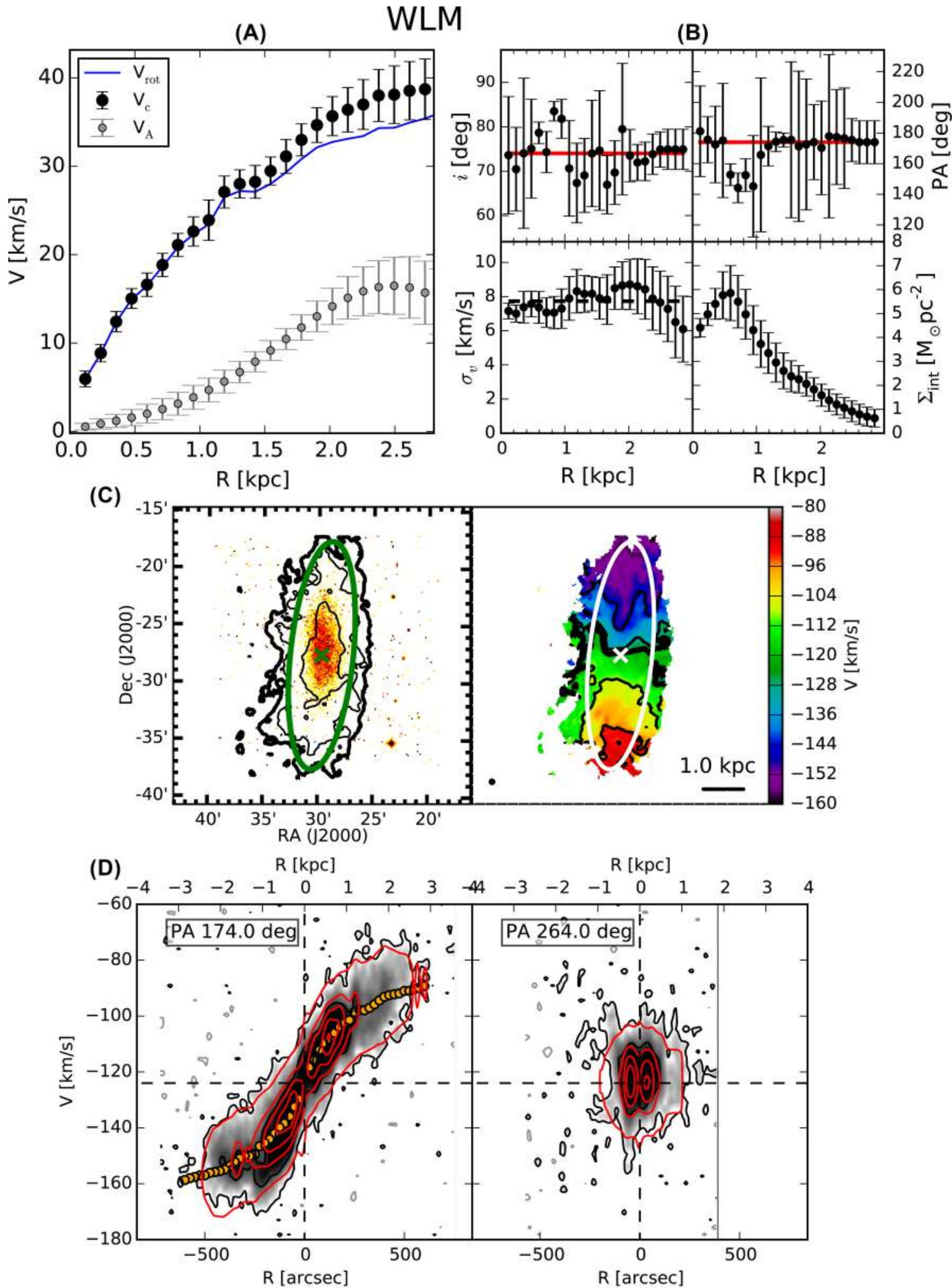

Figure 21. See caption in Appendix B. Notes $-\mathrm{C}$ (left-hand panel): contours at $2^{n} \sigma_{3 \mathrm{~T}}$ and $\sigma_{3 \mathrm{~T}}=0.58 \mathrm{M}_{\odot} \mathrm{pc}^{-2}$ (thick contour), stellar map in $R$ band from Cook et al. (2014); $\mathrm{C}$ (right-hand panel): contours at $V_{\mathrm{sys}} \pm \Delta V$, where $\Delta V=20.0 \mathrm{~km} \mathrm{~s}^{-1}$ and $V_{\mathrm{sys}}=-124.0 \mathrm{~km} \mathrm{~s}^{-1}$ (thick contour); D: contours at $(2+4 n) \sigma_{\mathrm{ch}}$, where $\sigma_{\mathrm{ch}}=2.00 \mathrm{mJy} \mathrm{bm}^{-1}$, the grey contours are at $-2 \sigma_{\mathrm{ch}}$. 


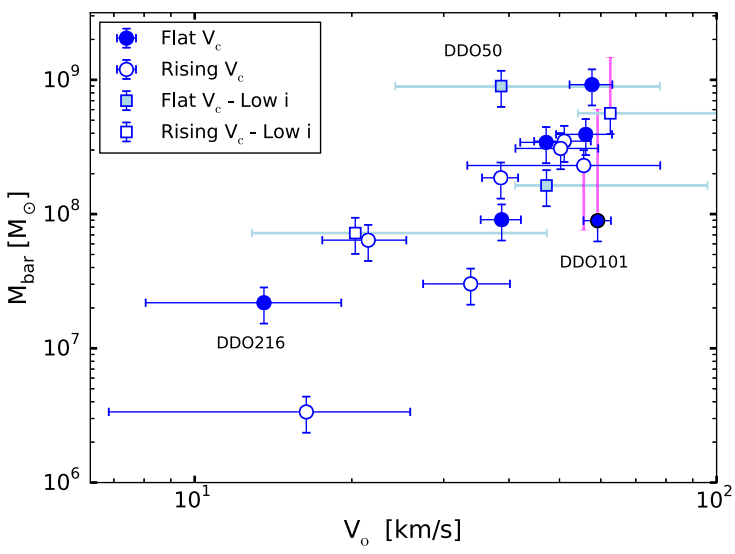

Figure 22. BTFR for the galaxies in our sample. The empty and the solid symbols indicate galaxies with rising or flat rotation curve, respectively. The squares indicate nearly face-on galaxies. The blue bars indicate $1 \sigma$ errors. The light-blue and the magenta bars indicate intervals of allowed values (see text).

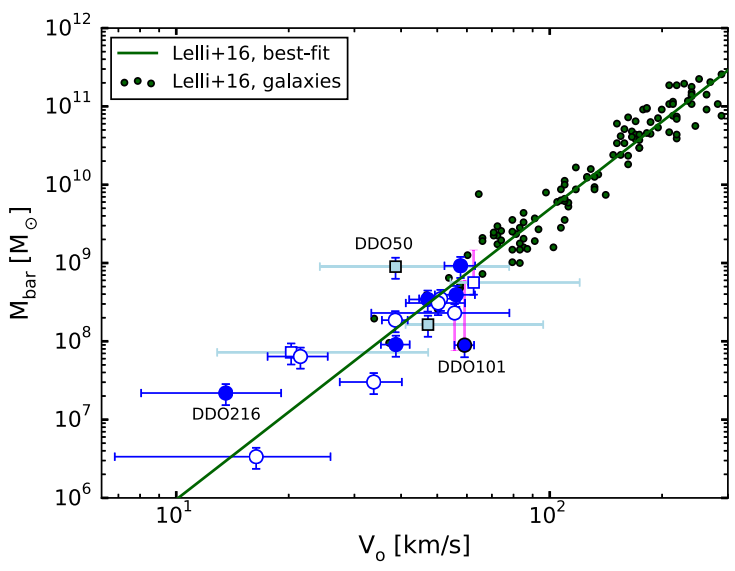

Figure 23. Same data as in Fig. 22 compared with the galaxies and the BTFR from Lelli et al. (2016).

more scattered, but this could owe entirely to the large error bars for these low-mass systems (that results from the increasingly important asymmetric-drift correction). Furthermore, there are few galaxies in this mass range and all of them have rising rotation curves (with the exception of DDO 216 that has rather peculiar kinematics; see Fig. 17, Section 5 and Appendix C).

\section{DISCUSSION}

\subsection{H I scaleheight}

All the galaxies in our sample have been analysed assuming a very thin $\mathrm{H}_{\mathrm{I}}$ disc with scaleheight $100 \mathrm{pc}$, independent of radius (see Section 3.2.1). This assumption would be fully justified in the case of spirals (e.g. Brinks \& Burton 1984; Olling 1996), but in the shallow potential of the dIrrs the gaseous layer can be quite thick especially in the outer regions of the disc (see e.g. O'Brien, Freeman \& van der Kruit 2010; Roychowdhury et al. 2010). In this section, we discuss how the presence of a thick disc could bias the results of our analysis.

In the presence of a thin disc, the observed $\mathrm{H}$ I emission can be easily related to the intrinsic properties of the galaxy: the observed velocity is a measure of gas rotation in the equatorial plane, the observed velocity dispersion is an unbiased measure of the chaotic motion of the gas and the intrinsic profile of the H I surface density can be obtained by simply correcting the observed profile for the $i$ of the galaxy (equation 4). In the presence of thick gaseous layers, the line of sight intercepts the emission coming from rings at different radial and vertical positions. As a consequence, the parameters obtained by assuming zero thickness may not be a precise measure of the kinematics of the galaxy.

2D methods as in O15 work on integrated maps (velocity fields) and they cannot take into account the presence of a thick disc. 3DB is more promising since the scaleheight is one of the parameters needed in the data cube fitting. We performed several tests with $3 \mathrm{DB}$, but we found that the fit is essentially insensitive of the $\mathrm{HI}$ thickness for small-medium values of the scaleheight $\left(z_{\mathrm{d}} \lesssim 0.7 \mathrm{kpc}\right)$ and returns unacceptable results for very thick $\mathrm{H}_{\mathrm{I}}$ discs. The reason for this is that $3 \mathrm{DB}$ fits one single ring at the time and it cannot include the emission coming from the extended vertical layers of the other rings.

We quantified the magnitude and the type of errors introduced by the assumption of thin disc as follows. We performed several tests on mock data cubes made with the task GALMOD (Sicking 1997) of the software package GIPSY (van der Hulst et al. 1992). We found that the assumption of a thin disc biases the results as follows:

(i) the surface-density profile tends to be shallower than the real profile;

(ii) the measured broadening of the $\mathrm{H}$ l line profiles is larger than the intrinsic velocity dispersion of the gas ( $\sigma_{v}$ is overestimated);

(iii) the representative velocity estimated from the $\mathrm{H}_{\mathrm{I}}$ line profiles may not trace the gas rotation velocity at certain locations.

Obviously, these effects influence the estimate of the final rotation curve: due to (iii) the observed velocities are no longer tracing the rotation on the equatorial plane, while (i) and (ii) bias the calculation of the asymmetric-drift correction (see Section 4.3.1). We found that the combination of these effects causes the circular velocities, obtained with both $2 \mathrm{D}$ and $3 \mathrm{D}$ methods assuming a thin disc, to be lower than the intrinsic velocities at smaller radii (similar to the beam smearing) and higher in the outermost disc. The magnitude of these differences depends mainly on the thickness of the H I discs, but the $i$ of the disc and the shape of the rotation curve are also important parameters. The biases described above are negligible for nearly face-on galaxies $\left(i<50^{\circ}\right)$, while a rising rotation curve, typical of dIrrs, amplifies the errors.

The thickness of the H I disc has never been incorporated in the derivation of the rotational velocity. In Iorio et al. (in preparation), we will present an original method to estimate in a self-consistent way the intrinsic kinematic properties of the galaxies taking into account the thickness of the $\mathrm{H}$ I disc under the assumption of vertical hydrostatic equilibrium. Applying this method to WLM (Fig. 21), we derived a scaleheight that is about $150 \mathrm{pc}$ in the centre and flares linearly with radius up to $600 \mathrm{pc}$, well above the $100 \mathrm{pc}$ assumed in our analysis. We compared the results obtained taking the $\mathrm{H}_{\mathrm{I}}$ thickness into account with the results obtained in the current work as described in Section 4: in the thick-disc model the peak of the profile of intrinsic surface density is higher by about $2 \mathrm{M}_{\odot} \mathrm{pc}^{-2}$, the velocity dispersion is lower by an average of $0.5 \mathrm{~km} \mathrm{~s}^{-1}$ and the rotation velocities have a maximum difference of about $4 \mathrm{~km} \mathrm{~s}^{-1}$. All these differences are compatible within the errors and we can conclude that our results for WLM are not seriously biased by the assumption of a thin disc.

As the scaleheight of a $\mathrm{HI}$ disc can be related to the velocity dispersion of the gas and the total volumetric density in the plane 

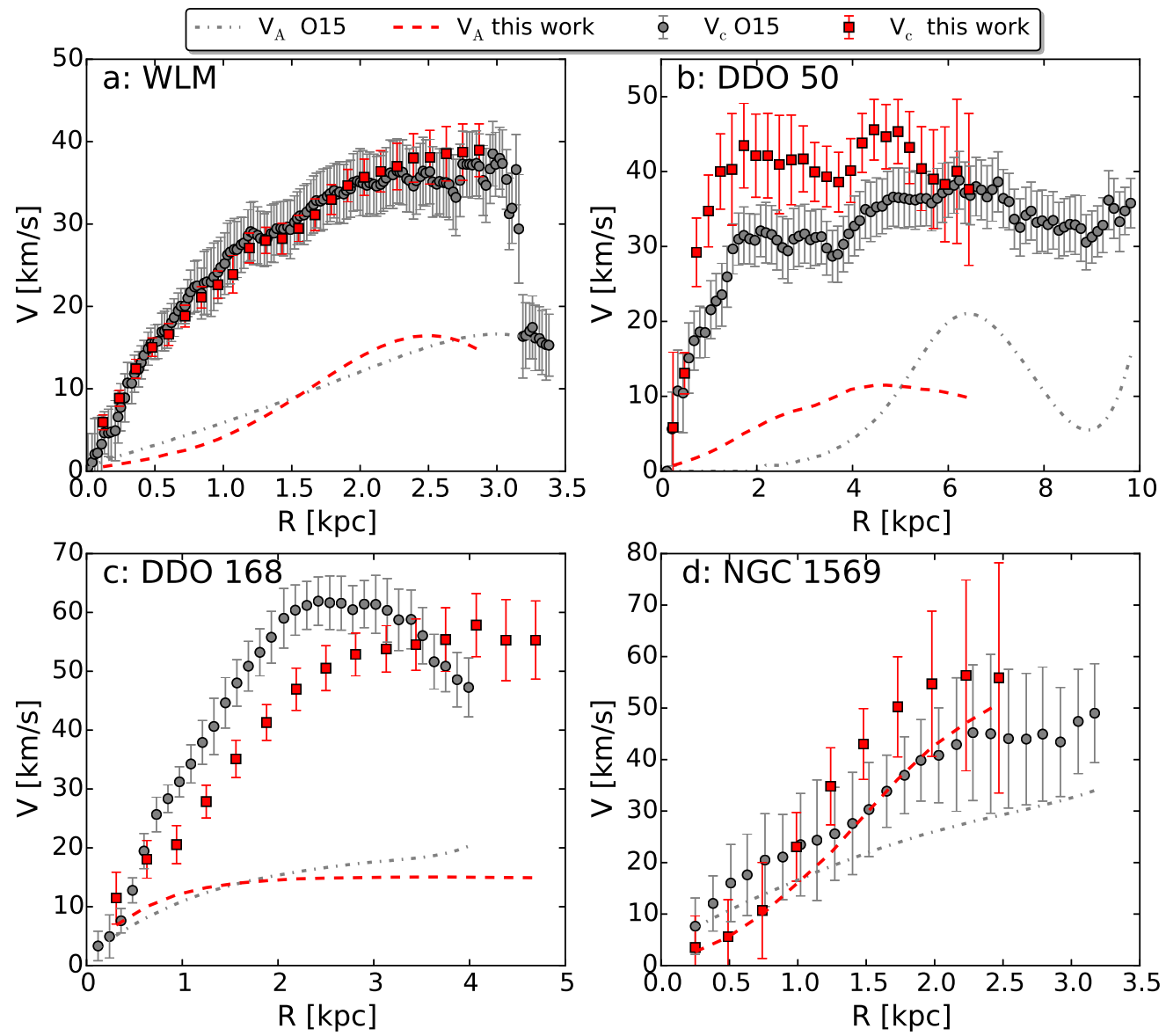

Figure 24. Circular velocity $\left(V_{\mathrm{c}}\right)$ and asymmetric-drift term $\left(V_{\mathrm{A}}\right)$ for four representative galaxies in our sample (a: WLM, b: DDO50, c: DDO 168, d: NGC 1569). The red squares and the red curves show the results found in this work, while the grey circles and the grey curves show the results obtained in O15.

of the galaxy as $z_{\mathrm{d}} \propto \sigma_{v} \rho^{-0.5}$ (Olling 1995; Ott et al. 2001), we can define an average density in terms of circular velocity so that $\mathrm{Z}_{\mathrm{d}} \mathrm{R}^{-1} \propto \sigma_{\mathrm{v}} \mathrm{V}_{\mathrm{c}}^{-1}$. Therefore, WLM is expected to be representative of our sample of galaxies, in term of disc thickness (see Table 2): the bias due to the $\mathrm{H}_{\mathrm{I}}$ thickness is likely to be significantly larger only for DDO 210, DDO 216 and NGC 1569. However, in these galaxies the rotation curve is already very uncertain and the quoted errors are so large that should be still inclusive of the errors due to the thin-disc assumption. In conclusion, we are confident that all the rotation curves and velocity dispersions found in this work are not seriously biased by the assumption that the $\mathrm{H}$ I disc is thin.

\subsection{Comparison to the standard 2D approach}

In this section, we compare the rotation curves obtained with our method with the ones obtained in $\mathrm{O} 15$ for the same data set with the classical 2D approach. As a general feature, the rotation curves of O15 reach larger radii: the median difference on the radial extension is about $700 \mathrm{pc}$ and the galaxies with the largest discrepancies are: DDO 50 (3.4 kpc), DDO 87 (2.2 kpc) and NGC 2366 (1.8 kpc). DDO 216 has rotation curves of similar extensions, while our rotation curves for DDO 210 and DDO 168 are more extended by about 200 and $700 \mathrm{pc}$, respectively. These differences are due to the different approach in the choices on the outermost radii considered in the kinematic fit. We decided to include only the part of the galaxy with clear information on the rotation motion and not dominated by the noise (see Section 4.1.2), as shown by the elliptical rings over plotted to the H I maps and $\mathrm{H}$ i velocity fields (Box C in Figs 5-21). In contrast, $\mathrm{O} 15$ extended the fit as much as possible, but in some cases the outer part of the rotation curves might be extracted mainly from emission around the minor axis that have poor information on the rotational motion. The peril of this approach is that the rotation curves at large radii can be quite unreliable or show artefacts as for example in WLM (Fig. 24a) where the rotation curve of O15 shows an abrupt drop-off after the outermost radii used in our analysis.

Concerning the shapes of rotation curves, about half of our results are compatible with $\mathrm{O} 15$ within the errors. When differences are present they can be due to a combination of three different reasons: (i) an intrinsic difference in the best-fitting projected rotational velocities $\left(V_{\mathrm{rot}} \sin i\right)$, (ii) a difference in the best-fitting $i$, PA or centre and (iii) a difference in the asymmetric-drift correction terms $\left(V_{\mathrm{A}}\right)$. Most of the cases are due to (ii) and (iii); only one galaxy (DDO 168) seems to have a significant discrepancy caused by the two different fitting methods. This galaxy is shown in Fig. 24(c), while examples of (ii) and (iii) are shown in Figs 24(b) and (d). The galaxies showing the largest discrepancies are listed below.

(1) CVnidwa. Our final rotation curve is systematically higher than that of $\mathrm{O} 15$ by about $5 \mathrm{~km} \mathrm{~s}^{-1}$. This difference is totally dominated by the discrepancy in the asymmetric-drift correction term. Our $V_{\mathrm{A}}$ is already significant at the inner radii, while the $V_{\mathrm{A}}$ of $\mathrm{O} 15$ is negligible out to $2 \mathrm{kpc}$. These differences are ascribed to the different radial trend of the intrinsic surface density profile caused by discrepancies in the best-fitting PA. 
(2) DDO 47. The circular velocity shows a discrepancy of about $7 \mathrm{~km} \mathrm{~s}^{-1}$ in the inner part of the disc $(R<2 \mathrm{kpc})$ due to a discrepancy on the best-fitting $i$ : 015 used $i \approx 55^{\circ}$ while we used $i=37^{\circ}$ independent of radius.

(3) DDO 50. Discrepancies of about $10 \mathrm{~km} \mathrm{~s}^{-1}$ (see Fig. 24b) are due to the different assumption on the value of $i$ (about $50^{\circ}$ in $\mathrm{O} 15$ and $33^{\circ}$ in this work). Our curve is also less extended (see Fig. 7).

(4) DDO 52. The final rotation curve of O15 is systemically higher due to the different values of $i$ (about $37^{\circ}$ in 015 and $55^{\circ}$ in this work).

(5) DDO 87. Our final rotation curve shows a steeper rising due to a discrepancy in the assumed $i$ : we used $i=40^{\circ}$ independent of radius, while in $\mathrm{O} 15 i$ varies between $60^{\circ}$ and $40^{\circ}$.

(6) DDO 168. The final rotation curves are quite different (see Fig. 24c). In O15, the curve flattens at about $2 \mathrm{kpc}$ and starts to decrease at $3 \mathrm{kpc}$, while our rotation curve shows a less steep rising in the inner part and a flattening at about $3.5 \mathrm{kpc}$. These discrepancies are due mainly to an intrinsic difference in the best-fitting projected rotation curve found with the two methods. The differences are further increased by the different assumptions on $i\left(i \approx 60^{\circ}\right.$ in this work, $i \approx 40^{\circ}$ in $\mathrm{O} 15$ ).

(7) NGC 1569. The circular rotation curve found in this work rises steeper with respect to the rotation curve reported in $\mathrm{O} 15$ (see Fig. 24d). The difference is caused by the asymmetric-drift correction given that with our 3D method we find a different radial trend of both the velocity dispersion and the intrinsic surface density.

(8) NGC 2366. Our final rotation curve is systematically lower in the inner disc $(R<2 \mathrm{kpc})$. The cause is a combination of a different position of the galactic centre and different PA. We used a constant $\mathrm{PA}$ of about $40^{\circ}$, while in $\mathrm{O} 15$ the PA grows from $20^{\circ}$ to $40^{\circ}$ in the first two kpc.

\section{SUMMARY}

We presented a study of the kinematics of the $\mathrm{HI}$ discs for 17 dIrrs taken from the public survey LITTLE THINGS (Hunter et al. 2012). The main goal of this work is to make available to the community a sample of high-quality rotation curves of dIrrs ready-to-use to perform dynamical studies. The tabulated quantities $\left(V_{\mathrm{rot}}, V_{\mathrm{c}}, V_{\mathrm{A}}\right.$, $\left.\sigma_{v}\right)$ and $\mathrm{H}_{\mathrm{I}}$ surface-density profile $\left(\Sigma_{\text {int }}\right)$ are available in the online version of the paper. The key points of this work are listed below.

(1) We derived the rotation curves from the $\mathrm{H}_{\mathrm{I}}$ data cubes with a state-of-art technique: the publicly available software ${ }^{3 \mathrm{D}}$ BAROLO (Di Teodoro \& Fraternali 2015). It fits 3D models to the data cubes without explicitly extracting velocity fields. The fit in the $3 \mathrm{D}$ space of the data cubes ensures full control of the observational effects and in particular a proper account of beam smearing.

(2) The maximum radii used in the fits have been chosen with great care from the $\mathrm{H}_{\mathrm{I}}$ total map. This ensures a robust estimate of the kinematics avoiding regions of the galaxy dominated by the noise and with scant information about the gas rotation.

(3) We developed a method to take into account the uncertainties of the asymmetric-drift correction. As a consequence, the quoted errors on the rotation curves are representative of the real uncertainties. The inclusion of these errors is fundamental in galaxies for which the calculation of the circular velocity is highly dominated by the asymmetric-drift correction (e.g. DDO 210).

(4) We estimated how our results can be biased by the assumption of a thin H I disc. We analysed in detail the galaxy WLM and we found that the $\mathrm{H}_{\mathrm{I}}$ layer flares linearly from about 150 to $600 \mathrm{pc}$, well above the $100 \mathrm{pc}$ assumed in our analysis. The presence of such thick disc introduces systematic errors on the estimate of the velocity dispersion, intrinsic $\mathrm{H}_{\mathrm{I}}$ surface density and circular velocity. However, the differences are compatible within the quoted uncertainties. The thickness of WLM should be representative of the galaxies of our sample, so we can conclude that our results are not seriously biased by the assumption of thin disc.

(5) The rotation curves obtained in this work have been used to test the BTFR in the low-mass regime $\left(10^{6} \lesssim \mathrm{M}_{\text {bar }} / \mathrm{M}_{\odot} \lesssim 10^{9}\right)$. We found that our results are compatible in slope, normalization and scatter with the work of Lelli et al. (2016) at higher mass $\left(\mathrm{M}_{\mathrm{bar}}>10^{8} \mathrm{M}_{\odot}\right)$.

\section{ACKNOWLEDGEMENTS}

This research made use of the 'Local Irregulars That Trace Luminosity Extremes, The H I Nearby Galaxy Survey' (LITTLE THINGS)' data sample. We would like to thank the referee Mark Wilkinson and Antonino Marasco for useful comments that improved this manuscript, and Se-Heon Oh and Federico Lelli for kindly making their data available. GB acknowledges financial support by the Spanish Ministry of Economy and Competitiveness (MINECO) under the Ramon y Cajal Programme (RYC-2012-11537). JIR would like to acknowledge support from STFC consolidated grant ST/M000990/1 and the MERAC foundation. The research has made use of the NASA/IPAC Extragalactic Database (NED) that is operated by the Jet Propulsion Laboratory, California Institute of Technology, under contract with the National Aeronautics and Space Administration.

\section{REFERENCES}

Baillard A. et al., 2011, A\&A, 532, A74

Battaglia G., Fraternali F., Oosterloo T., Sancisi R., 2006, A\&A, 447, 49

Begeman K. G., 1987, PhD thesis, Kapteyn Institute

Begum A., Chengalur J. N., Karachentsev I. D., Sharina M. E., 2008, MNRAS, 386, 138

Bosma A., 1978, PhD thesis, Groningen Univ.

Bosma A., 1981, AJ, 86, 1791

Bosma A., Goss W. M., Allen R. J., 1981, A\&A, 93, 106

Boylan-Kolchin M., Bullock J. S., Kaplinghat M., 2011, MNRAS, 415, L40

Brinks E., Burton W. B., 1984, A\&A, 141, 195

Brook C. B., Santos-Santos I., Stinson G., 2016, MNRAS, 459, 638

Bureau M., Carignan C., 2002, AJ, 123, 1316

Carignan C., Purton C., 1998, ApJ, 506, 125

Casertano S., van Gorkom J. H., 1991, AJ, 101, 1231

Cole A. A. et al., 1999, AJ, 118, 1657

Cook D. O. et al., 2014, MNRAS, 445, 881

Côté S., Carignan C., Freeman K. C., 2000, AJ, 120, 3027

Croxall K. V., van Zee L., Lee H., Skillman E. D., Lee J. C., Côté S., Kennicutt R. C., Jr, Miller B. W., 2009, ApJ, 705, 723

de Blok W. J. G., Bosma A., 2002, A\&A, 385, 816

de Blok W. J. G., Walter F., Brinks E., Trachternach C., Oh S.-H., Kennicutt R. C., Jr, 2008, AJ, 136, 2648

Di Cintio A., Lelli F., 2016, MNRAS, 456, L127

Di Teodoro E. M., Fraternali F., 2015, MNRAS, 451, 3021

Fraternali F., Oosterloo T., Sancisi R., van Moorsel G., 2001, ApJ, 562, L47

Fraternali F., van Moorsel G., Sancisi R., Oosterloo T., 2002, AJ, 123, 3124

García-Ruiz I., Sancisi R., Kuijken K., 2002, A\&A, 394, 769

Gentile G., Salucci P., Klein U., Vergani D., Kalberla P., 2004, MNRAS, 351,903

Gentile G., Burkert A., Salucci P., Klein U., Walter F., 2005, ApJ, 634, L145

Georgiev T. B., Karachentsev I. D., Tikhonov N. A., 1997, Astron. Lett., 23, 514

Hunter D. A., Elmegreen B. G., 2006, ApJS, 162, 49 
Hunter D. A., Elmegreen B. G., van Woerden H., 2001, ApJ, 556, 773

Hunter D. A., Elmegreen B. G., Oh S.-H., Anderson E., Nordgren T. E., Massey P., Wilsey N., Riabokin M., 2011, AJ, 142, 121

Hunter D. A. et al., 2012, AJ, 144, 134

Jarrett T. H., Chester T., Cutri R., Schneider S. E., Huchra J. P., 2003, AJ, 125,525

Johnson M., Hunter D. A., Oh S.-H., Zhang H.-X., Elmegreen B., Brinks E., Tollerud E., Herrmann K., 2012, AJ, 144, 152

Józsa G. I. G., Kenn F., Klein U., Oosterloo T. A., 2007, A\&A, 468, 731

Kamphuis P., Józsa G. I. G., Oh S.-H., Spekkens K., Urbancic N., Serra P., Koribalski B. S., Dettmar R.-J., 2015, MNRAS, 452, 3139

Karachentsev I. D., Makarov D. I., Kaisina E. I., 2013, AJ, 145, 101

Kirby E. N., Bullock J. S., Boylan-Kolchin M., Kaplinghat M., Cohen J. G., 2014, MNRAS, 439, 1015

Klypin A., Kravtsov A. V., Valenzuela O., Prada F., 1999, ApJ, 522, 82

Knapen J. H., Erroz-Ferrer S., Roa J., Bakos J., Cisternas M., Leaman R., Szymanek N., 2014, A\&A, 569, A91

Krajnović D., Cappellari M., de Zeeuw P. T., Copin Y., 2006, MNRAS, 366, 787

Leaman R. et al., 2012, ApJ, 750, 33

Lelli F., Fraternali F., Sancisi R., 2010, A\&A, 516, A11

Lelli F., Verheijen M., Fraternali F., Sancisi R., 2012a, A\&A, 537, A72

Lelli F., Verheijen M., Fraternali F., Sancisi R., 2012b, A\&A, 544, A145

Lelli F., Verheijen M., Fraternali F., 2014a, MNRAS, 445, 1694

Lelli F., Verheijen M., Fraternali F., 2014b, A\&A, 566, A71

Lelli F., McGaugh S. S., Schombert J. M., 2016, ApJ, 816, L14

McConnachie A. W., 2012, AJ, 144, 4

McGaugh S. S., 2012, AJ, 143, 40

McGaugh S. S., Schombert J. M., Bothun G. D., de Blok W. J. G., 2000, ApJ, 533, L99

Makarova L., 1999, A\&AS, 139, 491

O'Brien J. C., Freeman K. C., van der Kruit P. C., 2010, A\&A, 515, A62

Oh S.-H., de Blok W. J. G., Walter F., Brinks E., Kennicutt R. C., Jr, 2008, AJ, 136, 2761

Oh S.-H., Brook C., Governato F., Brinks E., Mayer L., de Blok W. J. G., Brooks A., Walter F., 2011, AJ, 142, 24

Oh S.-H. et al., 2015, AJ, 149, 180 (O15)

Olling R. P., 1995, AJ, 110, 591

Olling R. P., 1996, AJ, 112, 481

Oman K. A., Navarro J. F., Sales L. V., Fattahi A., Frenk C. S., Sawala T., Schaller M., White S. D. M., 2016, MNRAS, 460, 3610

Ott J., Walter F., Brinks E., Van Dyk S. D., Dirsch B., Klein U., 2001, AJ, 122,3070

Puche D., Westpfahl D., Brinks E., Roy J.-R., 1992, AJ, 103, 1841

Read J. I., Wilkinson M. I., Evans N. W., Gilmore G., Kleyna J. T., 2006, MNRAS, 367, 387

Read J. I., Iorio G., Agertz O., Fraternali F., 2016a, preprint (arXiv:1607.03127)

Read J. I., Agertz O., Collins M. L. M., 2016b, MNRAS, 459, 2573

Read J. I., Iorio G., Agertz O., Fraternali F., 2016c, MNRAS, 462, 3628

Roberts M. S., 1975, Radio Observations of Neutral Hydrogen in Galaxies. Univ. Chicago Press, Chicago, p. 309

Rogstad D. H., Shostak G. S., 1971, A\&A, 13, 99

Rogstad D. H., Lockhart I. A., Wright M. C. H., 1974, ApJ, 193, 309

Roychowdhury S., Chengalur J. N., Begum A., Karachentsev I. D., 2010, MNRAS, 404, L60

Schoenmakers R. H. M., Franx M., de Zeeuw P. T., 1997, MNRAS, 292, 349

Sicking F. J., 1997, PhD thesis, Univ. Groningen

Spekkens K., Sellwood J. A., 2007, ApJ, 664, 204

Stil J. M., Israel F. P., 2002a, A\&A, 389, 42

Stil J. M., Israel F. P., 2002b, A\&A, 392, 473

Swaters R. A., 1999, PhD thesis, Rijksuniversiteit Groningen

Swaters R. A., Schoenmakers R. H. M., Sancisi R., van Albada T. S., 1999, MNRAS, 304, 330

Tamburro D., Rix H.-W., Leroy A. K., Mac Low M.-M., Walter F., Kennicutt R. C., Brinks E., de Blok W. J. G., 2009, AJ, 137, 4424

Taylor C. L., Kobulnicky H. A., Skillman E. D., 1998, AJ, 116, 2746 van Albada T. S., Bahcall J. N., Begeman K., Sancisi R., 1985, ApJ, 295, 305

van der Hulst J. M., Terlouw J. P., Begeman K. G., Zwitser W., Roelfsema P. R., 1992, in Worrall D. M., Biemesderfer C., Barnes J., eds, ASP Conf. Ser. Vol. 25, Astronomical Data Analysis Software and Systems I. Astron. Soc. Pac., San Francisco, p. 131

van Eymeren J., Marcelin M., Koribalski B., Dettmar R.-J., Bomans D. J., Gach J.-L., Balard P., 2009a, A\&A, 493, 511

van Eymeren J., Marcelin M., Koribalski B. S., Dettmar R.-J., Bomans D. J., Gach J.-L., Balard P., 2009b, A\&A, 505, 105

Verheijen M. A. W., 2001, ApJ, 563, 694

Verheijen M. A. W., Sancisi R., 2001, A\&A, 370, 765

Walter F., Brinks E., 2001, AJ, 121, 3026

Whiting M. T., 2012, MNRAS, 421, 3242

Zhang H.-X., Hunter D. A., Elmegreen B. G., Gao Y., Schruba A., 2012, AJ, 143, 47

\section{SUPPORTING INFORMATION}

Supplementary data are available at MNRAS online.

\section{BTFR_data.txt results.zip}

Please note: Oxford University Press is not responsible for the content or functionality of any supporting materials supplied by the authors. Any queries (other than missing material) should be directed to the corresponding author for the article.

\section{APPENDIX A: ESTIMATE OF THE GEOMETRICAL PARAMETERS}

It is important to initialize the fit of the $\mathrm{H}_{\mathrm{I}}$ data cubes with good estimates of the geometrical parameters: centre, $i$ and PA of the $\mathrm{H}_{\mathrm{I}}$ disc (see Section 4.2). We used three different methods:

(i) $3 \mathrm{DB}: 3 \mathrm{DB}$ can automatically estimate the geometrical parameters analysing the zeroth moment ( $\mathrm{H}_{\mathrm{I}}$ total map) and the first moment (velocity field) of the data cube. The $i$ is estimated by fitting $\mathrm{H}_{\mathrm{I}}$ model maps to the observed $\mathrm{H}_{\mathrm{I}}$ total map, while the PA is calculated as the orientation of the line that maximizes the velocity gradient in the velocity field. Finally, the centre is estimated as the flux-weighted average position of the source. 3DB builds the total map and the velocity field using a source-finding algorithm (DUCHAMP, Whiting 2012), separating the source pixels from the noise pixels on the basis of a $\mathrm{S} / \mathrm{N}$ threshold. As a consequence, the final maps and the estimate of the disc parameters depend on the choice of this threshold. For this reason, we repeat this analysis using $\mathrm{S} / \mathrm{N}$ threshold ranging from 2.5 to 5 . Finally, we average the values on the range of $\mathrm{S} / \mathrm{N}$ where the estimated parameters are almost constant.

(ii) RingFit: We developed a simple script, called RingFit, to fit ellipses to the iso-density contours at different radii of our total $\mathrm{HI}$ maps (Section 4.1.1). From the properties of the fitted ellipses, we can estimate the geometrical parameters of the $\mathrm{H}$ I disc at different radii.

(iii) Isophotal fitting of the optical maps: The geometrical parameters are derived from the $V$-band photometry by Hunter \& Elmegreen (2006).

Figs A1 and A2 show two examples of the estimate of $i$ with the three different methods. In the first case (DDO 52) the methods are in good agreement, while in the second case (DDO 210) the results are not compatible (notice that in this case the $\mathrm{H}$ I contours deviate strongly from elliptical shape, see Box C in Fig. 16). Usually, the 


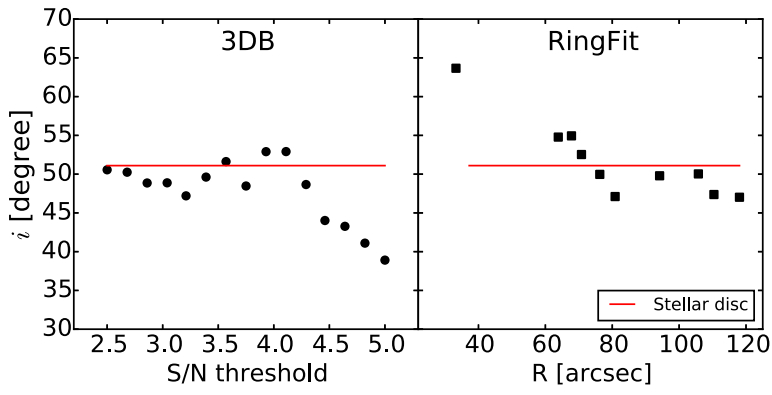

Figure A1. Initial estimate of the inclination angle for DDO 52 with 3DB (left-hand panel) and with the fit of the iso-density contours of the $\mathrm{H}$ I total map (right-hand panel). The red lines show the inclination of the optical disc. See the text for details.

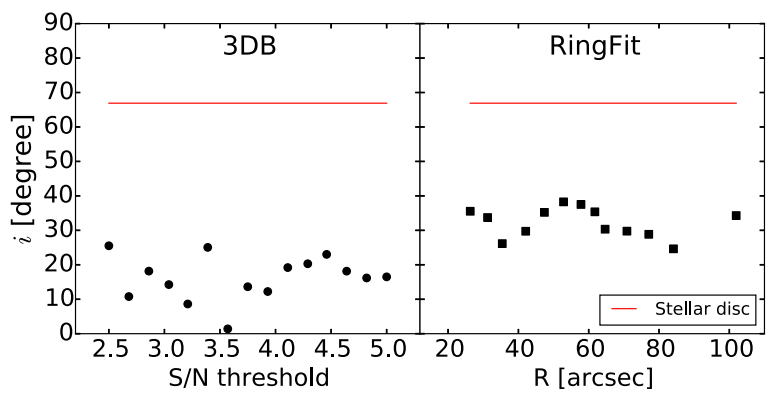

Figure A2. Same as Fig. A1 but for DDO 210.

optical disc has a more regular morphology with respect to the $\mathrm{H}$ I disc; therefore, we give more weight to the geometrical parameters estimated from Hunter \& Elmegreen (2006). When the geometry of the stellar and that of the $\mathrm{H}$ I disc do not show a clear agreement (Fig. A2) or the stellar disc presents peculiar features such as strong bars, we use the other two methods. More details can be found in the description of the individual galaxies in Section 5.

\section{APPENDIX B: PLOT LAYOUT}

In this appendix, we report the caption that describes Figs 5-21.

(i) Box A: Rotation velocity estimated with $3 \mathrm{DB}$ ( $V_{\text {rot }}$, blue line), asymmetric-drift correction ( $V_{\mathrm{A}}$, grey circles) and final corrected circular velocity $\left(V_{\mathrm{c}}\right.$, black circles).

(ii) Box B:

Top: Inclination (left-hand panel) and position angle (right-hand panel) found with $3 \mathrm{DB}$ as functions of radius $R$. The red lines show the fit used to obtain the rotation velocity (see Section 4.2), while the black dashed line indicates the median of the data. Bottom: H I velocity dispersion estimated with 3DB (left-hand panel) and intrinsic surface density of the Hi disc not corrected for Helium (right-hand panel) as function of radius $R$. In the lefthand panel, the black dashed line indicates the median of the data. The empty circles (if present) represent the points excluded both from the calculation of the asymmetric-drift correction (see Section 4.3.1) and from the calculation of the median. The excluded points belong to rings where the velocity dispersion found with $3 \mathrm{DB}$ is discrepant or peculiar with respect to the global trend (see Section 4.3.4).

(iii) Box C:

Left-hand panel: Stellar emission overlaid with the iso-density contours of the total $\mathrm{H}_{\mathrm{I}}$ emission. The thick contour indicates $\sigma_{3 \mathrm{~T}}$, the
$3 \sigma$ pseudo-noise of the total map (see Section 4.1.2 and Table 1). Right-hand panel: Velocity field obtained as the first moment of the data cube. The thick contour highlights the systemic velocity (see Table 2). A physical scale is plotted on the bottom right corner of the panel, while the beam of the H I observation is shown in the bottom left corner.

The crosses and the ellipses show the assumed galactic centre and the last used ring, respectively (see Table 2).

(iv) Box D: PV diagram along the major (left-hand panel) and the minor (right-hand panel) axes of the H I disc. The black and the red contours show respectively the iso-density contours of the galaxy and the best-fitting model found with 3DB. The horizontal black dashed lines indicate the systemic velocity.

\section{APPENDIX C: AN ALTERNATIVE SCENARIO FOR DDO 216}

DDO 216 shows a peculiar kinematics: a velocity gradient of about $10 \mathrm{~km} \mathrm{~s}^{-1}$ appears clearly in the velocity field (right-hand panel in Box C in Fig. 17) and in the channel maps, but it could be entirely due to a single 'cloud' at a discrepant velocity in the approaching side of the galaxy (Stil \& Israel 2002a). The analysis of the channel maps partially supports this hypothesis: the receding and the approaching sides of the galaxy seem to have a slightly distinct kinematics and the emission of the two halves overlaps between -186 and $-190 \mathrm{~km} \mathrm{~s}^{-1}$, while the $V_{\text {sys }}$ found with 3DB is around $-180 \mathrm{~km} \mathrm{~s}^{-1}$. At the same time, the total H I map does not show the clear presence of a separate component at the supposed position of the cloud (left-hand panel in Box C in Fig. 17). In conclusion, we did not have sufficient elements to confirm or exclude the existence of a Hi component other than the disc of DDO 216. We can summarize the two hypotheses as:

(i) scenario1: The main velocity gradient is genuine and totally due to the rotation of the $\mathrm{H}$ I disc.

(ii) scenario2: The main velocity gradient is due to an $\mathrm{H}_{\mathrm{I}}$ substructure with a systemic velocity that differs by about $20 \mathrm{~km} \mathrm{~s}^{-1}$ from that of the $\mathrm{H}$ I disc.

The scenario1 is analysed in Section 5 while in this appendix we describe the analysis of the scenario2. First of all, we masked the region of the data cube containing the emission of the supposed $\mathrm{H}$ I cloud. The mask has been built by inspecting the channel maps using the task PYBLOT of GIPSY. Then we performed the kinematic fit of the 'clean' data cube with $3 \mathrm{DB}$. We set $i_{\text {ini }}, \mathrm{PA}_{\text {ini }}$ and the galactic centre to the values obtained from the stellar disc (Hunter $\&$ Elmegreen 2006). Contrarily to the scenario1 (Section 5), we used the $V_{\text {sys }}$ estimated with $3 \mathrm{DB}$, the two estimates differ by about $8 \mathrm{~km} \mathrm{~s}^{-1}$, while the final best-fitting PA $\left(120^{\circ}\right)$ is tilted of about $10^{\circ}-15^{\circ}$ with respect to the values found in the scenario1 (Box B in Fig. 17).

Without the 'cloud' the velocity gradient almost disappears and the kinematics is dominated by the chaotic motion of the gas as it is clearly visible in the PVs in Fig. C1. As a consequence, the final rotation curve is 'shaped' by the asymmetric-drift correction. Fig. C2 compares the rotation curve found for the scenario1 and for the scenario2: the velocities are compatible within the errors, but in scenariol $V_{\mathrm{c}}$ is systematically higher by about $4 \mathrm{~km} \mathrm{~s}^{-1}$ and it extends to larger radii.

Kirby et al. (2014) found that the stellar component of DDO 216 is rotating in the same direction as the gas with a $V_{\text {rot }}$ of about $10 \mathrm{~km} \mathrm{~s}^{-1}$, perfectly compatible with scenario1 (see Fig. C2). This 


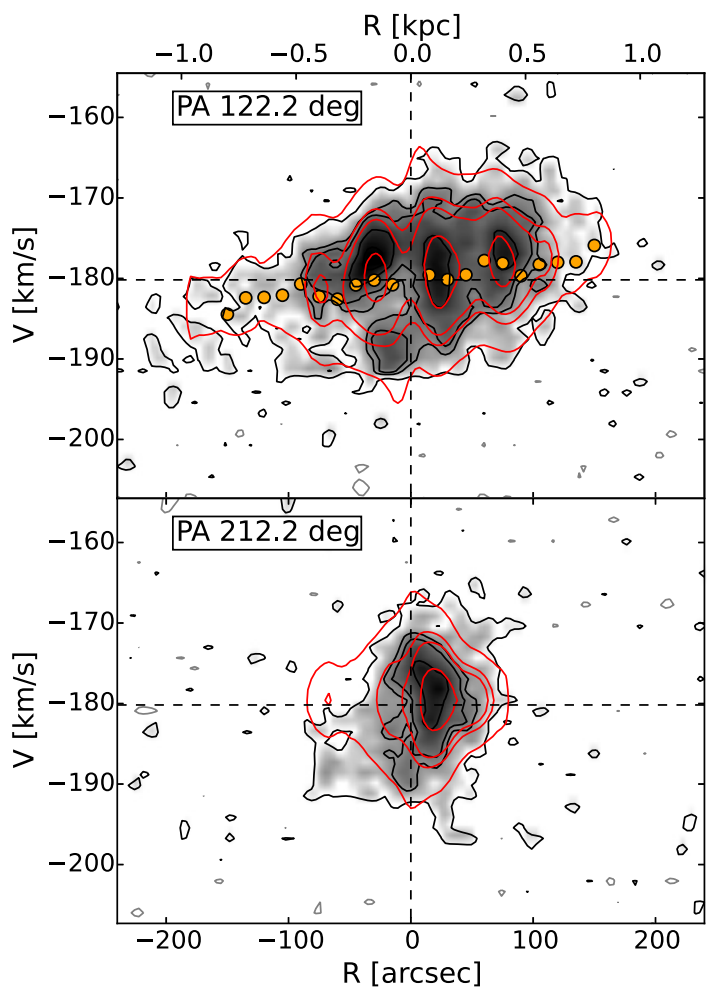

Figure C1. PV along the major axis (upper panel) and the minor axis (bottom panel) for the scenario2 of the galaxy DDO 216 (see text). The black contours indicate the data cube while the red contours show the best-fitting model found with 3DB. Contours at $(2+6 n) \sigma_{\mathrm{ch}}$, where $\sigma_{\mathrm{ch}}=0.91 \mathrm{mJy}$ $\mathrm{bm}^{-1}$, the grey contours are at $-2 \sigma_{\mathrm{ch}}$. The yellow points indicate the rotational velocity found with $3 \mathrm{DB}$.

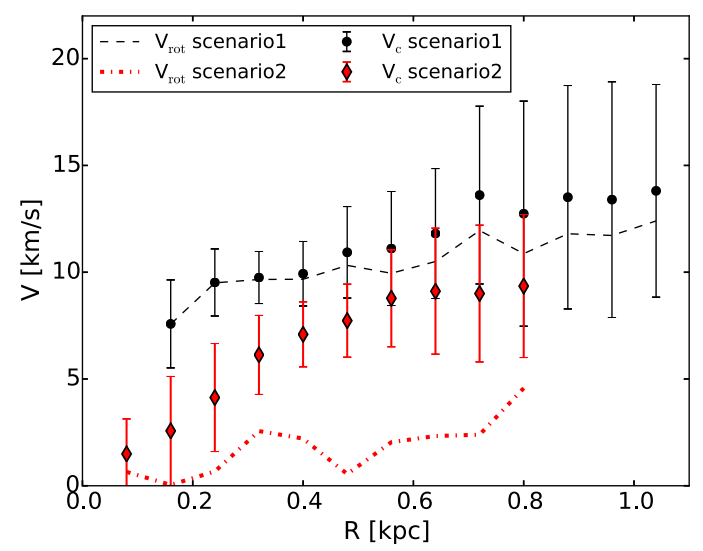

Figure C2. Rotational velocities (red dashed line) and circular velocities (red points) found for the scenario2 of DDO 216 (see text). The rotation curves of the scenario1 are also shown for comparison.

velocity gradient is observed also in red giant stars, so the rotation is genuine and it is not caused by short-term hydrodynamical events (e.g. bubbles), which influence the gas kinematics but not the kinematics of the old stellar populations. However, the centre of the rotation and $V_{\text {sys }}$ are quite different from what we found for scenario1 and they are instead compatible with scenario2. Further studies are needed to understand if the gas in this peculiar galaxy is rotating, which is crucial to accurately estimate its dynamical mass.

\section{APPENDIX D: TYPICAL SETUP FOR ${ }^{3 D_{B}}$ AROLO}

In this appendix, we report the keywords and their values for our typical parameter file passed to 3DB. For a detailed description of the keywords, see the documentation of $3 \mathrm{DB}^{5}$ ('...' means that the values depends on the analysed galaxy).

(i) FITSFILE...; name of the data cube to fit.

(ii) GALFIT TRUE; enable the 3D fitting of the data cube.

(iii) NRADII...; number of rings to be used in the fit.

(iv) RADSEP...; separation between rings in arcsec.

(v) VROT 30; initial guess for the rotation velocity in $\mathrm{km} \mathrm{s}^{-1}$.

(vi) VDISP 8; initial guess for the velocity dispersion in $\mathrm{km} \mathrm{s}^{-1}$.

(vii) INC...; initial guess for $i$ in degree ( $i_{\text {ini }}$, see Table 2 ).

(viii) PA...; initial guess for the PA in degree $\left(\mathrm{PA}_{\text {ini }}\right.$, see Table 2$)$.

(ix) VSYS...; initial guess for the systemic velocity in $\mathrm{km} \mathrm{s}^{-1}$.

(x) XPOS...; initial guess for the $X$ coordinate of the centre.

(xi) YPOS...; initial guess for the $Y$ coordinate of the centre.

(xii) $\mathrm{ZO} 100 / f_{\mathrm{c}}$; initial guess for the scaleheight in arcsec, $f_{\mathrm{c}}$ is the conversion factor from arcsec to pc and it is listed in Table 1 for each galaxy.

(xiii) LTYPE 1; use a Gaussian profile for the H I vertical distribution.

(xiv) FREE VROT VDISP INC PA; parameters to fit with 3DB.

(xv) FTYPE 2; use the absolute differences between models and data cube as residuals.

(xvi) WFUNC 1; use the cosine of the azimuthal angle as weigthing function for the fit (see Section 4.2).

(xvii) CDENS 10; this parameter sets (in a non-trivial way) the number of 'clouds' to be used in the building of the ring models.

(xviii) NV 200; number of sub-clouds in which each cloud (see above) is divided to populate the spectral axis of the ring models.

(xix) TOL 1E-3; tolerance condition to stop the fit.

(xx) MASK Smooth; create a mask for the fit smoothing the data cube.

(xxi) BLANKCUT 2.5; include in the mask (see above) only the pixels with $\mathrm{S} / \mathrm{N}>2.5$.

(xxii) SIDE B; fit the whole galaxy.

(xxiii) TWOSTAGE TRUE; enable the two-stage method described in Section 4.2.

(xxiv) BWEIGHT 1; set the weight of the blank pixels (see Di Teodoro \& Fraternali 2015 for details).

(xxv) DELTAINC 40; $i$ can assume values only between INCDELTAINC and INC+DELTAINC.

(xxvi) DELTAPA 80; same as above, but for the PA.

(xxvii) FLAGERRORS true; enable the estimate of the errors on the fitted parameters.

(xxviii) STARTRAD 1; avoid the fit of the first ring placed by default at RADSEP/4.

(xxix) LINEAR 0.424; instrumental resolution ( $\left.\sigma_{\text {inst }}\right)$ in unity of channel width. In all our galaxies, the instrumental FWHM is equal to the channel separation, so $\sigma_{\text {inst }}=1 / 2.355=0.424$.

If the parameters XPOS, YPOS, VSYS, PA, INC are omitted, 3DB estimates them as described in Appendix A.

\footnotetext{
${ }^{5}$ http://editeodoro.github.io/Bbarolo/documentation/
}

This paper has been typeset from a $\mathrm{T}_{\mathrm{E}} \mathrm{X} / \mathrm{L} \mathrm{T}_{\mathrm{E}} \mathrm{X}$ file prepared by the author. 